\title{
Guidelines for Biodiversity Assessment and Monitoring Por Protected Areas
}

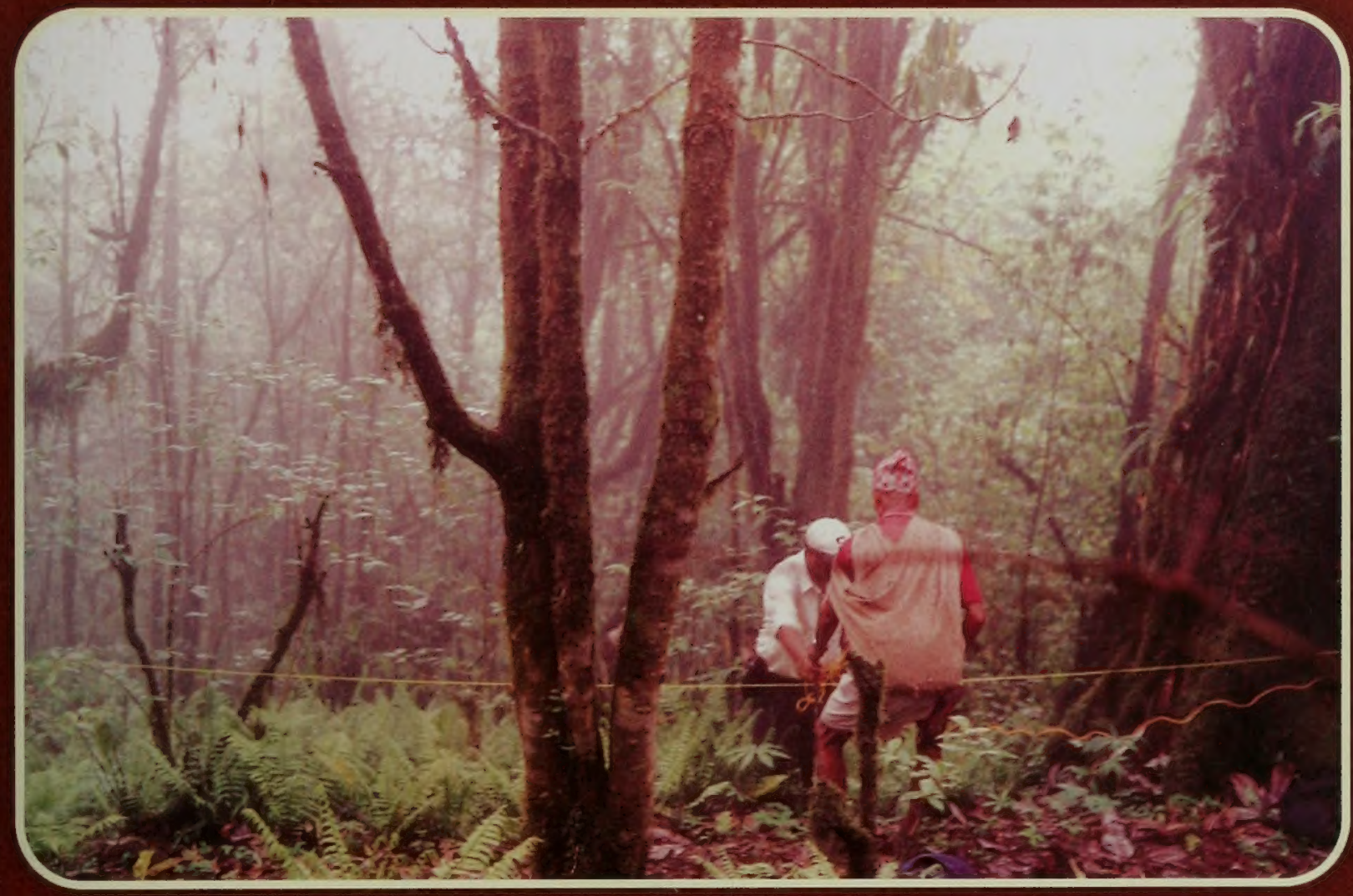

\section{Authors}

Graham Tucker (Ecological Solutions), Philip Bubb (UNEP-WCMC), Mireille de Heer (UNEP-WCMC), Lera Miles (UNEP-WCMC), Anna Lawrence and Jeanette van Rijsoort

(Oxford University Environmental Change Institute), Siddhartha B. Bajracharya (KMTNC), Ram Chandra Nepal (KMTNC), Roshan Sherchan (KMTNC) and Nawaraj Chapagain (KMTNC).

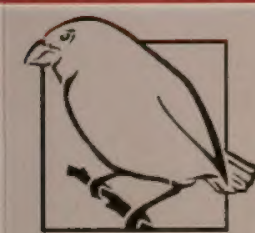

DARWIN INITIATIVE

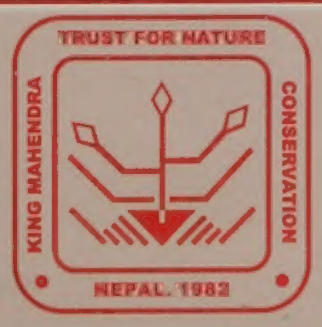

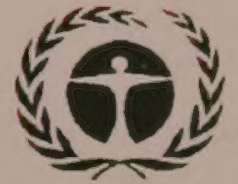

UNEP

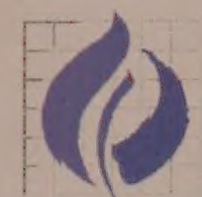

WCMC 
Digitized by the Internet Archive in 2010 with funding from UNEP-WCMC, Cambridge 


\section{Guidelines for Biodiversity Assessment and Monitoring for Protected Areas}



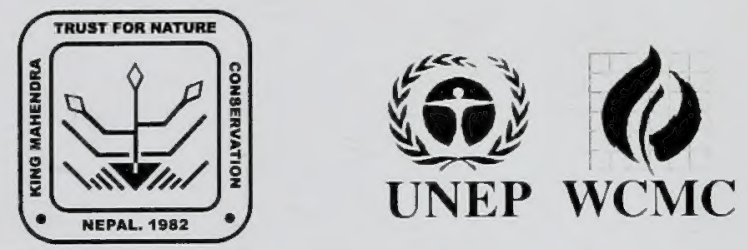

Copynght:

Citation:

Layout \& Printed by: Graphic Plus, Nepal \# 4267682

Available from:

Contact: Kathmandu, Nepal.

Information Unit

e-mail: info@kmtnc.org.np

\section{(C) 2005 KMTNC and UNEP-WCMC}

Reproduction of this publication for educational and other noncommercial purpose is authorised without prior written permission from the copyright holder provided the source is fully acknowledged.

Tucker, G., Bubb P., de Heer M., Miles L., Lawrence A., Bajracharya S. B., Nepal R. C., Sherchan R., Chapagain N.R. 2005. Guidelines for Biodiversity Assessment and Monitoring for Protected Areas. KMTNC,

King Mahendra Trust for Nature Conservation

P O Box 3712 Kathmandu, Nepal

The Guidelines are based on the practical experience of KMTNC and UNEP-WCMC. We welcome comments and suggestions. Please contact eitherinfo@kmtnc.org.np or info@unep-wcmc.org.

Cover Photo: Siddhartha Bajra Bajracharya

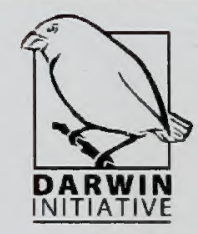

This publication has been produced with the support of the UK Darwin Initiative as part of project 163/11/020 "Building capacity for biodiversity monitoring and assessment in

Nepal". The Darwin Initiative is a small grants programme that aims to promote biodiversity conservation and sustainable use of resources in less developed countries. The Initiative is funded and administered by the UK Department for Environment, Food and Rural Affairs, (Defra).

http://www.darwin.gov.uk/index.htm 


\section{Table of Contents}

Acknowledgements

Foreword Office-in-Charge, UNEP World Conservation

Monitoring Centre

Foreword Vice-chairman, National Planning Commission

Foreword Member Secretary, King Mahendra Trust for

Nature Conservation

\section{Introduction}

$1.1 \quad$ Background to the guidelines 1

$1.2 \quad$ Use of the guidelines

2 Definitions and purpose of biodiversity assessment and monitoring for protected area management

2.1 Biodiversity assessment and monitoring as part of protected area management planning

2.2 What is a biodiversity assessment?

What is monitoring?

3 Carrying out a biodiversity assessment for a protected area

3.1 Defining the scope and approach of the assessment

3.2 Creating an enabling environment for participation

4. Developing a protected area biodiversity monitoring programme 
4.4 Define the location and boundaries of the biodiversity features to be monitored

4.5 Assess available monitoring resources and prepare budget 46

4.6 Determine the monitoring frequency 48

$4.7 \quad$ Select measurement methods $\quad 49$

4.8 Establish the appropriate time to carry out surveys 58

4.9 Devise a sampling scheme 58

4.10 Devise data recording forms and document methods 65

$\begin{array}{lll}4.11 & \text { Test methods } & 67\end{array}$

4.12 Review the monitoring programme in relation to available $\begin{array}{ll}\text { long-term resources } & 68\end{array}$

$\begin{array}{lll}4.13 & \text { Prepare a work plan } & 69\end{array}$

$\begin{array}{lll}4.14 & \text { Carry out necessary training } & 73\end{array}$

4.15 Analyse data $\quad 73$

4.16 Report results to stakeholders $\quad 80$

$\begin{array}{lll}4.17 & \text { Review the monitoring programme } & 80\end{array}$

5. References $\quad 82$

$\begin{array}{ll}\text { 6. Acronyms } & 87\end{array}$

$\begin{array}{ll}\text { 7. Glossary } & 88\end{array}$

8. Example protocols from the ACAP biodiversity programme 90

8.1 Introduction 90

8.2 ACAP Monitoring protocol for Snow Leopard 90

8.3 ACAP Monitoring protocol for Himalayan Griffon and $\begin{array}{ll}\text { other vultures } & 97\end{array}$

8.4 ACAP Monitoring protocol for broad-leaved forest habitat $\begin{array}{ll}\text { quality } & 107\end{array}$

8.5 ACAP Monitoring protocol for broad-leaved forest birds $\quad 114$

8.6 ACAP Monitoring protocol for remote sensing of habitat extent and quality 119 


\section{ACKNOWLEDGEMENTS}

We wish to thank the numerous people who contributed to these guidelines. We are especially grateful to the staff of the KMTNC-Annapurna Conservation Area Project who took part in or assisted with the various workshops and field training exercises, including:

Ajay Pandey - Conservation Officer, KMTNC-ACAP

Amar Gurung - Senior NRCA, KMTNC-ACAP, Lomanthang

Anil Bhattachan - CDA, KMTNC-ACAP, Jomsom

Arbin K.C. - NRCA, KMTNC-ACAP, Bhujung

Basu Dev Neupane - NRCA, KMTNC-ACAP, Lomanthang

Bhim Prasad Upadhya - NRCA, KMTNC-ACAP, Lwang

Bir Bahadur Thapa - NRCA, KMTNC-ACAP. Jomsom

Bishnu Prasad Paudel - NRCA, KMTNC-ACAP, Jomsom

Dhruba Laudari - NRCA, KMTNC-ACAP, Ghandruk

Dom Bahadur Pun, JTA, KMTNC-ACAP, Ghandruk

Gehendra B. Gurung - Director, KMTNC-ACAP, Pokhara

Hira B. KC - NRCA, KMTNC-ACAP, Lomanthang

Kaji Ram Adhikari - NRCA, KMTNC-ACAP, Bhujung

Kamal Thapa - NRCA, KMTNC-ACAP, Ghandruk

Krishna Gurung - TDA, KMTNC-ACAP, Jomsom

Kriti Nath Paudel - Conservation Officer, KMTNC-ACAP, Lwang

Lizan Kumar Maskey - Conservation Officer, KMTNC-ACAP, Jomsom

Mani Prasad Gurung - CEA, KMTNC-ACAP, Bhujung

Ms Hari Maya Gurung - GDA, KMTNC-ACAP, Lomanthang

Ms. Anu Kumari Lama - TD Officer, KMTNC-ACAP, Pokhara

Ms. Ganga Nakarmi - Gender Development Officer, KMTNC-ACAP, Pokhara

Ms. Min Maya Gurung - GDA, KMTNC-ACAP, Manang

Ms. Shanti Gurung - GDA, KMTNC-ACAP, Lwang

Naresh Subedi - Conservation Officer, KMTNC-BCP

Professor Karan B. Shah -Tribhuvan University, Natural History Museum

Raj Kumar Gurung - NRCA, KMTNC-ACAP, Manang

Rajendra Suwal- Senior Ornithologist, Kathmandu

Ram Prakash Singh - NRCA, KMTNC-ACAP, Sikles

Rishiram Sudedi - Ranger, KMTNC-BCC, Sauraha

Sailendra Kumar Yadav - TDA, KMTNC-ACAP, Lomanthang

Shankar Chaudhary - Sr. Ranger, KMTNC-BCC, Sauraha

Sherjung Gurung - CEA, KMTNC-ACAP, Manang

Shree Krishna Neupane- NRCA, KMTNC-ACAP, Manang

Shreeram Ghimire - Senior Programme Assistant, KMTNC-BCP, Bardia

Sunil Marsani - CEA, KMTNC-ACAP, Sikles

Suresh Thapa - Senior NRCA, KMTNC-ACAP, Jomsom

Surya Bahadur Pandey - Conservation Officer, DNPWC

Top Bahadur Khatri, Project Manager, HMG/UNDP/PCP

The project was conceptualised and designed by Adrian Newton (University of Bournemouth, formerly UNEP-WCMC) and Siddhartha Bajra Bajracharya (KMTNC). We are also grateful for advice and information provided by other staff of UNEP-WCMC and KMTNC, including Tim Inskipp, and by Carol Inskipp, Mark Eton (RSPB) and Peter Garson (Chair, WPA/BirdLife/SSC Pheasant Specialist Group, c/o University of Newcastle). We also thank RPS and Scottish Natural Heritage for permission to use figures from their Handbook of Biodiversity Methods: survey, evaluation and monitoring (Hill et al. 2005). 


\section{Foreword by the Officer-in-Charge, UNEP- World Conservation Monitoring Centre}

Biodiversity plays a central role in our lives. We depend upon plants and animal species for food, medicines and raw materials. The genetic resources contained within biodiversity hold the basis of our continued existence. The services provided by biodiversity and ecosystems helps to sustain our livelihoods and protect our health. And there is no doubt that the beauty and variety of our living species greatly improve the quality of our lives.

There has been increasing global recognition of the importance of biodiversity. The 2002 World Summit on Sustainable Development reaffirmed the critical importance of biodiversity in maintaining our wellbeing but also acknowledged that it was being lost at an alarming rate. In response, it established a target of significant reduction by 2010 in the rate of biodiversity loss, regarding this as one of the most important milestones in progress towards a sustainable future. However, overall this recognition has yet to be transformed into concrete action on the scale that will help us achieve the 2010 .

The UNEP World Conservation Monitoring Centre (UNEP-WCMC) provides information on the status of the world's living resources, from plants and species to the ecosystems that house them, in order to promote better informed decision-making and support sustainable management of biodiversity. These Guidelines are a significant contribution to our goals, as they help the managers of protected areas obtain and use the biodiversity information necessary for their work. This information will help them know if their actions are being effective, to prioritise their resources, and to promote their successes.

The Guidelines are based on the practical experience of KMTNC and UNEP-WCMC in the development of a biodiversity monitoring system for the management of the Annapurna Conservation Area. They are the result of a productive collaboration and we hope that they will be of wide relevance for protected area managers throughout Nepal and beyond. I would like to acknowledge the commitment of the King Mahendra Trust for Nature Conservation to the success of this project and the support of the Darwin Initiative of the UK Government, which have made these results possible. It is only through such collaboration that we can use the expertise gained in actual practice to help implement the policies expressed at national and international level and to bring about real change that is vital to our future and that of our children.

Mr. Kaveh Zahedi

Officer-in-Charge

UNEP-World Conservation Monitoring Centre

Cambridge, UK 


\section{Foreword by the Vice-Chairman, National Planning Commission of Nepal}

As we move on to the $10^{\text {th }}$ Five Year Plan, a significant area of Nepal $(18 \%)$ is in some form of protected area - national parks, wildlife reserves, hunting reserve and conservation areas. There is an extensive shift in protected area management approach over the period from strict protection approach to community-based conservation approach. With the changes in the management approach, protected areas management has been gradually and successfully linked to local livelihood. This is a remarkable achievement for Nepal and a demonstration of importance accorded to biodiversity conservation in Nepal.

Until recently, however, it has been difficult to monitor biodiversity in protected areas in an objective and scientifically rigorous way. Therefore, there is a growing realization on need for blending biodiversity assessment and monitoring system within a protected areas management system to achieve sound and effective management of protected areas. The present Guidelines to Biodiversity Assessment and Monitoring prepared through the practical experience in Annapurna Conservation Area should help in effective monitoring of biodiversity in protected areas. The Guidelines will be an important document for the managers of protected areas in Nepal and else where.

I would like to congratulate the King Mahendra Trust for Nature Conservation (KMTNC) and its partner organisation the UNEP-World Conservation Monitoring Centre for producing the Guidelines document and extend my appreciation to those who contributed to this document. I would also like to acknowledge the support of the Darwin Initiative of the UK Government.

Shankar P. Sharma, PhD

Vice-Chairman

National Planning Commission

Singh Durbar, Kathmandu 


\title{
Foreword by the Member Secretary, King Mahendra Trust for Nature Conservation, Nepal
}

The King Mahendra Trust for Nature Conservation (KMTNC) in collaboration with the United Nation's Environment Programme (UNEP)-World Conservation Monitoring Centre (WCMC) has developed 'Guidelines for Biodiversity Assessment and Monitoring for Protected Areas'. With the mission to conserve, manage and promote nature in all its diversity balancing human needs, KMTNC has been actively working in biodiversity conservation in Nepal for more than two decades. The document published in partnership with UNEP-WCMC is an initiative to contribute in effective management of protected areas in Nepal.

With the establishment of protected areas since the last three decades, Nepal has made remarkable achievements in conserving its rich biological diversity and cultural heritage. KMTNC, over the years, with support from His Majesty's Government, has developed a new and innovative concept for protected area management effectively linking conservation with local livelihood. I hope the guidelines will be a tangible tool for biodiversity assessment and monitoring in protected areas.

I praise the input of the team of KMTNC and UNEP-WCMC and wish their endeavour a success. While it is difficult to name few out of a core team, I acknowledge the effort of Dr. Siddhartha Bajra Bajracharya, Mr. Gehendra Gurung, Mr. Ram Chandra Nepal and Mr. Nawaraj Chapagain for successfully designing and implementing the Darwin Initiative funded project.

I also take this opportunity to extend my appreciation to those who contributed to this document and acknowledge the support of the Darwin Initiative of the UK Government.

\author{
Mr. Arup Rajouria \\ Member Secretary \\ King Mahendra Trust for Nature Conservation \\ Jawalakhel, Lalitpur \\ Nepal
}




\section{Introduction}

\subsection{Background to the guidelines}

These guidelines are a product of the project "Building capacity for biodiversity assessment and monitoring in Nepal". This was a joint project between the UNEP World Conservation Monitoring Centre (UNEP-WCMC) and the King Mahendra Trust for Nature Conservation (KMTNC), and was funded by the Darwin Initiative of the UK government from 2002 to 2005. The principal goal of the Darwin project was to strengthen the capacity of KMTNC to include biodiversity information in management decisions of the Annapurna Conservation Area Project (ACAP). These guidelines have been developed through the Darwin project's training courses and the field testing of the monitoring protocols by KMTNC staff.

The King Mahendra Trust for the Nature Conservation (KMTNC) was established in 1982 by a Legislative Act of the Parliament of Nepal, which mandated it as an autonomous, nonprofit and non-governmental organisation, to work in the field of nature conservation in Nepal. KMTNC's mission to promote, manage and conserve nature in all its diversity in Nepal is supported by the following guiding principles: (a) always maintaining a balance between human needs and the environment to guarantee long-term sustainability; (b) always seeking maximum community participation in which locals are recognised both as principle actors and beneficiaries; (c) always linking economic, environmental and ethical factors in conservation activities; (d) always managing operations based on sound economic principles and (e) always aiming for quality in all activities.

Geographically, KMTNC activities are spread from the tropical plains to the high Himalayan regions, including Trans-Himalayan regions. The Annapurna Conservation Area (ACA) is one of the major initiatives of KMTNC in the High Himalayan and the Trans-Himalayan regions.

\section{Box 1.1. Annapurna Conservation Area and its importance}

Annapurna Conservation Area Project, launched in 1986, is the largest undertaking of KMTNC, and the first and largest Conservation Area in Nepal. ACA is located in the Mountain regions of the west-central Nepal at latitude $28^{\circ} 50^{\prime} \mathrm{N}$ and longitude $83^{\circ} 57^{\prime} \mathrm{E}$ (Figure 1.1). ACA covers an area of $7,629 \mathrm{sq} . \mathrm{km}$. and is home to over 120,000 local people of different ethnic. cultural and linguistic groups. ACA is rich in biodiversity and is a treasure house for 1,226 species of plants, 38 species of orchids, 9 species of rhododendrons, 101 species of mammals, 474 species of birds, 39 species of reptiles and 22 species of amphibians. It harbours rare and endangered wildlife species such as the Snow Leopard. Musk Deer, Tibetan Argali. Impeyan Pheasant and Tragopan Pheasant.

ACA is well known internationally and in Nepal for its beautiful mountains and a unique ecology. The area is bounded to the north by the dry alpine deserts of Dolpo and Tibet, to the

(cont.) 


\section{Box 1.1. Annapurna Conservation Area and its importance (cont.)}

west by the Dhaulagiri Himal, to the east by the Marshyangdi Valley and to the south by valleys and foothills surrounding Pokhara. Some of the world's highest snow peaks over 8,000 $m$ and the world's deepest valley of the Kali Gandaki river are in ACA. These extreme diversities have made it Nepal's most popular trekking destination with over 70,000 trekking tourists in the year 2000 , which is over 62 per cent of the total trekking tourists visiting Nepal.

ACA is a new model of protected area in Nepal where local communities are involved in protected area management. KMTNC pioneered the ACA concept, realising that protected areas cannot be isolated from the people living in and around them. The sustainable use of local resources, particularly forest, remains integral both to the livelihoods of the local communities and to the conservation of biodiversity and fragile environments. The local community's role as a partner in the management of a conservation area through a Conservation Area Management Committee has been explicitly reflected in the Consevation Area Management Regulations (CAMR). The regulations authorise Conservation Area Management Committees to issue permits and collect revenues from the local community for allowing fishing, forest resource utilisation, grazing and other resources utilisation. The Conservation Area Management Committee (CAMC) is the main executive body constituted by the KMTNCACAP to manage the conservation area. The villagers of every ward nominate nine of the 15 members. Committees exist in all the 55 Village Development Committees of ACA and under these committees are several grassroots institutions, such as the forest management committees, mother's group, tourism management committees, electricity management committee, etc. All these institutions are responsible for executing and linking their specific activities with the conservation of natural resources.

The management of ACA is based on the participatory multi-land use protected area concept. To balance global biodiversity conservation goals and local livelihood concerns, an integrated conservation and development approach has been adopted. A Management Plan prepared in 1997 was based on eight management goals, with objectives, priority programmes and policies. These management goals were: i) to build and strengthen the institutional capacity of ACAP through human resource development; ii) to develop a long term framework for conservation of the natural resources in ACA; iii) to promote nature conservation through sustainable development of tourism; iv) to enhance the status of women by according an equal role to them in decision making processes in conservation and sustainable development; v) reduce stress on critical resources primarily forests through wider use of micro hydro electricity and other alternative programmes; vi) to promote community infrastructure development; vii) to promote cultural heritage conservation; and viii) to carry out essential multi-disciplinary management research to support conservation and development initiatives. 


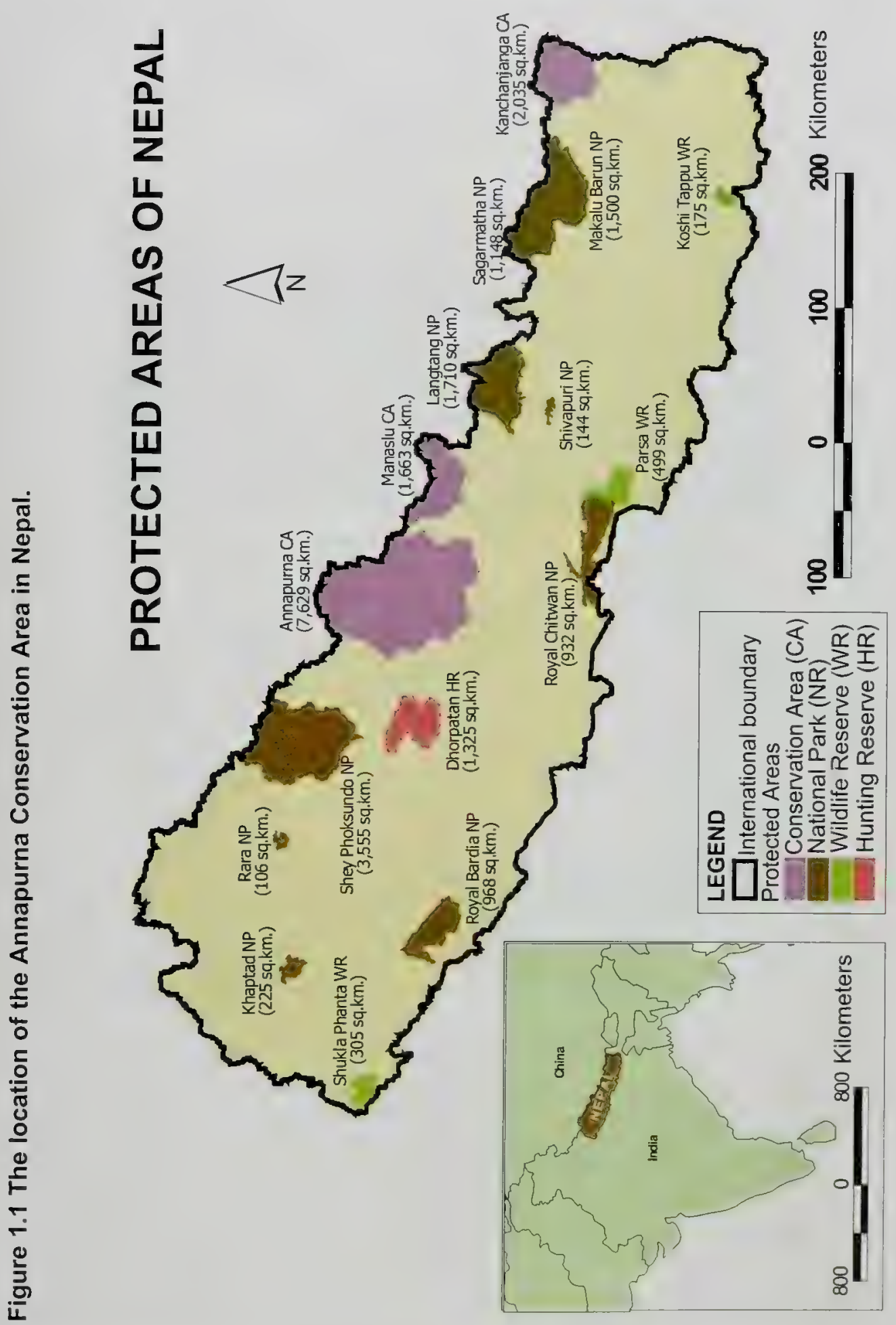


Figure 1.2 Major habitat types in the Annapurna Conservation Area

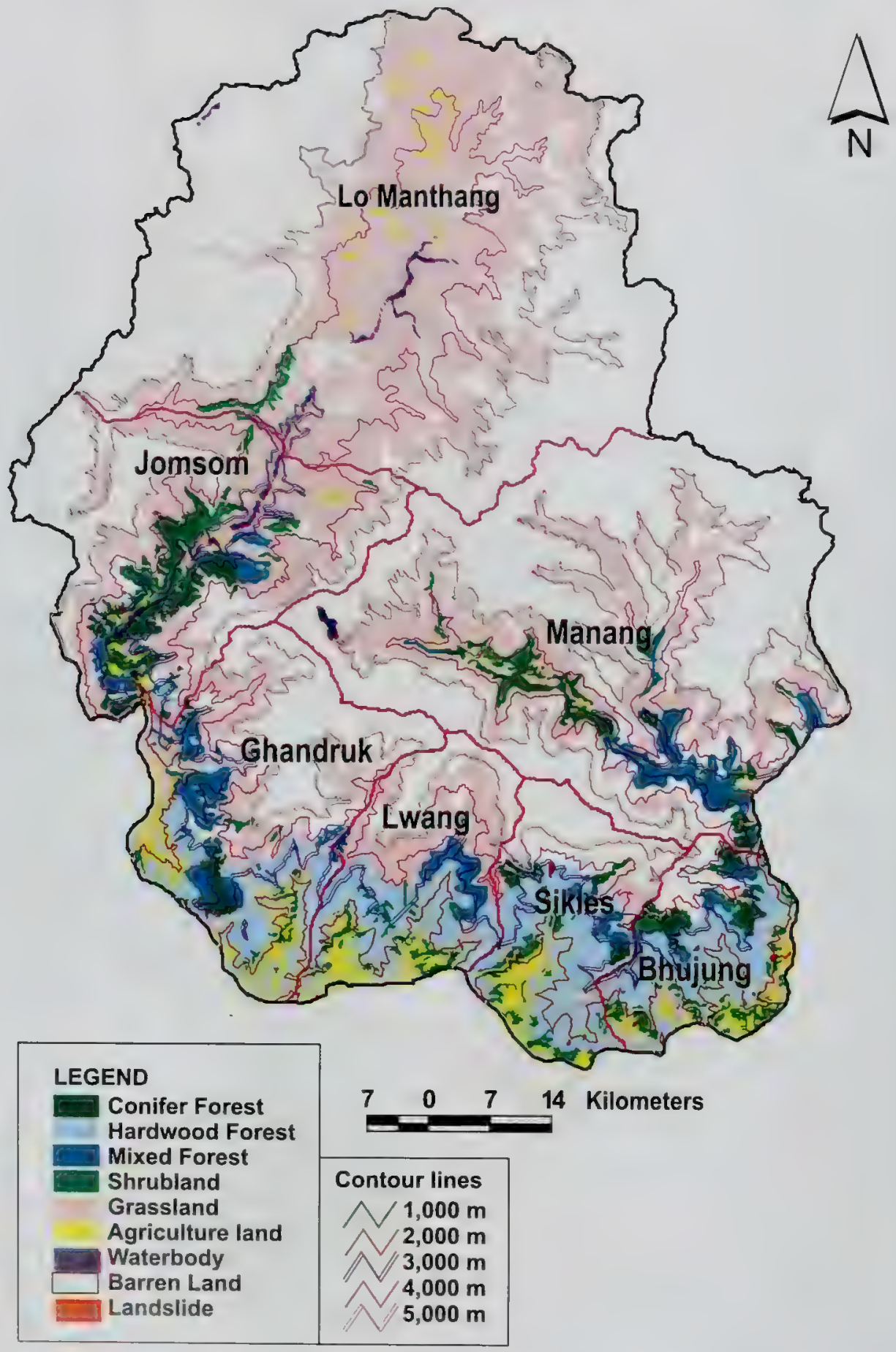


These guidelines have been developed with the management staff of the Annapurna Conservation Area Project and are designed for their use. Whilst the examples refer to the management of a mountain protected area, it is anticipated that much of the generic advice on establishing assessment and monitoring programmes will be applicable to all types of protected areas.

\section{Plate 1.1 A typical landscape of Annapurna Conservation Area}

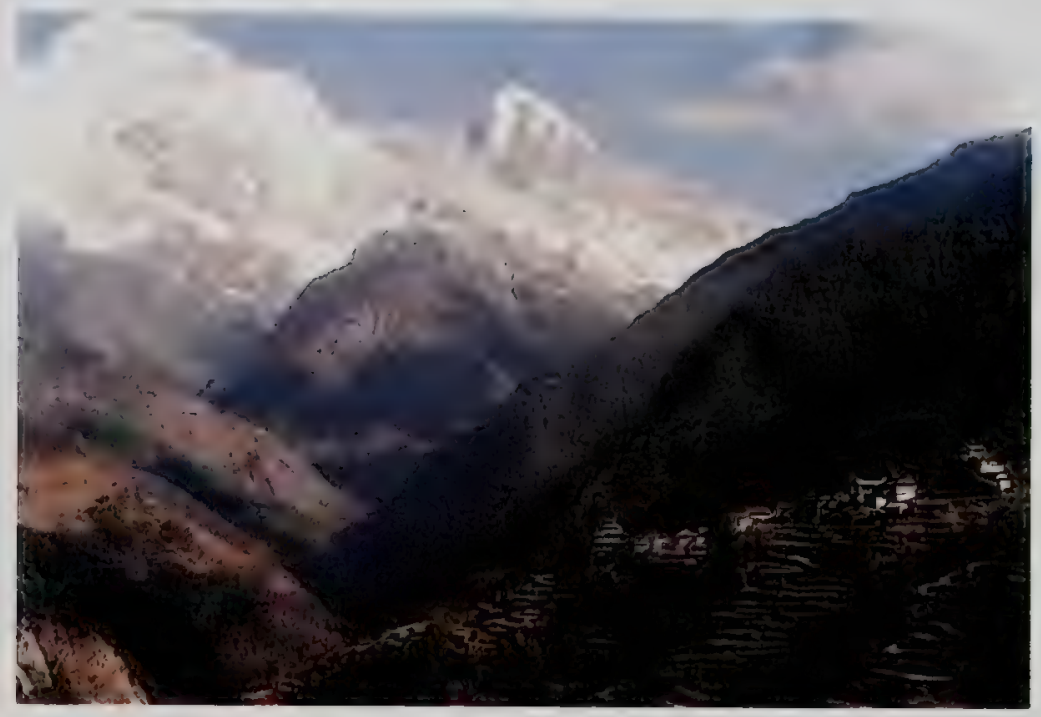

\subsection{Use of the guidelines}

Biodiversity assessment and monitoring in protected areas is normally, and most appropriately, carried out as part of a management planning process. It is therefore suggested that these guidelines are read and used in conjunction with appropriate guidance on management planning within protected areas. However, in some protected areas, management plans may not have been prepared, or may not deal with biodiversity issues in detail (such as in the ACA up to now). Some advice is therefore given in Chapter 2 on key aspects of management planning so that biodiversity assessments can be carried out and monitoring programmes established in the absence of a detailed biodiversity management plan.

An introduction to biodiversity assessments, with emphasis on participatory approaches is given in Chapter 3, however, it is beyond the scope of these guidelines to give detailed practical advice on this subject, and therefore the reader is directed to some recommended references for further information.

Chapter 4 provides guidance on the key practical considerations and decisions involved in establishing a protected area monitoring programme. This primarily focuses on issues concerned with selecting field methods and survey sampling strategies that would be carried out by trained staff or contractors. However, many of the key principles, such as those associated with sampling, may also be applicable to participatory approaches (e.g. selection of sample villages for holding interviews). The chapter includes some brief advice on statistical analysis of monitoring data, but again this subject is too large to deal with in detail here. Tables are therefore provided that give guidance on appropriate statistical tests for various situations, and sources of further information, and statistical software. These should enable readers to complete most forms of statistical analysis required for monitoring purposes. 


\section{Definitions and purpose of biodiversity assessment and monitoring for protected area management}

\subsection{Biodiversity assessment and monitoring as part of protected area management planning}

One of the principle reasons for creating National Parks and other types of protected area is to conserve the special biodiversity values within them. Effective conservation of this biodiversity normally requires management actions that are best carried out within some form of management planning framework. This is most efficient if carried out as a continuous process, where plans are prepared, implemented, reviewed and revised according to their impacts as established by monitoring (see Figure 2.1).

Figure 2.1. A simplified management planning cycle

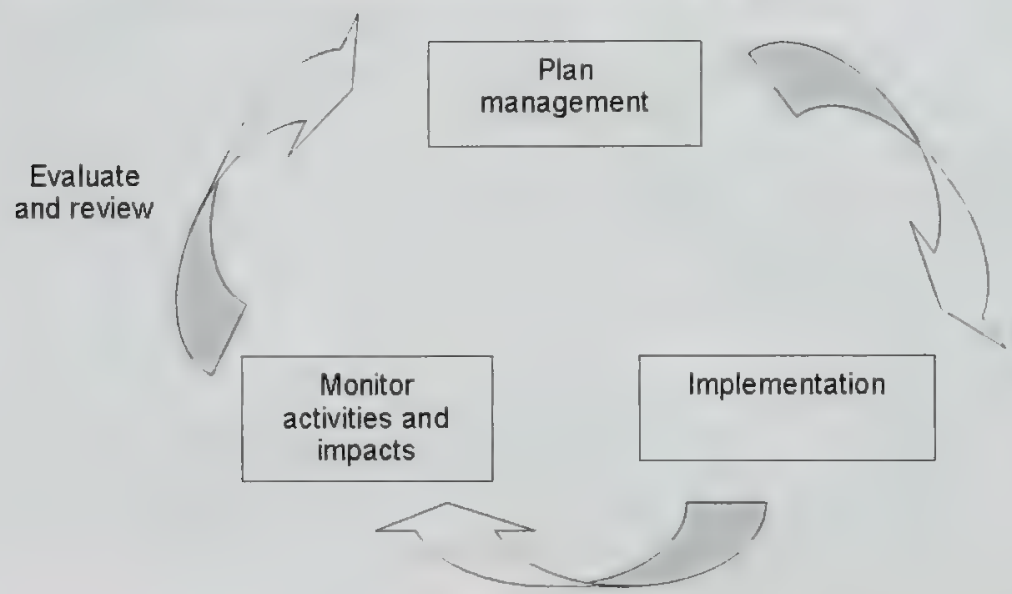

Source: Adapted from Thomas \& Middleton 2003

To develop an effective management plan requires an initial assessment of the status of biodiversity, to set the priorities and objectives for management, and then ongoing monitoring, to establish whether or not management actions are achieving their objectives. Biodiversity assessments, therefore, normally form key components of protected area management plans, from which monitoring strategies and programmes are identified and implemented, as depicted in Figure 2.2. The process is essentially a circular one, with periodic evaluations being carried out to assess progress in the implementation of actions and the achievement of objectives. Over the long-term the monitoring data should also be used to re-evaluate the most recent biodiversity assessment to ensure that decisions are based on the best and most up-to-date information. 
Figure 2.2. Biodiversity assessment and monitoring within a management planning cycle

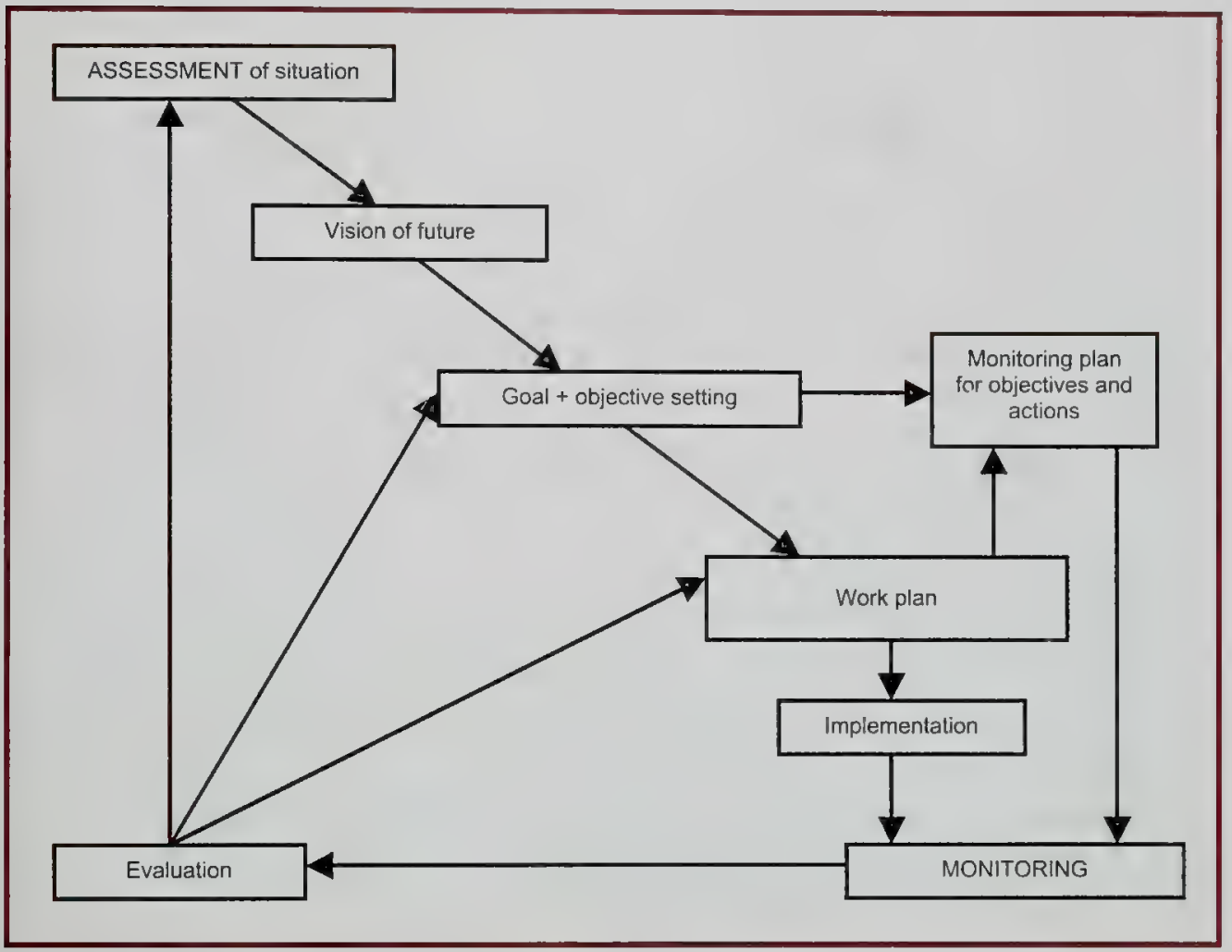

Four key principles for effective management planning have been identified by Hockings et al. (2001):

1. The plan should have conceptual rigour as a decision making framework. This framework should provide:

- a clear sense of a desired future for the area;

- a set of strategies and actions for achieving this future;

- clear guidance that can assist managers dealing with opportunities and eventualities that arise during the life of the plan;

- a basis for monitoring of plan implementation and progress towards the desired future and adjustment of planning strategies and actions as required.

2. The plan should place the management of the area into a relevant environmental, social and economic planning context. Where possible, planning decisions should be integrated into this broader planning framework.

3. The content of the plan should be formulated within an adequate and relevant information base and should place management issues within a broader context and in relation to the desired future for the area: the needs and interests of any local and indigenous communities and other stakeholders should have been considered within the plan.

4. The plan should provide a programme and prioritised set of actions for achieving the desired future for the area. 
Protected areas cannot remain in isolation from the communities and the economic activities in and around protected area. As noted in point 3, plans should address the needs of local communities and other stakeholders. The $\mathrm{V}^{\text {th }}$ IUCN World Park Congress held in Durban, South Africa has also emphasised on the rights of local communities in relation to natural resources and biodiversity conservation. Participatory management approaches, 'such as where "no or" more social actors negotiate, define and guarantee amongst themselves a fair sharing of the management functions, entitlements and responsibilities for a given territory; area or set of natural resources" (Borrini-Feyerabend et al. 2000) should therefore be used wherever appropriate. At the very least management planning should involve adequate consultations with all stakeholders.

Management plans for protected areas are typically prepared following a logically ordered sequence as summarised below (based on the IUCN Guidelines prepared by Thomas and Middleton 2003).

1. Pre-planning phase (appointment of planning team, scoping of task, agreement on the process to be followed).

2. Data gathering and review.

3. Evaluation of data and resource information.

4. Identification of constraints, opportunities and threats.

5. Development of overall long-term vision for the protected area and specific objectives.

6. Development of options for achieving the vision and objectives (including zoning if appropriate).

7. Preparation of a draft management plan.

8. Public consultation on the draft management plan.

9. Assessment of submissions, revisions of draft management plan, production of final management plan and reports on consultation process.

10. Approval / endorsement of management plan.

11. Implementation of actions identified within the management plan.

12. Monitoring and evaluation of implementation and impacts of the management plan.

13. Review and update of the management plan.

In practice some of these steps may be carried out iteratively (i.e. by going back and forth). For example, collation of data on the protected area (Step 2) may be influenced by an evaluation of what features are of particular value (Step 3). The proposed sequence also suggests that consultations are carried out at Stage 8 . However, we recommend that consultations start at Stage 1 and should involve community participation at Stages 2, 3, 4, 5 and 6 to ensure that the plan is adequately researched and that it deals with the interests of all stakeholders.

It is important to note that the proposed sequence is circular, such that Stage 13 is a review and up-date of the management plan. This facilitates adaptive management. The key rationale for adaptive management of biodiversity is the recognition that our knowledge of ecological

Also known as co-management, collaborative, joint. mixed, multi-party or round-table management. 
relationships is incomplete and, therefore, the management of natural resources is always experimental. It therefore aims to improve our management effectiveness by studying the impacts of implemented activities and learning from these. Adaptive management therefore explicitly states objectives (and hypotheses on how they are to be achieved), monitoring requirements and evaluation methods, and then adjusts and improves actions according to the results obtained and lessons learnt. See BC Forest Service at http://www.for.gov.bc.ca/ hfp/amhome/introgd/toc.htm and the website of Foundations of Success http:// www. fosonline.org/Resources. ffm for further guidance on adaptive management.

There are a large number of suggested contents, structures and formats for protected area management plans (e.g. Ramsar Bureau 2002), but there is actually considerable similarity amongst them. According to Thomas and Middleton (2003) the most commonly found contents of a management plan include:

- Executive summary.

- Introduction (e.g. purpose and scope of plan, reason for designation of protected area and authority for plan).

- Description of the protected area.

- Evaluation of the protected area.

- Analysis of issues and problems.

- Vision and objectives.

- Zoning plan (if appropriate).

- Management actions (list of agreed actions, identifying schedule of work, responsibilities, priorities, costs and other required resources).

- Monitoring and review.

In Nepal a national framework for management plans has been agreed, which is summarised in Box 2.2.

\section{Box 2.2. A national framework for management plans}

1. Executive summary

2. Introduction and background

3. Rationale of a management plan

4. Park management

5. Buffer zone management

6. Budget and action programmes
Introduction, statement of significance, description of protected area, legislation and policies, present practices

Goals and objectives, vision and mission statement, guiding policies, management approaches and logical framework of outputs

Management zones, management issues, strategies and actions

Management zones, management issues, strategies and actions

Budget summary, park and buffer management activities and budget schedule for 5 years 


\subsection{What is a biodiversity assessment?}

Biodiversity assessment is the first stage in the process of defining the biodiversity management objectives for an area. Its purpose is to gather and assess the information required to make decisions and recommendations for the future.

In the context of management planning for a protected area a biodiversity assessment involves measuring or surveying what exists in the area and what is known about it, judging its value and identifying the most important features (e.g. grasslands for livestock grazing, timber for fuel and building materials, medicinal plants, water storage functions and habitats and species of particular conservation concern). Assessments therefore need to involve a social component that identifies biodiversity features of high socio-economic value, as well as features of high aesthetic, cultural or intrinsic value. Assessments also typically include identification of the principal factors affecting the important biodiversity features within the protected area (e.g. the dependency of top level predators, such as a Snow Leopard Uncia uncia, on its prey species, or the impacts of fuel wood collection on forest regeneration).

\subsection{What is monitoring?}

Monitoring is often thought of as a programme of repeated surveys or measurements, usually by means of a standardised procedure. However, this is merely surveillance if there is no predetermined objective or value that guides what the findings ought to be. For example, daily measurements of rainfall are a type of surveillance. It is more appropriate for protected area management needs to define monitoring more rigorously as: "the collection and analysis of repeated observations or measurements to evaluate changes in condition and progress toward meeting a management objective" (Elinga et al. 2001).

Thus, in the context of protected area management needs, monitoring is carried out to determine if biodiversity conservation, livelihood enhancement and other objectives are being met, such as the maintenance of the existing area of a particular habitat or a specified number of a particular species, or development of a community woodlot to reduce impact on natural forests. It is better to think of monitoring in this more precise way, because it helps to ensure that protected area monitoring programmes and their methods are focused on protected area objectives, and so support their achievement. Thus, a protected area monitoring programme has a specific purpose, tied to objectives that have already been defined.

Monitoring should not attempt to describe the general ecology of a site or measure things that may merely be of interest. Unfortunately, monitoring schemes often resort to measuring a wide variety of variables, which may or may not be related to the protected area objectives and management questions that need to be addressed. As a result, time and money may be spent collecting unnecessary data. Even worse, it may be found that key management questions cannot be answered with the information obtained.

Nor should monitoring programmes be confused with research studies that are designed to establish why something is happening (i.e. to test a hypothesis). Many of the field methods and scientific principles of biodiversity assessment and monitoring can be used in research, but their purpose is different. In particular, research may often need to be more detailed, sensitive and scientifically rigorous than required for many monitoring purposes. For example, it may be adequate to monitor vulture numbers by occasional counts of soaring birds. This may establish if population trends are meeting conservation objectives, but will 
not reveal the factors determining population size. To establish what influences population size would require much more time consuming, difficult and costly studies. These would probably not be necessary if, according to monitoring data. populations appear "healthy" and conservation objectives are being met. However, such detailed research could be triggered if monitoring data reveal a decline below a preset warning level (which should be above the conservation objective population level).

In practice, monitoring data may sometimes be of use for research work. For example, if it is necessary to measure livestock and vulture numbers within the same area, then it may be possible to examine if vulture numbers are affected by livestock numbers (by correlation analysis). However, such fortuitous use of monitoring data should not influence monitoring designs. Instead monitoring and research requirements should be designed separately and then if there is overlap between requirements (e.g. both need the same data on livestock numbers) then they may be combined.

\section{Plate 3.1 A female Cheer Pheasant}

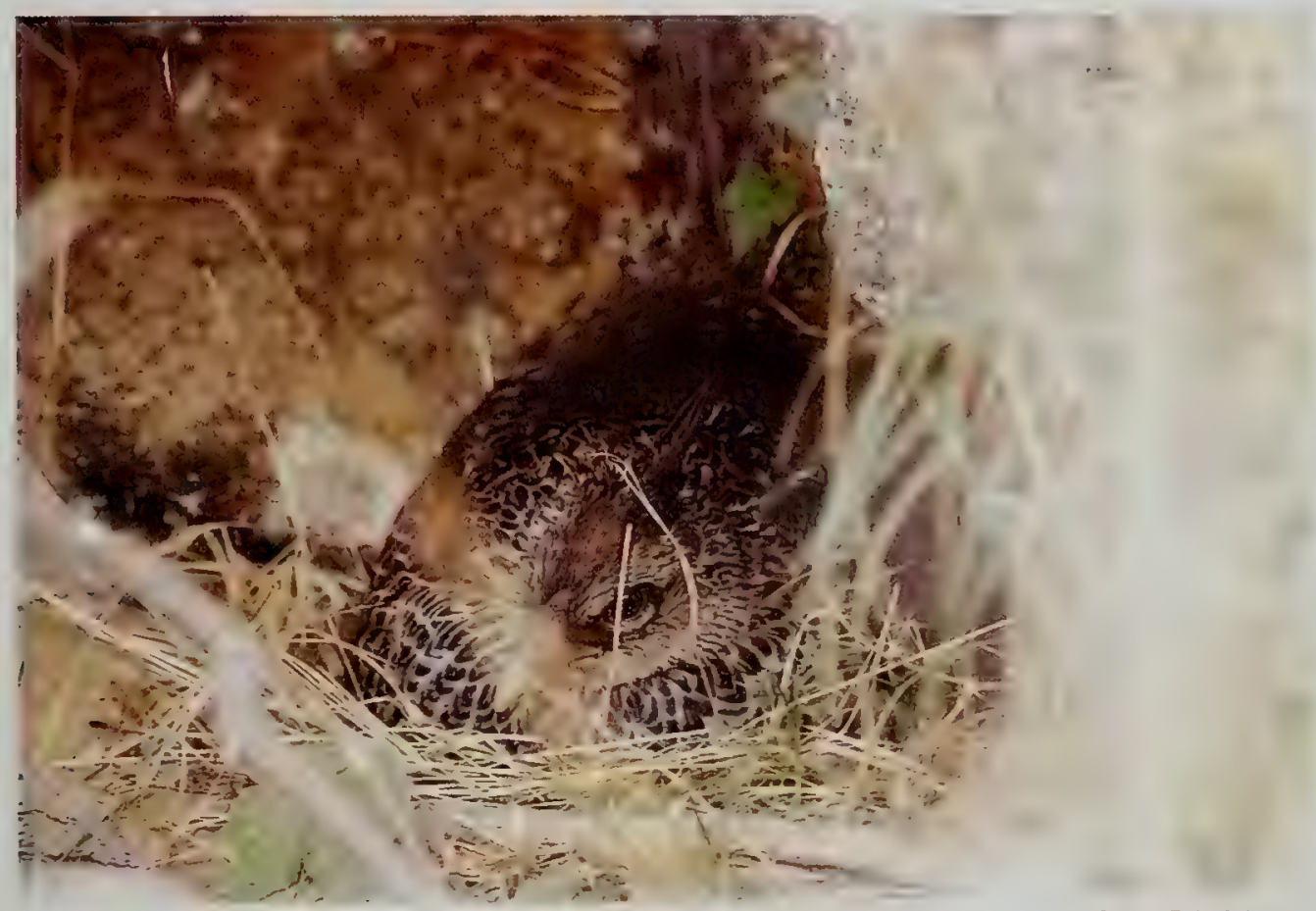

Photo: Raju Acharya 


\section{Carrying out a biodiversity assessment for a protected area}

A biodiversity assessment typically involves a number of key steps as outlined in Figure 3.1 (which are analogous to Stages 2-4 of the management planning process outlined in Section 2.1). The assessment may then lead on to the setting of broad goals, aims and objectives for the protected area. These key steps are further described in the following sections.

\section{Figure 3.1. Defining the scope $\&$ approach of the assessment}

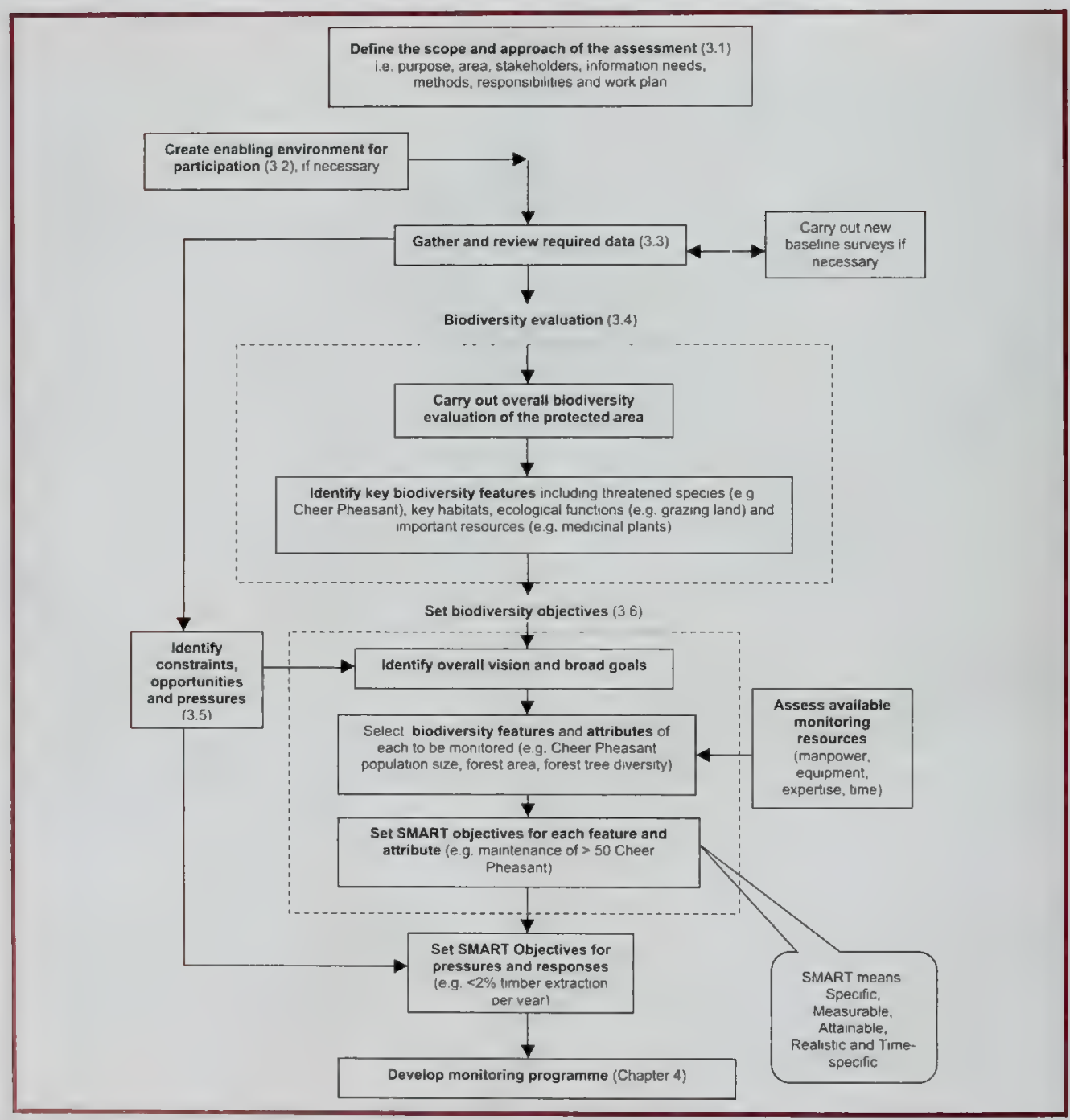

Note: figures in brackets refer to relevant text sections 


\subsection{Defining the scope and approach of the assessment}

\subsubsection{Defining the scope}

A biodiversity assessment could potentially cover an enormous range of questions and require a huge investment in data gathering and analysis. It is therefore necessary to define the scope of the assessment. It should be focused on producing the information needed for specific decision-making and planning purposes.

The stages in setting the scope and objectives of the assessment are:

1. Determine the purpose of the assessment, e.g. it may form part of the development of a management plan (as described in Section 2.1) or it may have a separate specific purpose. If it is for a management plan, then ensure that the szope and type of management is well defined and understood, e.g. a conservation plan for the ACA, or a Village Conservation Area Management Operational Plan.

2. Define the limits or boundaries of the area to be assessed. For example, the whole of the ACA or sub-units of this, such as the village land under the responsibility of a Conservation Area Management Committee (CAMC).

3. Determine who the stakeholders are in the assessment and what their information needs are (see Box 3.1). In particular, identify the biodiversity and management issues and priorities of the decision makers and other stakeholders. This will help establish which questions the assessment will need to answer (e.g. which species are of highest conservation importance, and which species are most important to local people and their livelihoods?).

4. Determine who may hold the information required to complete the assessment.

5. Select and agree the methods, responsibilities and work schedule for the assessment.

\section{Box 3.1. Identification of stakeholders and their information needs}

In the context of protected areas, stakeholders are likely to include:

- Landowners, home owners and occupiers (e.g. tenant farmers), including those in adjacent areas that may be affected by activities within the protected area.

- Leaders of local communities (e.g. CAMCs and VDCs in ACA).

- Businesses within the protected area (e.g. forestry, tourism, water supply), including owners and employees.

- Visitors to the area and those who organise such visits.

a Researchers with sites or projects within the protected area.

- Governmental, regional and local authority officials.

a Protected area authority staff.

A useful participatory process cannot begin until the stakeholders understand and respect each others' objectives and values. Usually an assessment facilitator will be needed to help begin this process.

The information need of each stakeholder is likely to depend on their perception of whether and why the area should be managed. For some, maintenance of livelihood will be most

(cont.) 


\section{Box 3.1. Identification of stakeholders and their information needs (cont.)}

important, for others, protection of culturally or spiritually important places, while others may be motivated by a concern to protect threatened species for all humanity. Thus, each stakeholder works with a set of assumptions, or values, about what is important, and it is these that influence both decisions about what is important in the protected area, its management objectives and evaluations of whether management has been successful or not. And it should also be remembered that different value-laden needs can also exist within stakeholder groups, including conservationists (Callicott et al. 1999) and local communities (Salim et al. 2001). Facilitators, therefore, need to recognise what is important to each stakeholder, to help them define their information needs.

\subsubsection{The benefits of a participatory approach}

As discussed in Chapter 1, protected area management planning should be undertaken using participatory approaches wherever possible, and this also applies to biodiversity assessments, whether part of a management plan or not. 'Participatory' in this context is often understood to mean involvement of rural communities, but can also involve other stakeholders such as students, policy makers, conservationists or volunteers. It can refer to scientists and local people working together to assess biodiversity, so that they understand each other's perspectives better. Participatory monitoring is a powerful approach that can improve the effectiveness of information gathering and help people understand the reasons for certain management decisions. It is therefore increasingly being used to support biodiversity conservation and management.

Advice on participatory assessment and monitoring in these guidelines draws on shared experience from an internet conference (Lawrence 2002) and published case studies. It is a new field, and much of the experience is from developing countries, where a participatory approach is particularly appropriate, but the processes would be similar in other contexts. Participation ranges from passive participation, where people are only told what is going to happen (and responses are often ignored), to self-mobilisation, where people take initiatives independent of external institutions (Pretty 1994). To date, most examples of participatory biodiversity assessment and monitoring reach only the halfway point in this range: people participate by providing labour so that data can be gathered more quickly and cheaply. Interactive participation where people contribute to decisions in biodiversity management, or self-mobilisation where they have full rights and responsibilities in biodiversity management, are still very rare. The current management structure within ACAP already enables interactive participation by local communities in management decision making, and therefore this existing framework can be expanded to include biodiversity assessment and monitoring as well.

Although the use of participatory approaches may complicate issues and be time consuming, their disadvantages are exceeded by their benefits. Local people are valuable participants in assessing and monitoring biodiversity, because:

1. They may have knowledge about wildlife, plants and resources derived from generations of use. For example, knowledge of the medicinal properties of some plants may be undocumented and known only to the local communities. 
2. Most monitoring systems within protected areas focus on protected species of wildlife and plants. Monitoring local resource use is a neglected but crucial dimension in planning sustainable harvesting by local people.

3. It is internationally acknowledged that involving local people in the planning and management of biodiversity and resources can increase their awareness and motivation for conservation. It can enhance an exchange of local and outside perceptions on the relationship between biodiversity and use patterns, leading to feedback on how to change unsustainable resource use practices.

4. The basis of decisions on biodiversity management, especially in protected areas, is often unclear to local communities depending on those resources. The involvement of local people in the gathering and analysis of biodiversity data will enable local communities to understand why certain decisions are made. This is likely to be particularly helpful when difficult choices need to be made that may appear to be detrimental to a local community's immediate interests (e.g. restrictions on resource usage to allow recovery).

5. Communication among stakeholders is often limited. Interacive participation by various partners, including nearby communities and protected area staff can improve relations (Fabricius and Burger 1997; Van Rijsoort and Zhang 2002), and resolve conflict (Bliss et al. 2001).

6. Particularly in developing countries, resources for biodiversity assessment are limited - human capacity, money and time are all scarce (Danielsen et al. 2000). Biodiversity monitoring and management systems should therefore be based on locally available capacity and resources to be sustainable.

However, it is important to recognise that there may be significant practical constraints on participation by local communities. Some communities within protected areas may be unaware of the relevance of appropriate protected area management for them, and hence its potential benefits. They may, therefore, have no incentive to participate in management planning. They may also have constraints on their time and inputs, especially if they are living on a subsistence or near-subsistence basis where all time is used on basic life-supporting activities.

It may therefore be necessary to precede participatory management planning, assessment or monitoring initiatives with basic socio-economic development, and awareness activities to inform communities of the potential benefits of participation. Training will also often be necessary. If stakeholders are to play a full role in management planning they will need to understand its aims, who the decision makers are and how the management planning system works.

\subsection{Creating an enabling environment for participation}

The time needed to facilitate a participatory process in biodiversity assessment and monitoring must not be underestimated. The process may take much longer than a nonparticipatory approach, but this investment is essential for building mutual understanding. to obtain useful data, and to promote local empowerment.

Before entering into a participatory process of biodiversity assessment and monitoring, an enabling environment is needed - i.e., favourable policy and institutional factors. In particular, decentralised decision making is required rather than top-down management (as has, for example, often been typical of the forestry sector in many countries). But where protected areas are strictly protected, the possibilities for interactive participation 
by surrounding communities may be limited, since the benefits perceived by these communities may not be high. In cases where the rules and regulations of the protected area enable sustainable use of resources and even involvement in management of the protected area, as in the ACA, incentives for local communities to participate in biodiversity and resource management planning, conservation and monitoring will be higher.

People who are leading or facilitating assessments need to be aware of any obstacles perceived by stakeholders before entering into the process, in order to address misunderstandings or justified fears. For example, in Yunnan, China, villagers were initially reluctant to join in, fearing that the monitoring process would lead to further restrictions in their resource use. This fear appeared to be justified during the analysis phase, when most of the proposed solutions involved banning resource use. More constructive solutions that provided benefits for all stakeholders had to be thought of, including sustainable resource use and enrichment planting (Van Rijsoort and Zhang 2002).

It is also important that facilitators recognise their privileged position as stakeholders who, despite striving to leave bias and subjectivity on one side, will nevertheless have personal objectives and motives for becoming involved. This will help facilitators to be more selfaware and protect against undue bias.

\subsection{Data gathering and review}

\subsubsection{Data requirements}

The principal aim of data gathering is to prepare an overall description of the protected area, including an inventory of the known biodiversity components that are present. This should be carried out in partnership with stakeholders, by collating and reviewing all relevant and available information on the protected area's status, biophysical characters, human use and biodiversity. Additional information may also need to be gathered from new field surveys and analysis of remote sensing data.

This stage of an assessment may potentially be very time consuming, and could easily become overwhelming, so it is important to focus attention on key information requirements of the stakeholders that are directly relevant to the management planning process (see Box 3.1). It is often best to collect the minimum of information first and then identify other requirements as other stages of the management plan progress. This helps to ensure that all information collected is relevant and avoids wasting time whilst irrelevant descriptive information is compiled.

Information requirements for a protected area management plan assessment typically include:

- Location and boundaries, and appropriate administrative boundaries (mapped).

area.

- Status (e.g. international, national and local designations and IUCN protected area category).

a Administration (e.g. with respect to protected area, forestry, water resources and community management). 
- Land ownership and occupancy.

- Infrastructure and services (e.g. roads, airports, telecommunications, power supplies).

a Physical information (e.g. geology, soils, topography, climate, hydrology).

- Land use (historical and current).

- Cultural information (cultural values and interactions with landscape and biodiversity).

- Socio-economic status and trends, and relationship with the protected area and its features.

- Visitor numbers, interests and influences.

- Ecosystems and habitats, (including the types of ecosystem and habitat that are present, with maps of their location, quantification of their current and past extent and condition, and descriptions of their use and management by local communities).

- Important flora and fauna, including protected species, threatened species, utilised wild species, ecological keystone species and species of cultural importance. And for each species information on:

- Quantity: population sizes, abundance, stock volume, basal area.

- Quality: importance, trends in abundance, productivity and viability.

- Location: distribution; relationship between place and cultural value.

- Value: use by humans (e.g. food, forage for livestock, materials, medical uses, cultural uses), trends in uses, conservation importance, aesthetic values etc.

- Domestic livestock, pest species and introduced species (listed and their interactions with native species and ecosystems described).

- Factors affecting habitats and important flora and fauna, including pressures and management responses.

\subsubsection{Information sources}

\section{Existing information}

Some of this information may be obtainable from scientific books, papers and reports, land-use and habitat maps, aerial photographs, satellite images, historical records and unpublished data held by experts. However, it will normally be essential to supplement such scientific data with information from local people, gained through participatory involvement in the assessment process where possible.

Any information and documents on the reasons for the establishment of the protected area and the definition of its boundaries will be a useful start. Records held by government agencies and religious authorities of land tenure, population census, tax collection, and agricultural, forestry and fisheries production are all valuable resources.

\section{New surveys}

In some cases it may be necessary to collect new data to prepare biodiversity assessments that are adequate for management plans. However, incomplete information should not be used as an excuse for delaying management planning. In many cases it will be possible to 
complete management plans with available data and to include further surveys amongst the agreed actions, the results of which then feed back into the plan, thus completing the adaptive planning loop.

Where surveys are required, these may necessitate fieldwork to establish species presence, quantify species populations, map habitats and assess habitat conditions. These may be carried out using some of the methods described in Chapter 3 of these guidelines, or as described in Sutherland (2000) and Hill et al. (in press). Local people may be able to undertake some surveys (with any necessary guidance and training), thereby further enhancing the participatory process. If surveys must be carried out by outside experts then suitable local people (e.g. with an interest in wildlife or resource use) should be invited to take part, perhaps as trainees or apprentices so that local capacity is developed to carry out future surveys.

\section{Remote sensing data}

Habitat surveys may be supported using remote sensing data and Geographical Information Systems (GIS). A GIS is a spatially referenced database that allows multiple layers of data to be created and displayed together as computerised maps. Data sources may include satellite data, aerial survey, existing maps, field survey and expert knowledge. GIS enables the standard formatting of all maps used, no matter what their source. For further background information on GIS, see Longley et al. 2001, and Burrough \& McDonnell, 1998.

Both satellite scenes and aerial photographs are types of remotely sensed data. The main advantages of satellite data are that large and inaccessible areas can be covered using a standard approach, with a uniform level of detail and at relatively low cost. The use of such data may therefore be particularly cost-effective for mountainous areas, such as the ACA, with their extremely difficult terrain, with the caveat that remotely sensed data are not useful for areas that are persistently obscured by cloud. Remotely sensed data may be extremely valuable for monitoring as repeated surveys using identical techniques are possible throughout the lifetime of the remote measurement system (an anticipated 15 years for the MODIS instruments on board EOS satellites). The maps produced can be used alongside other layers in a GIS for land cover mapping, modelling and planning. However, a specialised set of skills and software are required to interpret raw remotely sensed data - it should not be assumed that all GIS software and personnel can carry out image analysis.

Two sources of remotely sensed data are the LANDSAT and EOS satellites. The first LANDSAT satellite was launched in 1972, and the most recent in 1999, followed by the EOS satellite in 2000. LANDSAT covers the Earth in 18 days and has a pixel resolution of $30 \mathrm{~m}$. The MODIS Terra sensor on the EOS satellite visits every location every 1 to 2 days, and has a pixel resolution of $250 \mathrm{~m}$. LANDSAT is therefore better for monitoring fine resolution, small area or relatively slow changes (such as changes in forest cover), and MODIS for coarser resolution, large area or relatively fast changes (such as fires).

Free LANDSAT and MODIS satellite images can be readily obtained from the internet. These can be found for a particular area using the USGS 'GloVis' interactive map (http:// glovis.usgs.gov/). Once the path and row number of the image(s) of interest have been found, the Earth Science Data Interface site (http://glcfapp.umiacs.umd.edu:8080/esdi/index.jsp) can be used to seek free images. The main scene for the Annapuma area has path 142 and row 40 . 
Aerial photographs can be used to examine smaller areas at a finer spatial resolution, and to support the interpretation of satellite data. Vegetation types are most effectively identified in satellite images when the classification is 'supervised'. This requires a training set, used to inform the software about different land cover on the ground. Satellite data therefore are of most use when brought together with existing maps or georeferenced aerial photographs.

Habitat maps may be used in the design of a stratified sampling regime for species or community assessment. The habitat maps can also be used to model species" distributions or abundance. These models may either directly be used to estimate species occurrence or to identify areas for sampling. A number of techniques and packages for species distribution modelling are listed in Table 4.8 (Section 4.15).

A unique contribution that can be made by satellite data is information on habitat changes prior to the commencement of the assessment and monitoring programne. A change analysis (comparison between years) highlights areas where land cover change has occurred. These may be areas in need of conservation attention, or conversely where ecosystem restoration is in progress.

\subsubsection{Data presentation}

Presentation of the descriptive information in the management plan should be brief and easily understandable, and should focus on the key results of the assessment. Full use should be made of maps and diagrams, with attention to design so that the most important information is easily visible. Information that is not directly required should either be referred to (with full reference details) or placed in a separate technical appendix. Technical language and jargon should be avoided where possible, and a glossary provided for all technical terms that are used. Local names of animals and plants should be given as well as scientific names.

\subsection{Biodiversity evaluation}

\subsubsection{The purpose of a biodiversity evaluation}

In general terms biodiversity evaluation ${ }^{2}$ is the process of establishing the value (ideally quantitatively) of biodiversity components. Evaluations may be carried out on various components of biodiversity (i.e. from genetic variation within species, to individual species, species assemblages, habitats, sites and biomes).

In the context of protected area management planning, the overall purpose of a biodiversity evaluation is to establish why the protected area is important and of value to society (and hence protected) and what are its key features (and their values) that need to be conserved (i.e. protected and/or used sustainably). It is normally carried out to identify sites that merit some form of protection, but is often repeated and updated as part of management planning process (especially when the reasons for creating a protected area have been illdefined or poorly communicated).

:Biodiversity evaluation should not be confused with monitoring cialuation (or result cvaluation or management evaluation or similar terms), which is the measuring of progress with respect to management objectis es. 
Biodiversity evaluation is fundamental to any management planning process because it identifies the features (such as particular habitats, threatened species, and medicinal plants) that should be the focus of management actions. Unless protected area values are understood, there is a risk that inappropriate management may be undertaken, resulting in a decline in the value of the protected area and its important features. The evaluation, therefore, forms the basis upon which conservation objectives should be set, which explicitly ensure that each feature is conserved appropriately (see Section 3.5).

Great care must, therefore, be taken in the evaluation of the biodiversity assessment data. And it is particularly important that all stakeholders participate in the evaluation process, to ensure that all biodiversity values are identified. "Unless the preparation of the Management Plan addresses the concerns of the local people then their support will be impossible to secure" (Thomas and Middleton 2003). Local people, if given the opportunity to discuss findings, can also often provide interpretations and insights that otherwise may have been missed if the results had been interpreted solely by staff and advisors (Steinmetz 2000). Moreover, the drafted solutions emerging from participatory data analysis will be more practical and adjusted to the local conditions.

\section{A biodiversity evaluation consists of two parts:}

- An evaluation of protected area as a whole, which places the site in the context of its regional, national and international importance, and identifies its overall value and importance to various interest groups.

- The identification of the key biodiversity features that must be protected and conserved to maintain the importance of the protected area.

\subsubsection{Carrying out an overall biodiversity evaluation of the protected area}

An evaluation of the biodiversity of a protected area as a whole may take into account a wide range of potential biodiversity values, including intrinsic and socio-cultural values (Daily 1997, Posey 2000), and more direct socio-economic benefits (Daily 1997), such as food, building resources, medicines and waste decomposition etc (Spellerberg and Hardes 1992).

The assessment of the ecological values (many of which are used for protected area selection) is often the focus of nature conservation management plans, and various criteria have been developed for these evaluations. Although, no standard set of criteria have emerged for this purpose, one set that have been frequently used are those developed by Ratcliffe (1977), as listed below:

\section{Primary criteria}

- Size (including the protected area and habitats, and population sizes for key species).

- Diversity (of habitats and species).

- Rarity (of habitats and species).

- Naturalness (habitats that are least modified by human use tend to hold richer wildlife communities, often including rare species, and have high aesthetic appeal and scientific value).

- Typicalness (how well the area represents ecosystems and habitats on a wider scale). 
- Fragility (how vulnerable or sensitive ecosystems, habitats and species are to human induced changes).

\section{Secondary criteria (i.e. not always used)}

- Recorded history (sites that have been studied and valued for a long time tend to be highly valued).

- Potential value (i.e. the likelihood that appropriate management could restore or enhance an area's ecological value).

- Position in geographical or ecological unit (e.g. some ecosystems, habitats and species may be of high functional importance).

- Intrinsic appeal (e.g. species with public appeal promote the cause of nature conservation and act as flagship species).

These criteria were first used in the United Kingdom to identify sites of high conservation value, which later formed the basis for identifying Sites of Special Scientific Interest (Nature Conservancy Council 1989). These criteria have been widely adopted and adapted in the UK for management planning evaluations, e.g. by the RSPB (Hirons et al. 1995) and the Countryside Council for Wales (CCW 1996). Although developed in the UK, they are easily applicable to other situations and have been widely adapted for use in many other countries, e.g. in the Ramsar Management Planning Guidelines (Ramsar Bureau 2002)

Evaluations are simply carried out by considering the properties of the protected area in relation to each of the criteria in turn. This may then be summarised in a description of the overall importance of the protected area, such as the example for the ACA in Box 3.2.

\section{Box 3.2. A possible ecological evaluation summary statement for the Annapurna Conservation Area}

The ACA is a very large $\left(7,629 \mathrm{~km}^{2}\right)$ area of Himalayan mountain ecosystem, which overlaps several biogeographical regions and holds a diverse range of habitats and species. The Kali Gandaki Valley runs through the ACA and is a biogeographical divide in the Himalayan mountain chain. The ACA therefore has species typical of the eastern and western Himalayas. The Kali Gandaki valley is also an important north/south bird migration route. The ACA also lies within a region of overlap between the Palaearctic and Indo-Malayan realms and so has species of both realms. The ACA has a huge altitudinal range, from 790 to $8,091 \mathrm{~m}$. It has a great range of rainfall, with some of the driest regions in Nepal in the trans-Himalayan zone and some of the wettest regions south of Annapurna in the Modi Khola valley. Although some of the habitats may not be species rich, they hold important populations of several globally threatened species, some of which are of exceptional intrinsic appeal (e.g. Snow Leopard).

However, many management plans fail to properly identify other values of the biodiversity of a protected area, such as cultural, social and economic values (Thomas and Middleton 2003). This is unwise as it may undermine the potential support of local people for the protected area and its management. 


\subsubsection{Identifying key biodiversity features of a protected area}

Key biodiversity features (e.g. species, habitats, ecological functions) should include features that are:

- of high nature conservation importance.

- socio-economic importance.

- cultural importance.

The identification of key biodiversity features of high nature conservation importance should firstly take into account broad international and national conservation objectives. In terms of global objectives, there is broad agreement that the prevention of global extinction should be the highest priority, and therefore the degree of threat (i.e. risk of extinction) is of primary concern in setting priorities. This is reflected in the production of IUCN Red Lists (see www.redlist.org) of species that are considered to be at risk of global extinction according to various categories of threat. The risk of extinction at national level is also the commonest basis for identifying national species conservation priorities.

Rarity has often been considered to be one of the most important factors influencing the risk of extinction of a species, and many Red Lists have focused on this. Rarity has also often been used as a secondary criterion whereby, for example, a declining species is not considered to be threatened unless it has also crossed a rarity threshold. However, rarity is not a straightforward concept as there may be a variety of circumstances under which species may be rare (Rabinowitz 1981). Species may have small (or large) total ranges, occupy few (or many) habitat types, and may be scarce or abundant where they do occur. It is evident that rarity includes both a spatial and a numerical dimension. For some species some aspects of rarity may be an evolutionary property, as a result of their habitat specificity, small natural range or low natural densities. On the other hand, small range or low densities may be the result of human impact, which may be reversible.

Consideration of rarity in an evaluation requires data on the range or number of individuals of a species (or habitats, communities, or abiotic features), not only within the protected area but at wider scales. This is because important elements of rarity are scale dependent. For example, a locally rare species may also be regionally or globally rare, which is of greater importance than if it is only locally rare because it is at the edge of its range (e.g. Himalayan Musk Deer Moschus chrysogaster, or Snow leopard Uncia uncial or Golden eagle Aquila chrysaetos). Normally, increased importance to rarity should be given to species that are rare at the global level. Some locally common species may also be of high conservation importance if the species in question is rare at a global or wide geographical scale (e.g. Blue Sheep Pseudois nayaur in the ACA).

Whichever criteria are used for evaluations, a hierarchical level of importance should be established so that the highest priority for conservation/protection is given to species, or habitats, that are globally threatened. But it is also necessary to take into account their local status to assess the necessity for taking action at a local scale. This enables the principle of "thinking globally and acting locally" to be put into practice. The highest priority should be given to species and habitats that are both globally and locally threatened. 
Assessments below global scales should be based on appropriate biogeographical populations where possible. In practice though, assessments of populations are more often based on national or regional populations for political and administrative reasons (e.g. as in most national Red Lists).

Any evaluation of conservation priorities for a species or habitat should also take into account the importance of the population or resource being considered. Thus, the evaluation of a habitat area or a species' population should consider two key independent factors:

- the risk of loss of the habitat or species population (i.e. its threat status).

- its biogeographical importance, i.e. the proportion of the biogeographical (or national/ regional) resource or population.

Thus, for example, a very high priority should be given to a species' population that is biogeographically important (i.e. it is a large proportion of the species' entire population) and at risk of extinction within the area in question. However, it is important to note that a species population may be a high priority nationally because it is highly threatened nationally, irrespective of its numbers in relation to international or global populations e.g. Himalayan Musk Deer in Nepal. This is because the maintenance of a species' range (and potential genetic variation associated with this) can also be an important conservation aim after prevention of complete extinction. On the other hand. a population of a species may be very important because it is a large proportion of the biogeographical population, irrespective of its conservation status, e.g. Spiny Babbler Turdoides nipalensis, a bird species which is not uncommon in secondary scrub in the ACA, but is endemic to Nepal. In these circumstances a country has a particular responsibility for the species and should at least monitor the status of the species and guard against potential events that could impact on the population.

Taking into account the issues described in this section and 3.3.2. it is possible to suggest a checklist (Table 3.1) of biodiversity features that should be considered as key features, or features of exceptional value, according to the IUCN terminology (Thomas and Middleton 2002).

\section{Table 3.1. Biodiversity components that should be considered to be key biodiversity features within a protected area.}

Globally threatened species (i.e. on IUCN Red Lists)

Significant populations of rare or otherwise nationally or regionally threatened species

Endemic species or other species that have very high proportions of their biogeographic or global populations within the protected area or region

Rare or threatened habitats (globally, regionally or nationally)

Particularly good examples (e.g. large and highly natural) of characteristic habitats

Features of high intrinsic ecological importance or interest

Features of high socio-economic importance (e.g. forest products, grazing lands or species and habitats that attract tourists)

Features of high cultural importance (e.g. sacred areas or species) or intrinsic appeal 
In practice it is often impractical to list and set objectives for all the habitats and species that may qualify as key features. Some key features may therefore be combined. For example a number of important medicinal plants may be just listed as medicinal plants of temperate broad-leaved forests.

It is good practice at this stage not to include habitats or species etc as key features if they are only of indirect importance (e.g. as a habitat or prey species) for other listed key features. Key features need to be important in their own right. This can be tested by considering if a substantial decline in the feature in the protected area would be unacceptable if all other features were maintained (irrespective of how unlikely this might be). Thus for example, a decline in Blue Sheep populations in the ACA would be extremely undesirable, not just because it is a prime prey species for Snow Leopard. Thus this qualifies as a key biodiversity feature. In contrast, some other key prey species might not be listed as key features. This does not mean that management actions and monitoring may not be undertaken for such prey species, but these requirements would be identified at a later stage of the management planning.

It is also advisable to avoid listing species as key features at this stage if they only act as indicators (see Box 3.3) of a feature's value, for example, a tree species as an indicator of diverse broad-leaved forests. In this case the feature would be "diverse broad-leaved forest". If it is necessary to use indicator species to define conservation objectives for this, these should be identified at the conservation objective setting stage. However, it may be useful to note that a species may sometimes act as an indicator of one feature and be a key feature in its own right.

For further information on biodiversity evaluations see Margules and Usher (1981), Smith and Theberge (1986), Usher (1986), and Spellerberg (1992).

\section{Key biodiversity features within the Annapurna Conservation Area}

On the basis of the criteria listed in Table 3.1, it is possible to provide a preliminary list of some habitats and species that should be considered to be key biodiversity features within the ACA (see Table 3.2). 
Table 3.2. Some key biodiversity features within the Annapurna Conservation Area

\begin{tabular}{|c|c|}
\hline Key Feature & Rationale for listing as key feature \\
\hline $\begin{array}{l}\text { Caragana, Lonicera, Carex and Cobressia } \\
\text { species grasslands of the trans-Himalayan } \\
\text { Rangelands (Mustang and Manang) }\end{array}$ & $\begin{array}{l}\text { Characteristic habitat of Trans-Himalayan } \\
\text { region, of key importance for several } \\
\text { threatened species, high socio-economic } \\
\text { importance. }\end{array}$ \\
\hline $\begin{array}{l}\text { Singdi forest, Pasgaon Village Development } \\
\text { Committee, Lamjung }\end{array}$ & $\begin{array}{l}\text { Rare forest type and biodiversity hotspot } \\
\text { (supports over } 250 \text { plant species, also } \\
\text { recognized by ACA Management Plan, 1997), } \\
\text { has also patch of rare Hill Sal (Shorea robusta) } \\
\text { forest. } \\
\text { High socio-economic importance }\end{array}$ \\
\hline $\begin{array}{l}\text { Taxus baccata forest, primarily in Manang and } \\
\text { Mustang (below Larjung) }\end{array}$ & $\begin{array}{l}\text { High commercial demand } \\
\text { High socio-economic importance }\end{array}$ \\
\hline $\begin{array}{l}\text { Pure Birch Betula spp. forest in gorge near } \\
\text { Ngawal (northern side of Ngawal) }\end{array}$ & $\begin{array}{l}\text { Rare forest type, only known pure forest of } \\
\text { Birch in ACA (KMTNC, 1994); socio- } \\
\text { culturally important }\end{array}$ \\
\hline $\begin{array}{l}\text { Hill Sal (Shorea robusta) forest, Madi, Khudi } \\
\text { and Rudi Basin }\end{array}$ & $\begin{array}{l}\text { Sal is a typical high value tropical species, rare } \\
\text { in ACA, High socio-economic importance; a } \\
\text { suitable habitat for Clouded Leopard }\end{array}$ \\
\hline $\begin{array}{l}\text { Juniperus forest stands of Samar, Upper } \\
\text { Mustang }\end{array}$ & $\begin{array}{l}\text { Last remaining area of forest in upper } \\
\text { Mustang, culturally and ecologically important }\end{array}$ \\
\hline Rhododendron forest of Ghandruk-Ghorepani & $\begin{array}{l}\text { High aesthetic appeal, and one of the largest } \\
\text { pure such forest in Nepal, with National flower } \\
\text { (Rhododendron arboreum) }\end{array}$ \\
\hline Forests in the intensive use zone & $\begin{array}{l}\text { High socio-economic importance: fuelwood, } \\
\text { timber and non-timber products }\end{array}$ \\
\hline $\begin{array}{l}\text { Major non-timber forest products: Daphne } \\
\text { spp., Swertia chiraita (Chiraito), Piper longum, } \\
\text { Arundinaria spp., Alliums spp., Swertia spp. } \\
\text { (found in upper Mustang), Aconitum spicatum } \\
\text { (Bikh), Cordyceps sinensis (in Manang), } \\
\text { Dactylorhiza hatagirea (Panch Aule), } \\
\text { Picrorhiza scrophulariflora (Kutki), Paris } \\
\text { poliphyla (Satuwa) }\end{array}$ & $\begin{array}{l}\text { High socio-economic importance and some } \\
\text { species protected in Nepal }\end{array}$ \\
\hline Snow Leopard Uncia uncia & $\begin{array}{l}\text { Globally threatened: Endangered, protected in } \\
\text { Nepal, CITES I, species of very high intrinsic } \\
\text { appeal. }\end{array}$ \\
\hline Clouded Leopard Neofelis nebulosa & $\begin{array}{l}\text { Globally threatened: Vulnerable, protected in } \\
\text { Nepal, CITES I } \\
\text { (Cont.) }\end{array}$ \\
\hline
\end{tabular}

Note: Global threats status is based on 2004 IUCN Red List of Threatened Species (IUCN 2004), unless otherwise indicated. 
Table 3.2. Some key biodiversity features within the Annapurna Conservation Area (cont.)

\begin{tabular}{|c|c|}
\hline Asiatic Wild Dog Canis alpinus & Globally threatened: Endangered \\
\hline Red Panda Ailurus fulgens & $\begin{array}{l}\text { Globally threatened: Endangered, protected in } \\
\text { Nepal, CITES I }\end{array}$ \\
\hline Assamese Macaque Macaca assamensis & $\begin{array}{l}\text { Globally threatened: Vulnerable and protected } \\
\text { in Nepal }\end{array}$ \\
\hline Asian Wild Ass Equus hemionus & Globally threatened: Vulnerable \\
\hline Himalayan Musk Deer Moschus chrysogaster & $\begin{array}{l}\text { Globally Near Threatened, CITES I, protected } \\
\text { in Nepal }\end{array}$ \\
\hline Himalayan Tahr Hemitragus jemlahicus & Globally threatened: Vulnerable \\
\hline Tibetan Antelope Pantholops hodgsonii & $\begin{array}{l}\text { Globally Threatened: Endangered, CITES I, } \\
\text { protected in Nepal }\end{array}$ \\
\hline Argali Ovis ammon & $\begin{array}{l}\text { Globally Threatened: Vulnerable, protected in } \\
\text { Nepal, CITES I }\end{array}$ \\
\hline Wood Snipe Gallinago nemoricola & $\begin{array}{l}\text { Globally threatened: Vulnerable (BirdLife } \\
\text { International 2003); species of high meadows }\end{array}$ \\
\hline $\begin{array}{l}\text { Slender-billed Vulture Gyps tenuirostris, } \\
\text { Oriental White-backed Vulture Gyps } \\
\text { bengalensis and Himalayan Griffon Vulture } \\
\text { Gyps himalayensis, }\end{array}$ & $\begin{array}{l}\text { Slender-billed and Oriental White-backed } \\
\text { Vultures are Globally threatened: Critical } \\
\text { (BirdLife Intemational 2003), Himalayan } \\
\text { Griffon is common, but may be declining. }\end{array}$ \\
\hline Cheer Pheasant Catreus wallichii & $\begin{array}{l}\text { Cheer Pheasant is globally threatened: } \\
\text { Vulnerable (BirdLife International 2003) and } \\
\text { protected in Nepal }\end{array}$ \\
\hline
\end{tabular}

\subsection{Identification of constraints, opportunities and pressures}

\subsubsection{The purpose of identifying constraints, opportunities and pressures}

This stage of management planning aims to identify potential constraints, opportunities and pressures that may influence the development of the overall vision for the protected area and specific management objectives. For example, constraints may include the legal and institutional framework for the protected area, and resource, communication and infrastructure limitations. Opportunities might include the potential capacity for increasing revenue from tourism, new technological developments (e.g. micro-hydropower) or activities that may bring unintended biodiversity benefits (e.g. afforestation programmes for soil stabilisation).

The identification of pressures that may threaten biodiversity is particularly important and the aim should be to comprehensively identify all the pressures on the key features of biodiversity importance. Where possible, these pressures should be quantified. Risk assessments should be carried out for features of high importance, which take into account both the probability of impacts and the likely magnitude of those impacts. 
It is useful to develop a simple framework of the interrelations between important biodiversity features and their influencing factors and management actions. This helps to understand what management actions need to be taken and what needs to be monitored. A widely used framework which is particularly useful in such assessments is the Pressure-State-Response framewr. $^{3}$ (see Figure 3.2). This was designed to aid analysis of the causes of change in the natural environment and the response measures of human society to these changes.

\section{Figure 3.2. The Pressure - State - Response framework}

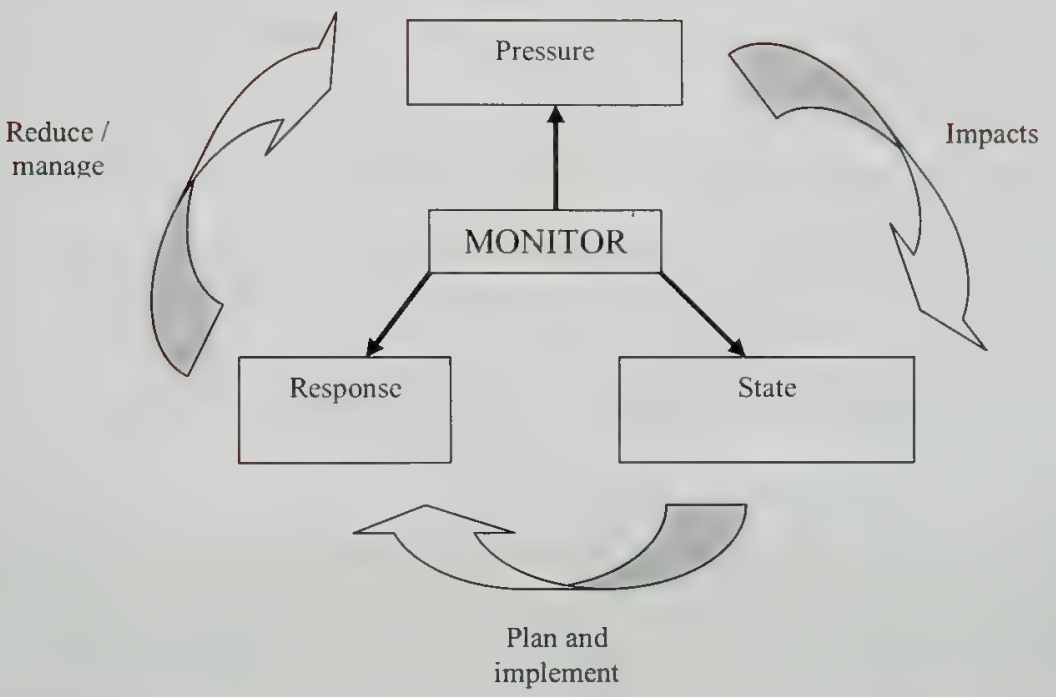

When applied to a protected area, the State component is an assessment of the current state or condition of a biodiversity feature in the area, and of the changes that it has undergone in the past. This might focus on, for example, the extent and condition of important forest habitats within the protected area.

The Pressure component is an assessment of what factors may potentially impact on the biodiversity feature that influences its state. Examples of such pressures on the state of important forest habitats could be deforestation for firewood and timber, pollution, or hunting.

The Response component is an assessment of the policies, laws, practices, etc. that have been created to manage and conserve the biodiversity feature and alleviate or regulate the pressures on it. The designation as a protected area is one response in itself, whilst others could be tree planting programmes, awareness activities and hunting regulations.

${ }^{3}$ This framework may sometimes vary, for example as Driving Force - State - Response, or Driving Force Pressure - State - Response, or other more complex variations 


\subsubsection{Identifying pressures on key biodiversity features}

This stage of the assessment requires a review of available relevant information. Such information, will include studies of the protected area itself. Some of this may be published, but much may also be learnt from consultations with local experts. Published scientific studies of other protected areas and the knowledge gained by protected area managers and scientists elsewhere may also be relevant and useful. However, participatory approaches to assessments of pressures on biodiversity may be particularly valuable and often the only source of information in many areas. Local people may be able to provide particularly useful information, which may be unavailable from other sources, especially regarding exploited biodiversity components (e.g. forest products, hunted species and rangelands). However, it is important to be aware that some opinions relating to possible pressures on important socio-economic assets, such as the impacts of predators on livestock populations, may be misconceptions or exaggerated by some stakeholders.

\section{Pressures on key biodiversity features within the Annapurna Conservation Area}

Using this framework we can summarise the state and pressure of some key features in the ACA according to available information as indicated in Table 3.3.

\section{Plate 3.2 Fuel wood collection from forest}

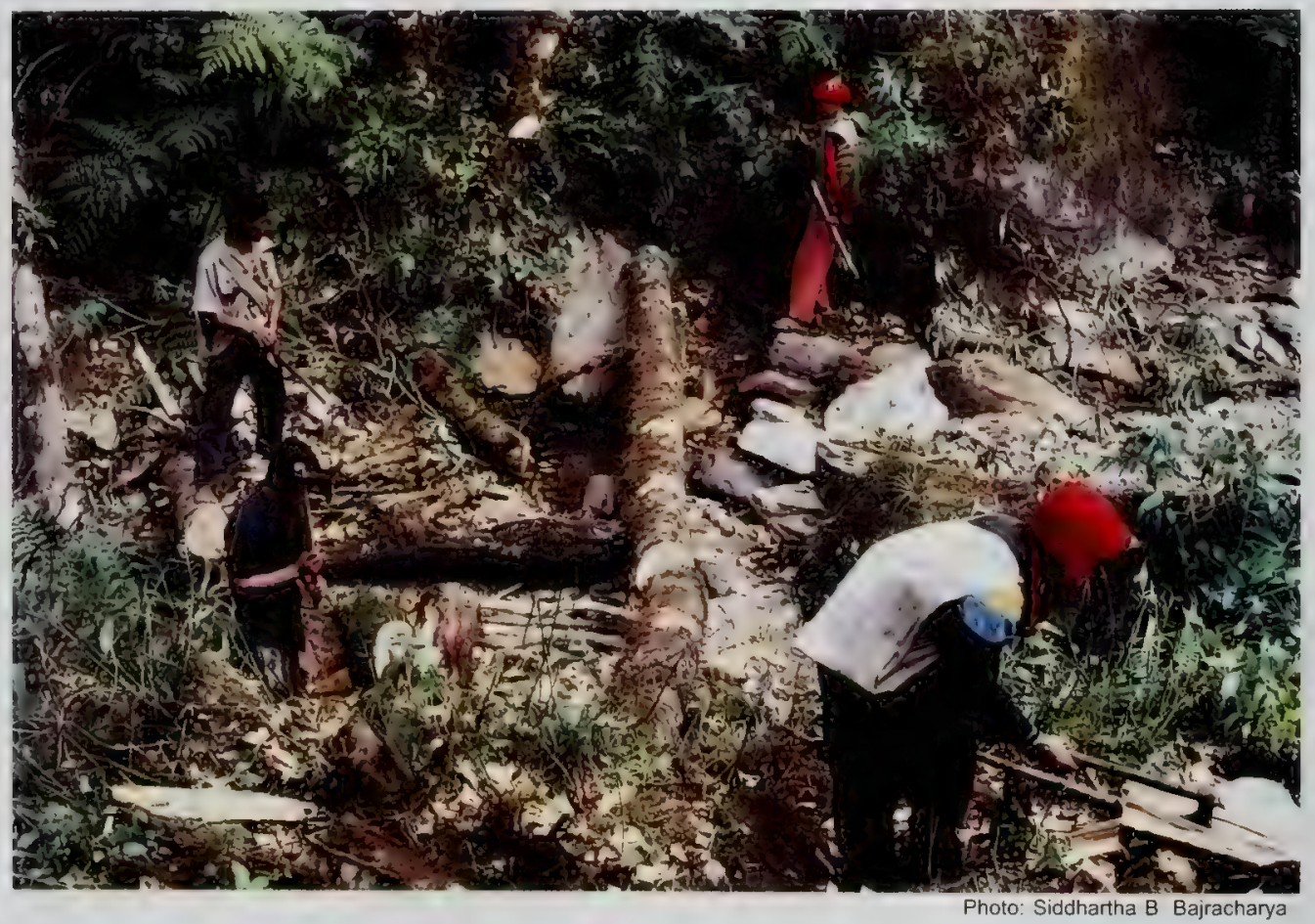


Table 3.3. The state of some key biodiversity features within the Annapurna Conservation Area and pressures affecting them

\begin{tabular}{|c|c|c|}
\hline Key feature & State of feature & $\begin{array}{l}\text { Potential pressures on } \\
\text { feature }\end{array}$ \\
\hline $\begin{array}{l}\text { Caragana, Lonicera, Carex and } \\
\text { Cobressia species grasslands of the } \\
\text { trans-Himalayan Rangelands } \\
\text { (Mustang and Manang) }\end{array}$ & $\begin{array}{l}\text { Degraded in over-grazed } \\
\text { areas }\end{array}$ & $\begin{array}{l}\text { Livestock grazing pressure, } \\
\text { closure of Tibetan rangelands for } \\
\text { grazing Nepalese livestock }\end{array}$ \\
\hline $\begin{array}{l}\text { Juniperus forest stands of Samar, } \\
\text { Upper Mustang }\end{array}$ & Very sparse trees & $\begin{array}{l}\text { Fuelwood collection (lucrative } \\
\text { source of fuelwood compared to } \\
\text { its alternative of Caragana) }\end{array}$ \\
\hline $\begin{array}{l}\text { Rhododendron forest of Ghandruk- } \\
\text { Ghorepani }\end{array}$ & Degrading in certain parts & $\begin{array}{l}\text { Tourism with increased demands } \\
\text { for fuelwood and timber. }\end{array}$ \\
\hline Major non-timber forest products & $\begin{array}{l}\text { Increasing stock in some } \\
\text { area whereas decreasing in } \\
\text { the areas where illegal } \\
\text { collectors harvest } \\
\text { unscientifically }\end{array}$ & $\begin{array}{l}\text { Illegal harvesting and higher } \\
\text { commercial demand for legal } \\
\text { harvesting }\end{array}$ \\
\hline Snow Leopard Uncia uncia & $\begin{array}{l}\text { Inadequate information on } \\
\text { nopulation. }\end{array}$ & $\begin{array}{l}\text { Retaliatory killing by herders and } \\
\text { poaching. }\end{array}$ \\
\hline $\begin{array}{l}\text { Himalayan Musk Deer Moschus } \\
\text { chrysogaster }\end{array}$ & $\begin{array}{l}\text { Lack of detailed infor- } \\
\text { mation on population. } \\
\text { Increasing compared to } \\
1-2 \text { decades ago according } \\
\text { to local people }\end{array}$ & $\begin{array}{l}\text { High commercial interest leading } \\
\text { to poaching and disturbances of } \\
\text { habitat. }\end{array}$ \\
\hline $\begin{array}{l}\text { Rare Tibetan Plateau species - } \\
\text { Asian Wild Ass, Tibetan Antelope, } \\
\text { Argali }\end{array}$ & $\begin{array}{l}\text { Little known. First } \\
\text { scientific records in } 2001 .\end{array}$ & $\begin{array}{l}\text { Competition with livestock for } \\
\text { grazing. Possible hunting. }\end{array}$ \\
\hline Wood Snipe Gallinago nemoricola & Rare, trends uncertain & Uncertain, possible overgrazing. \\
\hline $\begin{array}{l}\text { Slender-billed Vulture Gyps } \\
\text { tenuirostris, Oriental White- } \\
\text { backed Vulture Gyps bengalensis } \\
\text { and Himalayan Griffon Vulture } \\
\text { Gyps himalayensis, }\end{array}$ & $\begin{array}{l}\text { Substantial declines in } \\
\text { Slender-billed Vulture and } \\
\text { White-rumped Vulture and } \\
\text { some evidence of smaller } \\
\text { declines in Himalayan } \\
\text { Griffon Vulture. }\end{array}$ & $\begin{array}{l}\text { Possible poisoning from } \\
\text { Diclofenac, a non-steroidal anti- } \\
\text { inflammatory drug used for } \\
\text { livestock, as across much of the } \\
\text { Indian subcontinent (Oaks et al. } \\
\text { 2004, Shultz et al. 2004). }\end{array}$ \\
\hline Cheer Pheasant Catreus wallichii & Rare, trends uncertain & $\begin{array}{l}\text { Livestock grazing, forest fires, } \\
\text { hunting. }\end{array}$ \\
\hline
\end{tabular}

It may be seen from this table that some pressures are common to more than one feature (e.g. over grazing may impact on some wild threatened species and livestock) and some pressures may be related to the state of some features. For example, degradation of the State of the trans-Himalayan grasslands may result in a pressure on Snow Leopards as a result of indirect impacts on their prey. 
Pressures may therefore be interrelated and complex, as indicated in Figure 3.3, which summarises the example of pressures that are thought to affect Snow Leopards in the ACA, based on studies within the ACA (Oli, 1991; Jackson et al. 1996; Thapa 2000), and elsewhere (Jackson et. al 2001).

\section{Figure 3.3 An illustrative example of the pressures affecting Snow Leopards in the ACA.}

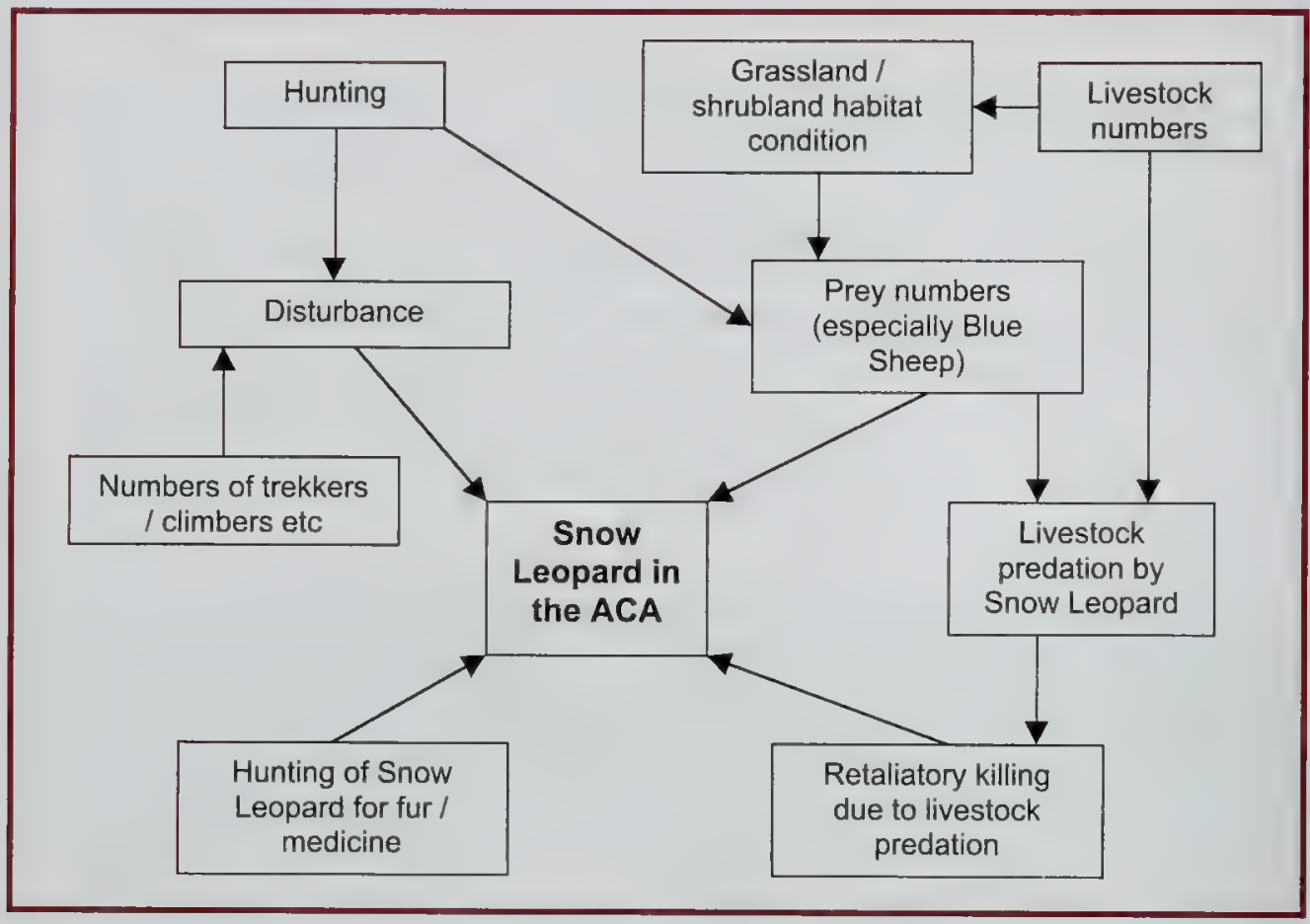

\subsection{From the assessment to setting objectives}

\subsubsection{Vision, goals and objectives in protected area management}

Once the biodiversity assessment has been conducted the results should be analysed and communicated to the planners and managers of the area. The assessment forms the basis for developing a long-term vision, broad goals and specific objectives within the management planning process (see Figure 2.2 and 3.1). A vision statement aims to provide a broad and inspirational description of the desired future of the protected area. Its main purpose is to provide a focus or direction for management objectives and, according to Thomas and Middleton (2003), should:

- Describe the kind of protected area that the plan is seeking to achieve in the long term. This will help people to understand what it is hoped the area will be like in the future, the reasons for this, and the action needed to achieve the vision.

a Be a long-term statement that is unlikely to change significantly over time. It should therefore provide continuity in the process of managing the protected area in a sustainable way. 
- Include environmental, recreational, cultural and social and economic aspects of the protected area.

The vision should be developed in a participatory manner, ideally with all key stakeholders, to ensure that all interests are adequately dealt with and that there is broad ownership of the vision.

Broad goals (or aims) relate to particular features or functions of the protected area, and indicate general principles and directions of change. For example, the KMTNC 1997 ACA Management Plan lists the following three primary long-term goals:

a to conserve the natural resources of the ACA for the benefits of the present and future generations;

- to bring sustainable social and economic development to the local people; and

a to develop tourism in such a way that it will have a minimum negative environmental impact.

Objectives are specific outcomes or targets that the management activities will be designed to achieve. Objectives should be clear descriptions of a measurable standard to be achieved, or a desired state, threshold value, amount of change, or trend that you are seeking to establish. Such objectives are often referred to as being SMART, i.e.:

Specific: Objectives must be focused and precise so that all stakeholders have a consistent understanding of what is planned. For example, an objective such as "to conserve threatened wildlife" would mean many different things to different people. Thus objectives should not be easy to misinterpret. It is easier to identify and plan required actions if objectives are specific.

Measurable: It is vital to be able to clearly determine whether or not an objective has been reached. This can be done if measurable units are used to define the objective. it may be relatively straightforward to set measurable objectives for habitat quantity or individual species (e.g. a specific population size), but it is difficult to measure some objectives, suck as those relating to habitat quality. Biodiversity indicators may therefore be selected to enable measurable targets to be set (see Box 3.4). For example, forest habitat quality might be indicated by the presence of particular tree species that are only ever found in good quality forests (i.e. old growth native forests that have not been subject to significant exploitation). A measurable definition of forest quality may then be defined by setting a minimum frequency of occurrence or density of the indicators.

Achievable: It must be possible to achieve the objectives within the protected area. For example, it would not be possible to ensure the maintenance of a declining migratory bird population (as it might be declining due to impacts outside the protected area). But it would be appropriate to set an objective for its conservation within the protected area (c.g. related to maintaining breeding success).

Realistic: Objectives should not be aspirational. such as to reverse all previous forest loss. This might be a suitable long-term vision, but a more suitable objective for a management plan might be to reverse $20 \%$ of previous loss over the 10 year period of the management plan. 
Time-specific: It is important to set a time period for reaching the objective, to help prioritise and plan actions. For maintenance objectives where ongoing actions are required, the objective should state the period over which the objective will apply.

The rationale for objectives should be clearly explained within management plans. Some form of priority category should also be given to each objective, so that decisions can be made without re-evaluations if resource or time limitations require some objectives to be dropped. Secondary objectives may also be included in case resources increase unexpectedly. Objectives should be set for the biodiversity features themselves (i.e. their state) the factors that influence them (i.e. pressures) and for the management activities that may influence these pressures (i.e. the responses). Each of these objectives should then be monitored (see Figure 4.1).

\subsubsection{Setting objectives for the state of key biodiversity features}

The setting of objectives for a biodiversity feature (or group of features) involves the definition of the following six components:

1. Selection of the key biodiversity features within the protected area that will be the focus of management and monitoring (see 3.4 .3 above)

2. Definition of the geographic location and extent of the key features. In many cases it may also be appropriate to sub-divide the features and set different conservation objectives for different areas. For example it may be desirable to set different levels of desired forest cover for different valleys and altitudes.

3. Identification of attributes that define the desired condition of the key features, e.g. dominant species composition or species richness for habitats, or breeding population size or breeding success for species. Further examples of attributes that may define the condition of biodiversity features are given below in Box 3.3. For example, an objective might be to maintain broad-leaved forest quality as defined by threshold levels of its attributes of tree species richness, frequency of occurrence of particular keystone tree species, frequency of occurrence of mature trees, shrub density and \% tree regeneration for specific forest types.

This stage also needs to take into account the monitoring resources that are available and the ease of monitoring particular attributes of a feature. For example, establishing the population density of Snow Leopards in the ACA would be extremely time consuming, difficult and costly. It may, therefore, be necessary to set the objectives for this species in relation to an index of relative abundance (e.g. frequency of occurrence) rather than absolute numbers.

4. Establishment of the action to be taken with respect to each feature and its attributes, e.g. increase, maintain, or decrease.

5. Setting of measurable standards for the state or degree of change for each feature's attributes (e.g. maintain $>50 \%$ tree cover)

6. Setting of a time frame for the objective, e.g. increase rhododendron forest cover by $20 \%$ by 2020 . 


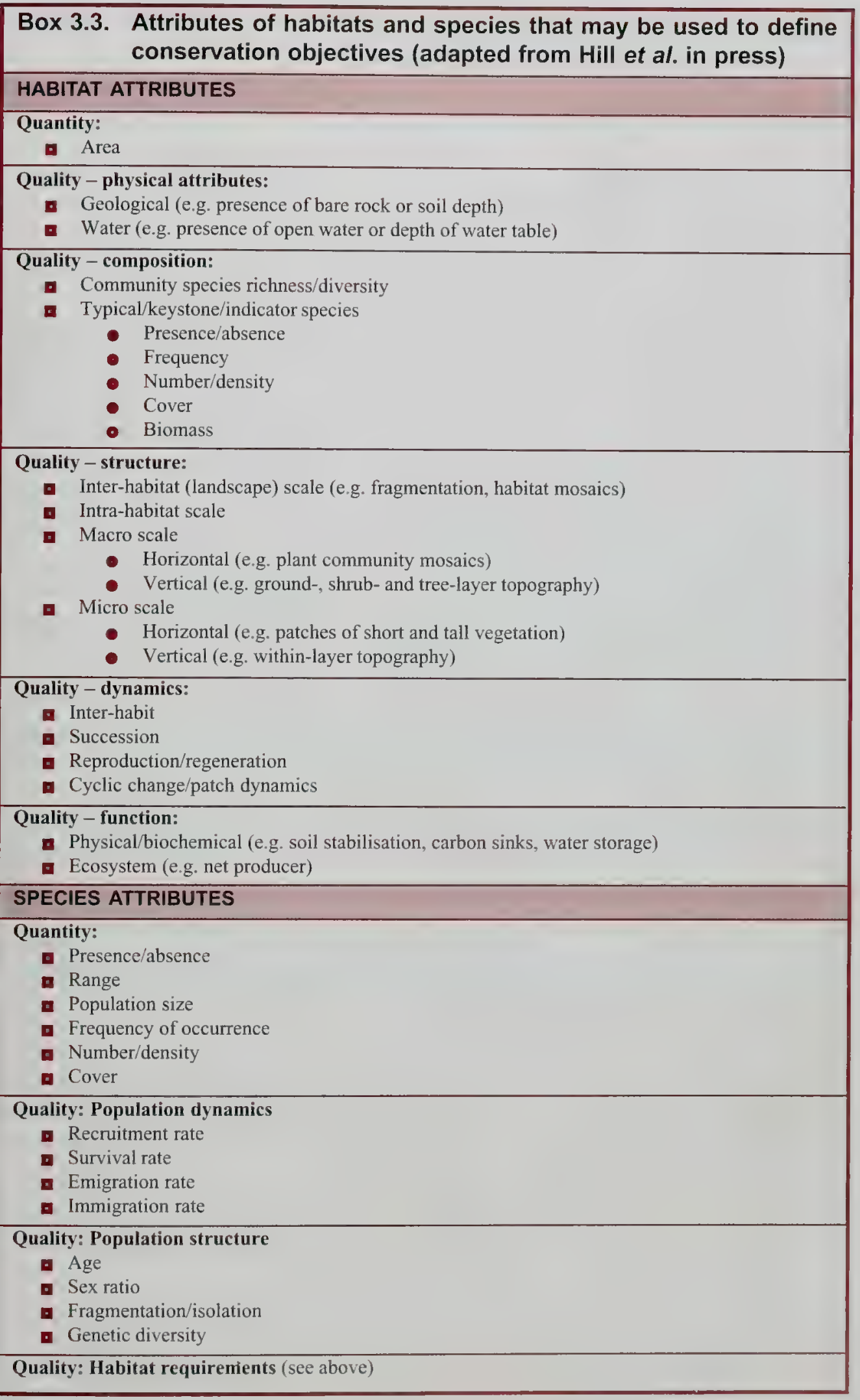




\section{Box 3.4. Biodiversity indicators}

Biodiversity indicators are measures of biological or other features of the environment that reflect to some degree the state of an ecosystem, habitat or other components of biodiversity. Such indicators aim to fulfil three basic functions:

- Simplification, i.e. to provide a simplified measure of a complex feature.

- Quantification, i.e. to enable a numerical measurement to be made of a subjective property, such as habitat quality.

a Communication, i.e. to help understand the condition of a feature.

It is preferable to use a limited number of indicators, so that key conclusions are apparent. The challenge is to strike a balance - the number of indicators should be small to minimise monitoring requirements and to keep the main messages clear, but equally the issues must not be oversimplified.

The presence of a particular species is often used as an indicator, e.g. of habitat quality, or species richness. Such species indicators should preferably be:

a widespread and relatively common in the habitat.

- easy to identify, observe and census.

- well understood with respect to its ecology and interactions with land-uses;

- able to respond rapidly to environmental changes, so that they can provide early warning of detrimental impacts;

- representative of the habitat requirements and ecology of a large number of species;

- of high intrinsic or popular appeal so that they can help motivate action (e.g. 'flagship' species).

The selection process of indicators should include a test to verify that it clearly reflects the changes in the ecosystem for which it was chosen as an indicator.

It is, however, important to note that in many protected areas where resources and capacities are limited and threats to biodiversity are high it will be necessary to set simple objectives that can be monitored easily. Instead of spending scarce resources on quantifying the amount of change it may be sufficient to know if there is a positive or negative trend, why biodiversity is changing, and what are the local perceptions of the causes of change, in order to formulate management decisions.

As an example, some SMART objectives for the state of the Singdi forest (Pasgaon VDC, Lamjung District) key biodiversity feature in the ACA (see Table 3.2) might simply relate to the following four forest attributes: forest area, tree species richness, tree age diversity and the presence of indicator bird species (associated with diverse, native old growth and little impacted forest). Some objectives relating to these attributes might be:

d SF1. Restore the areas of Singdi forest encroached by shifting cultivation to increase forest extent by $25 \%$ in relation to 1995 coverage, by 2015 .

- SF2. Maintain the diversity of native tree species in Singdi forest, such that mean native tree species richness is maintained above 50 species per ha, and non-native tree species account for no more than $5 \%$ of cover in mature forests within the ACA, for the next 50 years. 
- SF3. Maintain the natural forest of Singdi forest by ensuring that less than $10 \%$ of trees are pole class (10-29.9 cm diameter over bark) and more than $30 \%$ of trees are above $30 \mathrm{~cm}$ diameter over bark over the next 20 years.

- SF4. Maintain the wildlife quality of Singdi forest, by ensuring that at least $20 \%$ of characteristic native old-growth forest indicator bird species (as listed in the management plan) are present, over the next 20 years.

As an example of objectives for a species, some SMART objectives for the state of the Snow Leopard population in the ACA might cover the attributes of range, relative population density and breeding success. Some objectives for these attributes might be:

- SL1. Maintain the presence of Snow Leopards in each primary sample block in each of the species' key areas in the ACA.

- SL2. Maintain the relative index of abundance in each of the four key areas in the ACA, over the next 10 years.

- SL3. Ensure successful breeding (rearing of at least one cub) at least once every three years in each key area.

Some other SMART objectives for certain attributes of the state of some biodiversity features in the ACA region (see Table 3.2) might be:

- G1. Maintain the area of utilisable grassland (i.e. that can support over 1 livestock unit per hectare over three months per year) in the Caragana, Lonicera, Carex and Cobressia species grasslands of the trans-Himalayan Rangelands (Mustang and Manang) within $10 \%$ of baseline levels as determined by 2005 satellite image analysis, until at least 2015 .

- CP1. Maintain at least two populations of Cheer Pheasants, with at least 50 calling males in each, in the Southern Annapurna Region over the next 10 years.

- RF1. Increase Rhododendron forest cover in the Ghandruck-Ghorepani region of the ACA by $10 \%$ (according to 2005 baseline surveys) by 2015 .

- IF1. Increase the forest biomass of the intensive forest use zone for fuelwood use in the Ghandruk district by $25 \%$, against 2004 baseline data by 2015 .

\subsubsection{Pressure and response objectives}

Once the overall objectives have been set for the state of each biodiversity feature, then objectives should be set for the pressures that affect it and the responses or actions that may be needed to control the pressures. These would be typically developed as part of the management planning process, with the response objectives guiding the development of a detailed workplan.

Some hypothetical objectives for biodiversity pressures and responses with respect to some biodiversity features in the ACA region are indicated in Table 3.4. 
Table 3.4. Some example pressure and response objectives for some key biodiversity features within the Annapurna Conservation Area

\begin{tabular}{|c|c|c|c|}
\hline $\begin{array}{l}\text { Key Feature } \\
\text { (see above for } \\
\text { objectives) }\end{array}$ & $\begin{array}{l}\text { Pressures on } \\
\text { feature }\end{array}$ & Pressure objectives & $\begin{array}{l}\text { Response objectives (note } \\
\text { some may address more } \\
\text { than one pressure objective) }\end{array}$ \\
\hline \multirow[t]{4}{*}{ Singdi forest } & \multirow[t]{3}{*}{$\begin{array}{l}\text { Fuel wood collec- } \\
\text { tion }\end{array}$} & \multirow[t]{3}{*}{$\begin{array}{l}\text { FW1. Limit fuel wood } \\
\text { collection to less than } \\
3000 \mathrm{~kg} \text { per annum per } \\
\text { household; review in } 2010\end{array}$} & $\begin{array}{l}\text { Carry out forest conservation } \\
\text { awareness workshop in each } \\
\text { village within } 5 \mathrm{~km} \text { of Singdi } \\
\text { forest }\end{array}$ \\
\hline & & & $\begin{array}{l}\text { Build micro-hydro electric station } \\
\text { to supply power to } 200 \text { households } \\
\text { in Singdi village, by } 2010\end{array}$ \\
\hline & & & $\begin{array}{l}\text { Build paraffin fuel store in each } \\
\text { village within } 2 \mathrm{~km} \text { and provide } \\
\text { half price paraffin stoves to } \\
\text { villagers, by } 2008 \text {. }\end{array}$ \\
\hline & Timber collection & $\begin{array}{l}\text { T1. No felling of native } \\
\text { timber within defined } \\
\text { reserve zone until } 2020\end{array}$ & $\begin{array}{l}\text { Increase tree planting in } \\
\text { surrounding intensive use zone } \\
\text { to increase forest cover in } \\
\text { district by } 10 \% \text { by } 2020\end{array}$ \\
\hline \multirow[t]{5}{*}{$\begin{array}{l}\text { Snow } \\
\text { Leopard } \\
\text { Uncia uncia }\end{array}$} & $\begin{array}{l}\text { Disturbance by } \\
\text { trekkers }\end{array}$ & $\begin{array}{l}\text { V1. Maintain less than } \\
0.1 \text { trekkers per } 100 \mathrm{~km}^{2} \\
\text { on average per month in } \\
\text { sensitive parts of key } \\
\text { areas; review in } 2008 \text {. }\end{array}$ & $\begin{array}{l}\text { Identify sensitive areas in each } \\
\text { key area where special permits } \\
\text { are required for trekking / } \\
\text { climbing and limit permits to } \\
<200 \text { per year; review in } 2008 \text {. }\end{array}$ \\
\hline & \multirow{2}{*}{$\begin{array}{l}\text { Decreased prey } \\
\text { numbers (e.g. Blue } \\
\text { Sheep) }\end{array}$} & \multirow[t]{2}{*}{$\begin{array}{l}\text { BS1. Maintain Blue } \\
\text { Sheep densities of }>73 / \\
10 \mathrm{~km} \text { square. }\end{array}$} & $\begin{array}{l}\text { Establish livestock free core zones } \\
\text { in key areas; review in } 2005 \text {. }\end{array}$ \\
\hline & & & $\begin{array}{l}\text { Distribute posters indicating } \\
\text { protection from hunting of Blue } \\
\text { Sheep and other protected } \\
\text { species to all }>2,000 \mathrm{~m} \text { villages } \\
\text { in ACA by } 2006 \text {. }\end{array}$ \\
\hline & $\begin{array}{l}\text { Retaliatory killing } \\
\text { by herders }\end{array}$ & $\begin{array}{l}\text { SLP1. No persecution of } \\
\text { the species in any area. }\end{array}$ & $\begin{array}{l}\text { Set up livestock predation } \\
\text { compensation / insurance } \\
\text { schemes in each village in each } \\
\text { key area by } 2010 \text {. } \\
\text { Preventive measures such as } \\
\text { improved corrals set up in each } \\
\text { village in each key area by } 2010 \text {. } \\
\text { Ensure a Snow Leopard Conser- } \\
\text { vation Committee is established } \\
\text { in each key area by } 2006 \text {, and } \\
\text { in other priority areas by } 2008 \text {. }\end{array}$ \\
\hline & $\begin{array}{l}\text { Hunting for skins } \\
\text { and traditional } \\
\text { medicines }\end{array}$ & $\begin{array}{l}\text { SLP2. No hunting of the } \\
\text { species in any area }\end{array}$ & $\begin{array}{l}\text { Ensure at least two Snow } \\
\text { Leopard conservation awaren- } \\
\text { ess packages organised/provi- } \\
\text { ded in each key area by } 2007 \text {. }\end{array}$ \\
\hline
\end{tabular}




\section{Developing a protected area biodiversity monitoring programme}

\subsection{Introduction}

A protected area biodiversity monitoring programme ${ }^{4}$ requires a great deal of time and effort to develop and implement. It is therefore important to ensure that it is undertaken efficiently, whether or not it involves participatory approaches or field surveys by protected area staff, contractors or others. This requires careful planning, but this is time well spent as monitoring programmes may last many years and poor decisions may not become apparent for a long time; when it is then usually too late to do anything about.

Monitoring programmes should, therefore, be carefully planned to ensure that they are effective (i.e. provide adequate answers to the questions that they were set up to address) and efficient (i.e. collect the required data with as little effort and cost as possible). Many of the most common pitfalls associated with monitoring, as summarised in Table 4.1, can be avoided by careful planning.

\section{Table 4.1. Some common monitoring pitfalls and ways of avoiding them}

\begin{tabular}{|c|c|}
\hline $\begin{array}{c}\text { Common failures in monitoring } \\
\text { programmes }\end{array}$ & Means of avoiding it \\
\hline $\begin{array}{l}\text { Data are collected that are of no use, and/or key } \\
\text { questions cannot be answered. }\end{array}$ & $\begin{array}{l}\text { Focus on determining if conservation objectives } \\
\text { are being achieved, and identify data requirements } \\
\text { and analytical methods carefully. }\end{array}$ \\
\hline Poor design leads to inconclusive results. & $\begin{array}{l}\text { Plan carefully and test with pilot surveys and } \\
\text { analysis. }\end{array}$ \\
\hline $\begin{array}{l}\text { Multiple observers differ in field skills and use } \\
\text { inconsistent methods. }\end{array}$ & $\begin{array}{l}\text { Document precisely the methods to be used and } \\
\text { ensure observers have been trained in using the } \\
\text { methods, have practiced them and follow them. }\end{array}$ \\
\hline $\begin{array}{l}\text { Methods are changed during the monitoring } \\
\text { programme, and thus survey results cannot be } \\
\text { compared. }\end{array}$ & $\begin{array}{l}\text { Plan and test the methods and then stick to them. } \\
\text { If changes are absolutely essential document them } \\
\text { and if possible calibrate effects of changes. }\end{array}$ \\
\hline $\begin{array}{l}\text { Inappropriate methods are chosen for habitats or } \\
\text { species. }\end{array}$ & $\begin{array}{l}\text { Know your habitat and species, and if necessary } \\
\text { carry out pilot field tests. } \\
\text { (Cont.) }\end{array}$ \\
\hline
\end{tabular}

${ }^{4}$ A protected area biodiversity monitoring programme is the whole series of related sunveys and analysis that aims to establish whether or not all the specific biodiversity objectives for the area are being achieved. 


\begin{abstract}
A few large samples are taken rather than many small ones, so natural variation cannot be accurately measured.
\end{abstract}

Natural fluctuations in populations and habitat conditions obscure management changes.
Use many small samples in preference to a few larger ones, and if possible carry out pilot surveys to estimate variation and calculate the required number of samples to detect significant changes.

Ensure that sampling is sufficient in terms of numbers and frequency and carried out using appropriate methods

Locations of permanent sample sites are not properly marked and recorded so that the same areas are not revisited.

Carefully mark sample locations with more than one marker, designed to last a sufficient length of time. Photograph the site, take GPS coordinates and map the location.

Biased results are obtained because samples are purposefully taken in good/interesting areas of habitat or favoured sites for species etc.

Spurious results may be obtained by comparing data using the same methods in different habitats because the results are incorrectly assumed to be directly comparable.

Results are assumed to be completely accurate and biases are ignored (e.g. birds counted are assumed to be all the birds present).

The data cannot be analysed statistically.

Data are lost, either physically or within organisations.

Take random or systematic samples (see Section 4.9).

The efficiency of methods may vary between habitats, so take care with inter-habitat comparisons or measure and calibrate habitat effects.

Identify and document biases, and standardise methods to maintain consistent biases.

Ensure that planning includes identification of the statistical methods to be used and their data requirements.

Ensure data are copied as soon as possible, maintain records of datasets and their locations. with full documentation of methods used to collect them. Inform others of the data.

Data are not analysed because the biologists lack the skills to do so.

Ensure that the methods of analysis are identified at the planning stage and that suitably qualified staff are available and responsible for analysis of the results.

Managers, other staff and external stakeholders do not use monitoring results because the data are not useful or not trusted.

Monitoring programmes are not sustained and results remain unused due to loss of institutional support.
Spread ownership of the programme by involving managers and other stakeholders in planning of monitoring.

Ensure that there are adequate long-term resources for the whole programme, and use and increase the local capacity for participatory monitoring. Share results with a broad community to increase possible sources of funding.

Good planning of a monitoring programme involves consideration of a number of key issues, which are outlined in the diagram in Figure 4.1. Although the diagram suggests that the planning is a step by step process, in practice it requires iterative decision making, because decisions may have to be revised according to the outcome of later decisions. In other words, some decisions may have to be reconsidered at later stages of the planning process. For example, it may be necessary to reconsider the frequency of monitoring of some features, once the overall programme has been costed. 
Figure 4.1. Important steps in planning a biodiversity monitoring programme

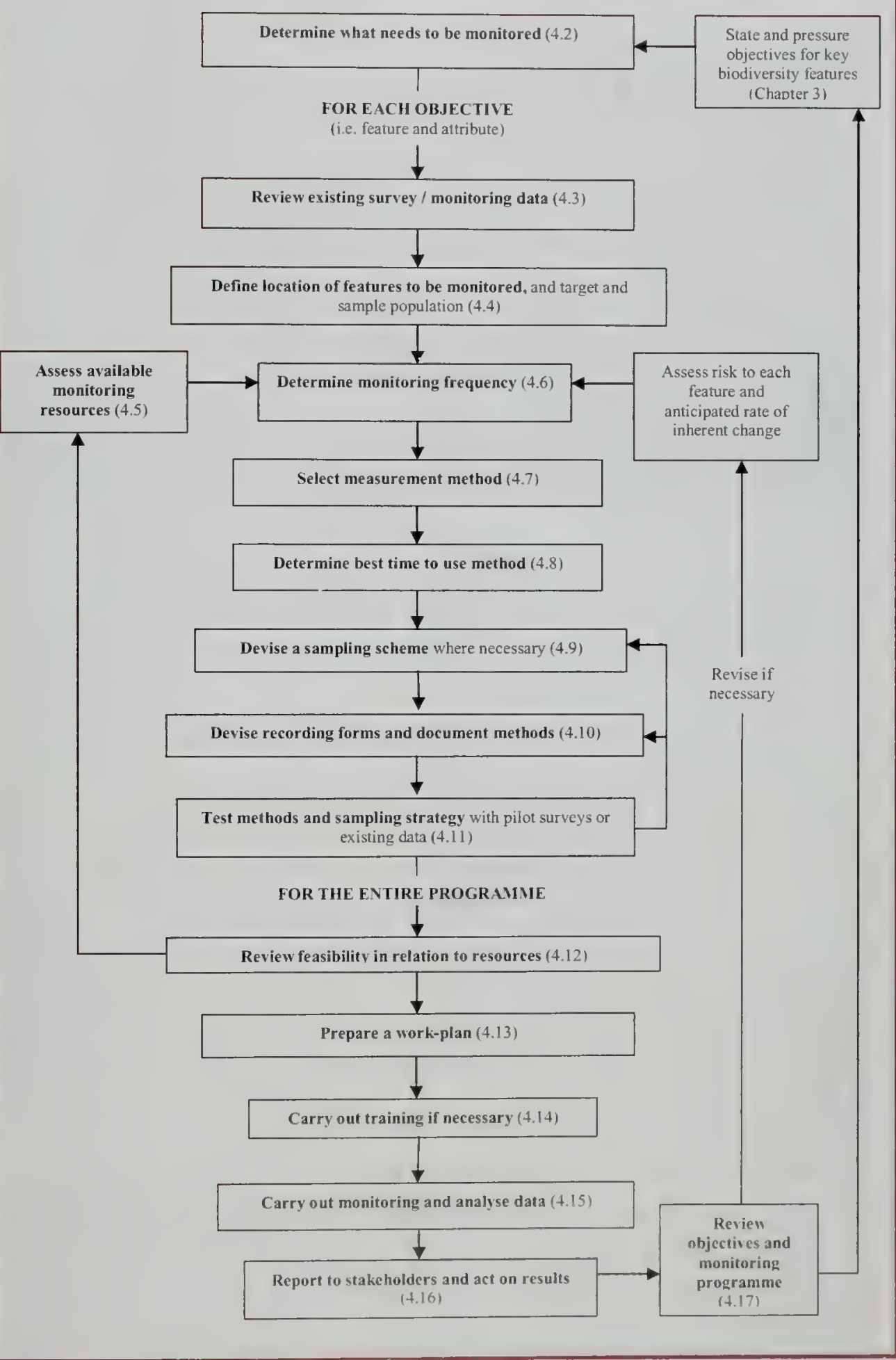

Note: Figures in brackets refer to relevant text sections 


\section{Plate 4.1 Cheer Pheasant observation}

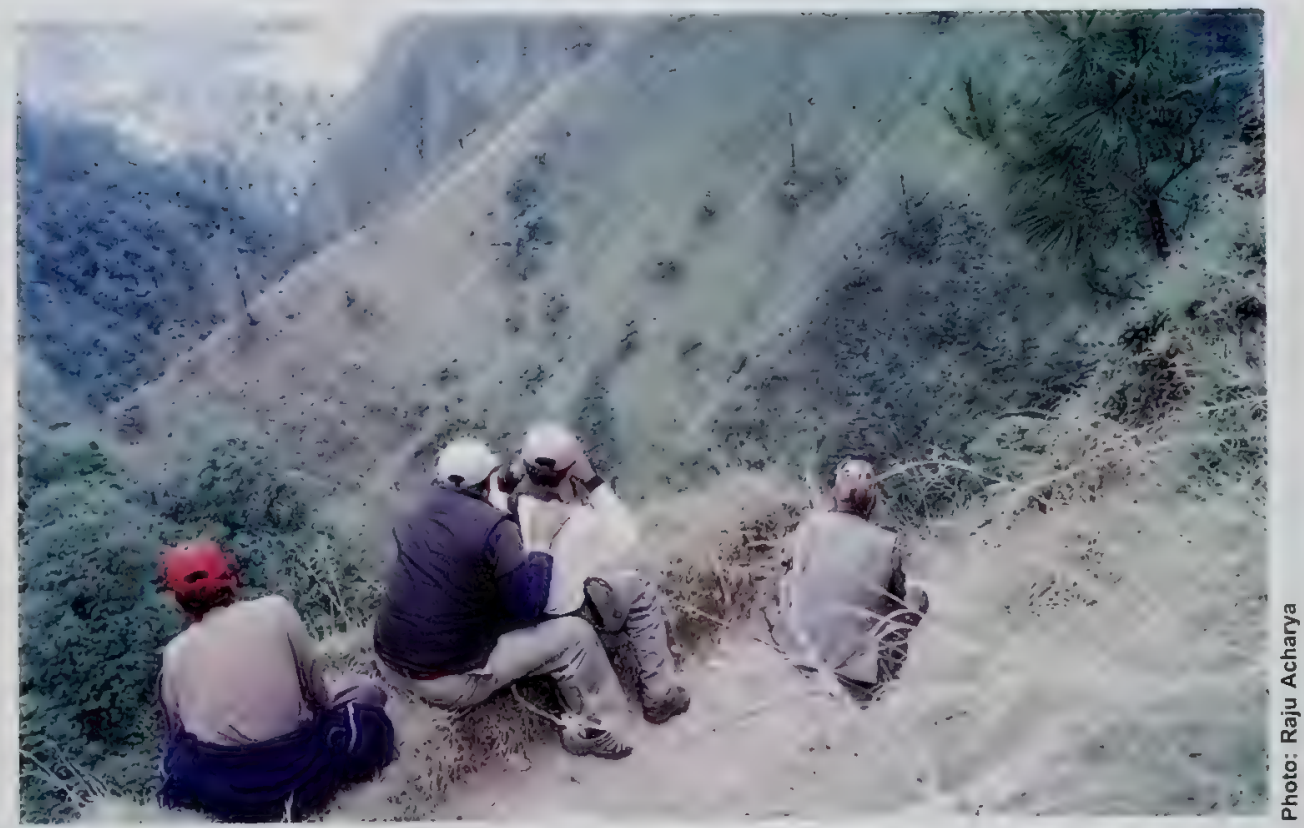

Each step outlined in Figure 4.1 requires consideration of a number of key decisions (which are further described in Sections $4.2-4.17$ below. These decisions will require careful consideration of your monitoring aims, available information on the biodiversity features to be monitored, the physical nature of the areas to be monitored (e.g. size, topography, climate, accessibility) and available resources (time, funding, expertise).

Some recommended sources of further information on biodiversity monitoring are listed in Box 4.1.

\section{Box 4.1. Recommended references for further information on biodiversity monitoring strategies and methods}

See reference list for full citation details.

Doak and Pollock. Statistical / monitoring tools for the design and analysis of conservation monitoring data http:/www.biology.ucsc.edu/people/doaklab/natconserv/index.html

Elzinga et al. (2001). Monitoring plant and animal populations.

Fancy. Monitoring Natural Resources in our National Parks. http:/www.nature.nps.gov/im/ monitor/

Feinsinger (2001). Designing field studies for biodiversity conservation.

Goldsmith (1991). Monitoring for conservation and ecology.

Hill et al. (in press). A species and habitat survey, evaluation and monitoring handbook.

Krebs (1999). Ecological methodology 2nd ed.

Southwood (1978). Ecological methods.

Spellerberg (1991). Monitoring ecological change.

Sutherland (1996). Ecological census techniques. 


\subsection{Determine what needs to be monitored}

\subsubsection{Monitor objectives for the key biodiversity features}

Clearly and explicitly defining the purpose of the monitoring programme is probably the most important step. Failure to do so may result in data being collected that are of little value, or of important data requirements being overlooked.

In these guidelines for monitoring biodiversity in protected areas, we recommend that monitoring should focus on establishing whether or not the conservation objectives for the area are being achieved (see Section 3.6). Thus the first stage in developing a monitoring programme is to ensure that you have clearly defined conservation objectives. Key features of biodiversity importance in the protected area should be identified and these should be the focus of management actions and monitoring. Each key feature should have clear (SMART) conservation objectives set for it. Ideally these will have been already established during the production of a management plan or similar document (as described in Section 2.1).

\subsubsection{Monitor states, pressures and responses}

We have also recommended that SMART objectives should be set for regulating pressures on key biodiversity features, and for management responses to pressures which result in changes in the state of features (in accordance with the Pressure - State - Response framework described in Section 3.5). Thus in addition to establishing if conservation objectives are being met (i.e. establishing the state of the key feature) monitoring should also establish if objectives relating to pressures and responses are being achieved. For example, a monitoring programme for Snow Leopard might include:

\section{State monitoring}

- Presence of Snow Leopard throughout the ACA (from reported sightings).

- Relative abundance of Snow Leopard in selected key areas within the ACA.

- Successful breeding in key areas (i.e. presence of juvenile animals in the population).

\section{Pressure Monitoring}

- Livestock abundance and seasonality in grasslands in Snow Leopard range within the ACA.

- Abundance of Blue Sheep and other natural prey species in Snow Leopard range within the ACA.

- Condition of high altitude grassland / shrublands in key areas for Snow Leopard.

- Numbers of herders within key areas for Snow Leopard.

- Disturbance by trekkers / climbers etc in key areas for Snow Leopard.

- Cases of killing of Snow Leopards (persecution / retaliatory killing etc).

\section{Response monitoring (illustrative actions)}

- Number of anti-poaching patrols carried out in high risk areas

- Proportion of communities with established Snow Leopard Conservation Subcommittees

- Number of meetings held with village communities, to raise awareness of threats and to advise on livestock protection measures that avoid the need to kill Snow Leopards 
- Success in rangeland restoration measures (livestock exclusion)

- Extent of wildlife conservation awareness camps and inclusion of primarily herders and farmers who are intrinsically dependent on forest and grazing land (awareness creation)

- Number of legal actions initiated by the KMTNC-ACAP as per the rules and regulations (law enforcement aspect)

- Inclusion of wildlife conservation as a subject in the school $\mathrm{CE}$ curriculum

When considering pressure and response monitoring requirements it may be found that some monitoring activities can be combined with those needed for other key features. For example, grassland/shrublands is a key biodiversity feature in its own right, and therefore monitoring of the state of grasslands meets the requirement to monitor pressures on Snow Leopards (through impacts on Blue Sheep and other important prey).

These guidelines focus on biodiversity monitoring, and therefore the monitoring of abiotic pressures and management actions (i.e. responses) is not within its scope. The rest of this chapter provides detailed guidance on how to plan and undertake monitoring of objectives that relate to biodiversity components.

\subsection{Review existing data}

It is important to establish whether monitoring has previously been undertaken, including the features and attributes covered, the methods used. the time-scale and frequency over which it took place, and whether or not it is ongoing. This may require careful investigation as it is not unusual for the results of monitoring studies to be forgotten, especially where they are unpublished and collected by an external organisation, or where personnel have since moved on.

Data from previous monitoring programmes or ad hoc surveys should be used in the assessment process (described in Chapter 3 ) to identify key features to be monitored, if these have not been identified in a management plan, and to help assess the appropriateness of potential survey methods and sampling strategies.

Where possible and appropriate, monitoring should build on existing data collection programmes (e.g. government forest inventories), institutional arrangements (e.g. ACAP regional structure and CMACs) and local community activities (e.g. forest patrols, sheparding). But previous monitoring programmes should not be simply repeated without careful consideration of their suitability, as they may have been established to meet different objectives. However, where existing programmes are likely to contribute to current objectives they should be continued and developed if necessary. Where appropriate, existing methodologies should be followed to maintain the validity of long-term datasets. It may also be useful to use existing fixed marker systems or permanent quadrats.

If prior surveys have not been carried out and the identity and condition of biodiversity features of importance is in doubt, then it may be necessary to carry out a baseline survey before a detailed monitoring programme can be planned. It is necessary to establish the baseline condition of features so that appropriate conservation objectives for them can be set and any subsequent changes in them detected. 


\subsection{Define the location and boundaries of the biodiversity features to be monitored}

It is vital to clearly define the geographical area or species population that you wish to monitor and draw conclusions on. This requires consideration and definition of four types of population that you may be interested in: biological population, target population, sampled population and statistical population (Elzinga et al. 2001).

A biological population is "a group of organisms of the same species, present in one place at one time". For example. all the plants of a particular wetland species that occur at an isolated wetland might be appropriately defined as a biological population. But in many cases biological populations are difficult to define in practice because the "place" occupied by a species is often not clearly definable. This is particularly the case for mobile species or species that have a wide capacity for dispersal, so that there is much emigration and immigration between apparently geographically separate populations.

Where feasible it is best to monitor on a biological population basis. This means that different biological populations are monitored (or analysed) separately and whole populations are monitored where possible. In practice this is often difficult to achieve, especially for large or mobile populations, as protected areas often cover only a small part of the population of such species. However, within the ACA it might be possible to conclude that the main west-east Annapurna mountain chain divides the area into two broad areas which may support distinct and separate populations of many species. Monitoring may therefore need to consider the population status of each of these separately.

In many species biological populations will extend beyond the ACA boundary. Where such extensions are small then monitoring should cover the entire biological population rather than be constrained by the administrative boundary of the ACA. This enables more ecologically meaningful conclusions to be drawn from the monitoring. For example, one might imagine a situation where a bear population is found within a valley, half of which occurs within the ACA. Monitoring of the ACA section only might reveal that bear numbers have declined. But monitoring of the whole valley might indicate they have merely moved to another section of the valley outside the ACA. Although the loss of bears from the ACA may be undesirable for some reasons, the conclusion that the bears have moved is much less serious than the erroneous conclusion that they have declined.

In practise monitoring normally focuses on part of the biological population, typically that which is within the protected area. Or sometimes we may only be interested in monitoring part of the population within the protected area, if for example, part of the area is being managed specifically for a habitat or species, although they may occur elsewhere in the protected area. The population that we are interested in can be called the target population (see Figure 4.2a) and the area in which it is found is the target area.

It is sometimes possible to monitor a whole feature across a target area. For example it may be possible to reliably measure the full extent of a habitat feature by aerial photography or carry out a complete census of a localised and conspicuous species. But more often it will be necessary or more efficient to monitor a feature by assessing samples. The area over which samples may be drawn then defines the sample area and sample population. 
The statistical population is the entire set of observations across all the samples, from which statistical inferences are made.

Ideally the sample population should be the same as the target population, but in many cases the sample population will be different due to practical constraints. When this occurs it is vital to recognise the difference between your target population and your sampled population so you know the limitations of your data. It is only possible to draw valid statistical conclusions about your sampled population.

When the target population has irregular boundaries, it may be most practical to redefine a new sample population by fitting a regular shaped polygon over the bulk of the target area. This newly defined area, referred to as a macroplot, becomes the sample population. Macroplots are relatively large areas, with sampling units such as quadrats, lines or points (see Section 4.7) located within them. They facilitate the positioning of sampling units. Macroplots are usually permanently defined to ensure that the same area is sampled on each sampling occasion.

Sample populations may also differ from target populations because areas cannot be sampled for practical reasons, because for example they are too steep or wet or inaccessible for other reasons (see Figure 4.2).

If a target population covers a large area then it may not be feasible to sample the whole area because of time and resource constraints. Sampling over a large area will usually result in widely scattered sample locations, which will entail considerable amounts of travel time. This is particularly a problem in areas such as the ACA where there is no means of transport, paths are limited and much of the terrain is extremely steep and hazardous. It is therefore necessary in such circumstances to restrict the sampling area to a smaller subset of the target population. This can be accomplished by placing a random sample of macroplots (primary plots) within the target population. Further sampling (i.e. with secondary plots) is then carried out within the primary plots (leading to a two-stage sampling design as described further in Section 5.5). Because the macroplots are randomly selected from within the entire target area, the sampled population and the target population are the same, and therefore statistical conclusions apply to the entire target population.

However, if the target population is very large and difficult to sample, then it may be necessary to restrict sampling to a few selected key areas. For example, it is not practical to attempt sampling of Snow Leopard occurrence over its entire target population (i.e. the entire extent of suitable habitat for the species within the ACA). Although the species is probably widespread, in many parts of its range within the ACA it may only occur irregularly and at very low densities. It is therefore only practical to identity and monitor (by field surveys) a few key areas where Snow Leopards are known to occur. Samples are then taken within each key area. This is again two-stage sampling, but in this case the sampled population is only each key area. Statistical inferences may only be validly drawn for each key area. Values from the key areas should never be averaged because they are not randomly drawn samples; key areas are selected (normally subjectively) with a particular intent (e.g. to study areas of known importance for a species). 
Because statistical inferences can only be made to the key areas that are actually sampled, it is important to set conservation objectives that are specific to each key area, rather than the target area as a whole (unless other means exist for monitoring the whole target area). Where necessary it is equally important to clarify within management plans that actions may be taken according to the results of the monitoring in key areas, despite the fact that they are not necessarily representative of the situation across the whole target population.

\section{Figure 4.2. Example illustrations of target populations, sample populations and the use of key areas}

Key:

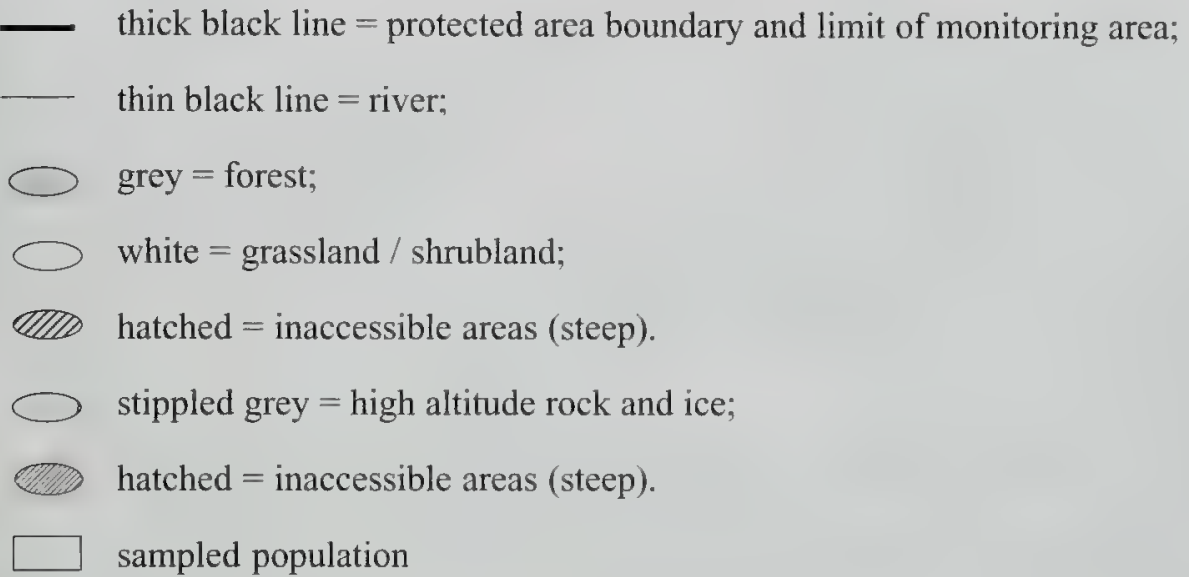

4.a. Forest bird monitoring. The target population is all the solid grey (forest). The sampled population is the grey area excluding the hatched grey areas (inaccessible land). Survey plots are randomly distributed (see Section 4.9 ) over the sampled population (white squares).

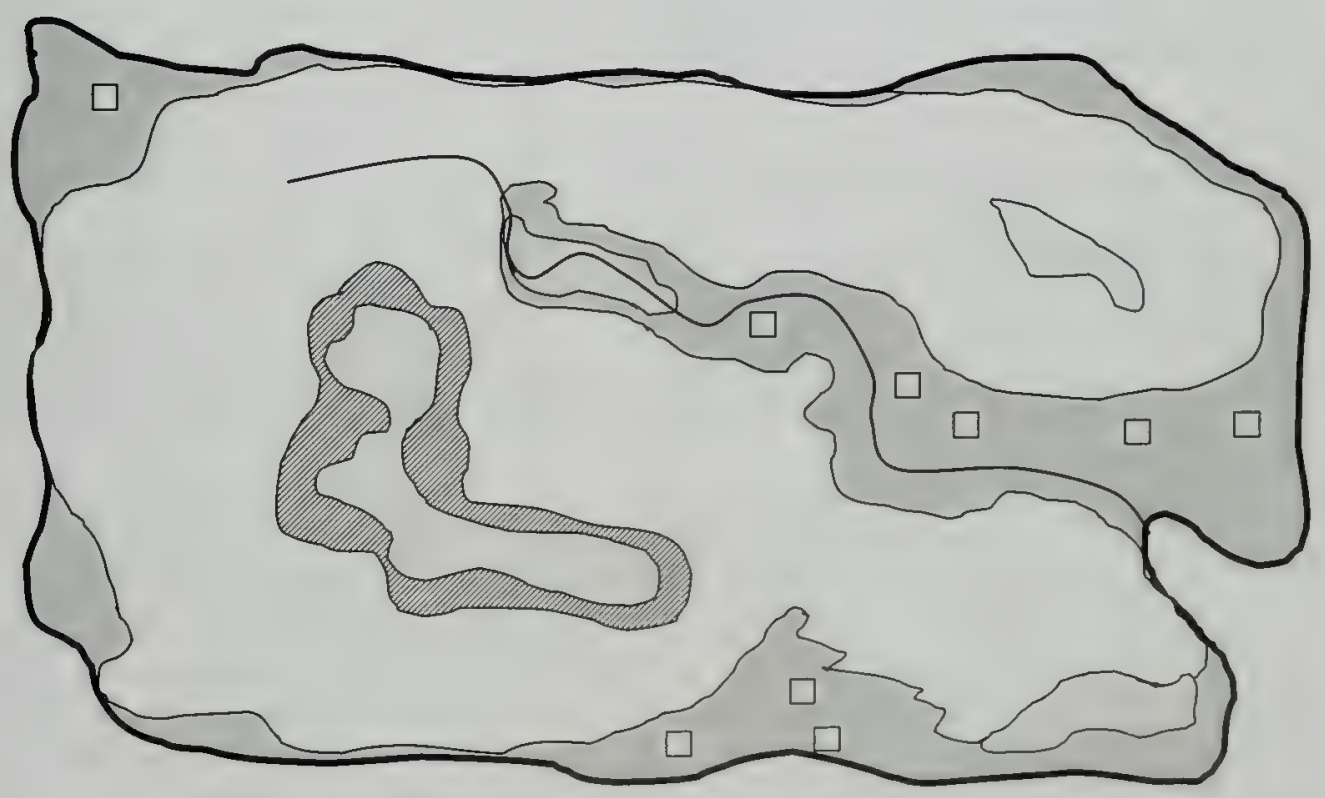


4.b. Snow Leopard monitoring in key areas. The target population is the white area (i.e. suitable grassland / shrubland habitat). The three key areas (dotted lines) excluding the hatched black and white areas (inaccessible land) are three separate sampled populations. Survey plots are distributed by restricted random sampling (see Section 4.9) in each key area.

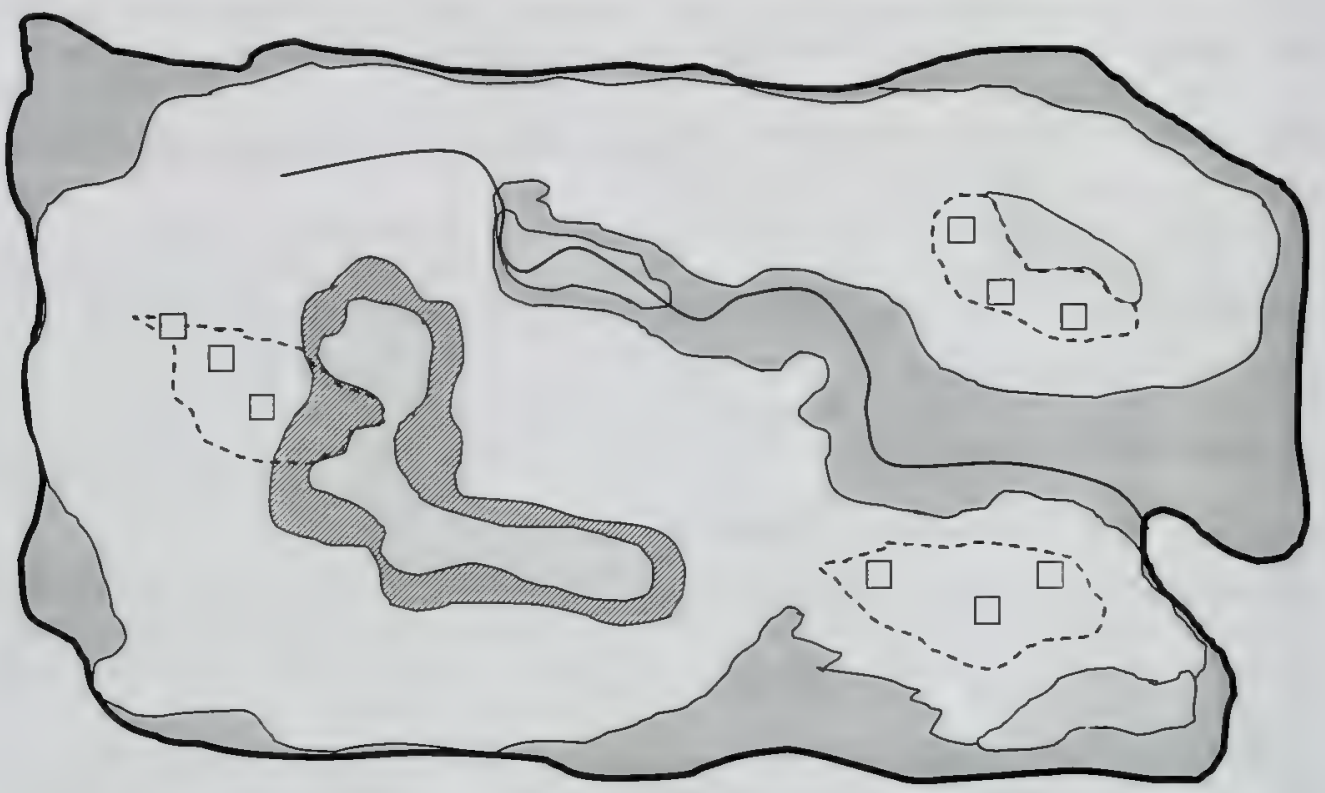

\subsection{Assess available monitoring resources and prepare budget}

It is particularly important to take into account available resources (e.g. staff, time, expertise, transport, funding) when planning a monitoring programme. Many scientific monitoring activities are time-consuming, expensive and require experienced personnel. Careful consideration therefore needs to be given to what is achievable when setting monitoring objectives, especially in the long-term. Monitoring will be of no value if it cannot be repeated. Some of these problems can be alleviated by the careful targeting and efficient design of monitoring programmes, as suggested in Box 4.2 .

\section{Box 4.2. Approaches to minimising costs of monitoring in protected areas}

The key aim of monitoring in protected areas is to establish whether or not conservation objectives are being met. Even where monitoring resources are severely limited, this may be achieved by taking the following actions:

1. Prioritise monitoring for features that are of highest conservation interest and at highest risk (based on a risk analysis and estimate of the probability of each possible impact and its likely magnitude).

2. Set simple conservation objectives where possible and restrict monitoring to what is required to test if these objectives are being met (e.g. if the aim is to maintain the presence of a species do not use more time consuming methods that estimate densities).

3. Monitor pressures (see Section 3.5) more frequently than the state of features, as these are often easier to measure and can provide early warning of potential problems. But do not restrict monitoring to pressures. Carry out occasional state monitoring, and use pressure monitoring to trigger further state monitoring if pressures increase.

(cont.) 


\section{Box 4.2. Approaches to minimising costs of monitoring in protected areas (cont.)}

4. Focus on key areas (see Section 4.4) where it is not possible to cover all areas effectively, but realise that this restricts one's ability to draw conclusions about the protected area as a whole.

5. Only monitor as frequently as necessary (see Section 4.6, i.e. in accordance with expected rates of change or risks). For example, there is little to be gained from monitoring forest condition at less than 5 year intervals (unless pressure monitoring or casual visits indicate a sudden event e.g. fire).

6. Use the most cost-effective methods, but be aware that these need not necessarily be the most simple. For example, remote-sensing may be the most cost-effective method for monitoring habitat extent Consiujer participatory approaches where appropriate, but ensure that they really are cost-effective (as they may take a lot of time to set up and run over the long-term) and provide the data that you need consistently and reliably.

7. Be clever and use efficient sampling strategies. For example consider multi-stage sampling to cut down on travel time between sites (see Section 4.9). And use permanent sample sites where feasible. Although these may be time-consuming to establish they are much more efficient in detecting changes in the long run provided that a sufficient number are established and located randomly.

8. Use a phased approach to develop monitoring when major project funding is available

Participatory monitoring methods may also be more sustainable and provide other advantages, such as increased ownership of results (e.g. see Danielsen et al. 2000, Danielsen et al. 2003). However, although participatory biodiversity monitoring can be cheaper than more conventional scientific monitoring, this is not always so. Focussing monitoring on pressures, or carrying out threat reduction assessments (Salafsky and Margoluis 1999) can also reduce costs and training requirements etc, but the monitoring of pressures should never replace monitoring the state of key features. Pressure monitoring may, however, be carried out, more frequently than state monitoring, and then used as a possible trigger for more intensive and frequent state monitoring if pressures appear to increase and thus warn of possible impacts (see Section 4.6).

A preliminary budget should be defined at this stage of the process since there is no point developing programmes without the funds to implement it. The items of the budget should at least include costs of external data (e.g. aerial photographs), staff time, equipment, local meetings, transportation, stationery, and other operational costs. Funds for publicity and dissemination are important as well. Staff and local participants may need to be trained in field methods, record keeping and data analysis, depending on which methods are to be used.

The budget may need to include payment to participating stakeholders, particularly villagers. For local communities, especially in the case of poor farmers, being involved in biodiversity monitoring is extra work which takes time and money. It is fair to offer a fee to take account of these costs borne by local people, keeping in mind that this will be temporary. After developing the rest of the methodology, i.e. after determining how to monitor each objective and how to document and disseminate the results, the budget should be reviewed and finalised. If a relatively large amount of short-term funding is available. e.g. as a threeyear development project, then this can be used to develop monitoring through a phased approach: 
- Use the project funding to do baseline assessments, test monitoring methods and carry out training to develop capacity.

- Validate and calibrate simple methods against more detailed methods during the project phase.

- In the longer-term, adapt monitoring according to increased knowledge, and rates of environmental change and risk (e.g. use simple methods if these have been shown to provide reliable results, and reduce quantitative monitoring of features that are of low risk of sudden change).

\subsection{Determine the monitoring frequency}

The frequency at which monitoring is carried out is a key factor affecting the cost of monitoring. One should therefore avoid monitoring more frequently than is necessary. The likely rate of change in each feature as a result of natural events and management interventions is of key importance in deciding how often monitoring visits should be carried out. Thus, for example, it may be appropriate to visit forests at five-year intervals because major changes in such habitats are normally very slow in the absence of disturbance. But bird populations may vary considerably from year to year, so surveys may need to be carried out annually if resources allow.

But unexpected events may affect the biodiversity features. Monitoring programmes should therefore incorporate sufficient flexibility to deal with unforeseen, potentially rapid and catastrophic events (e.g. storms and fires). Additional very basic inspections may be needed to detect such events and then additional monitoring can be designed to establish the condition of a site.

A general procedure for determining the appropriate frequency of monitoring for a particular feature is:

1 Select an interval consistent with:

a the likely rate of change of the feature in relation to intrinsic natural rates of change and known management influences, taking into account the precision with which that change can be measured;

- any reporting requirements (e.g. to project donors); and

- the availability of funds for monitoring.

2 Aim to make a detailed assessment of the attribute at the required interval (e.g. for a protected forest area, aerial photography may be required at intervals of 10 years).

3 Assess the risk of change from external factors.

Unless very frequent monitoring is required, aim to make a basic inspection of the features and their attributes more frequently for signs of abrupt change (e.g. for forests, a basic inspection at intervals of 3 years may be appropriate). 


\subsection{Select measurement methods}

Choosing the most appropriate method for measuring each key feature and its attributes, or pressures on it, is another critical step in planning a monitoring programme. Essentially one should aim to use the most cost-effective method that provides an adequate assessment of whether or not the conservation objective for the feature (i.e. its state) or the management objective (i.e. relating to pressures on it) are being met. Very often the most cost-effective method may be the simplest, but this is not always the case. Well planned and implemented scientific studies may in the long-term provide better value for money than very simple subjective methods that may produce results of little value.

The choice of methods needs to take into account some key questions, as outlined in Figure 4.3 and described below (much of which is based on Hill et al. in press). From this brief discussion of potential measurement methods it is clear that there are a number of important issues to be considered. However, it is difficult to make the best choices without practical experience and data, and therefore as discussed in Section 4.11 it is highly recommended that methods are tested in some preliminary field trials before a final selection is made.

\section{Plate 4.2 Snow Leopard habitat in ACA, Nepal}

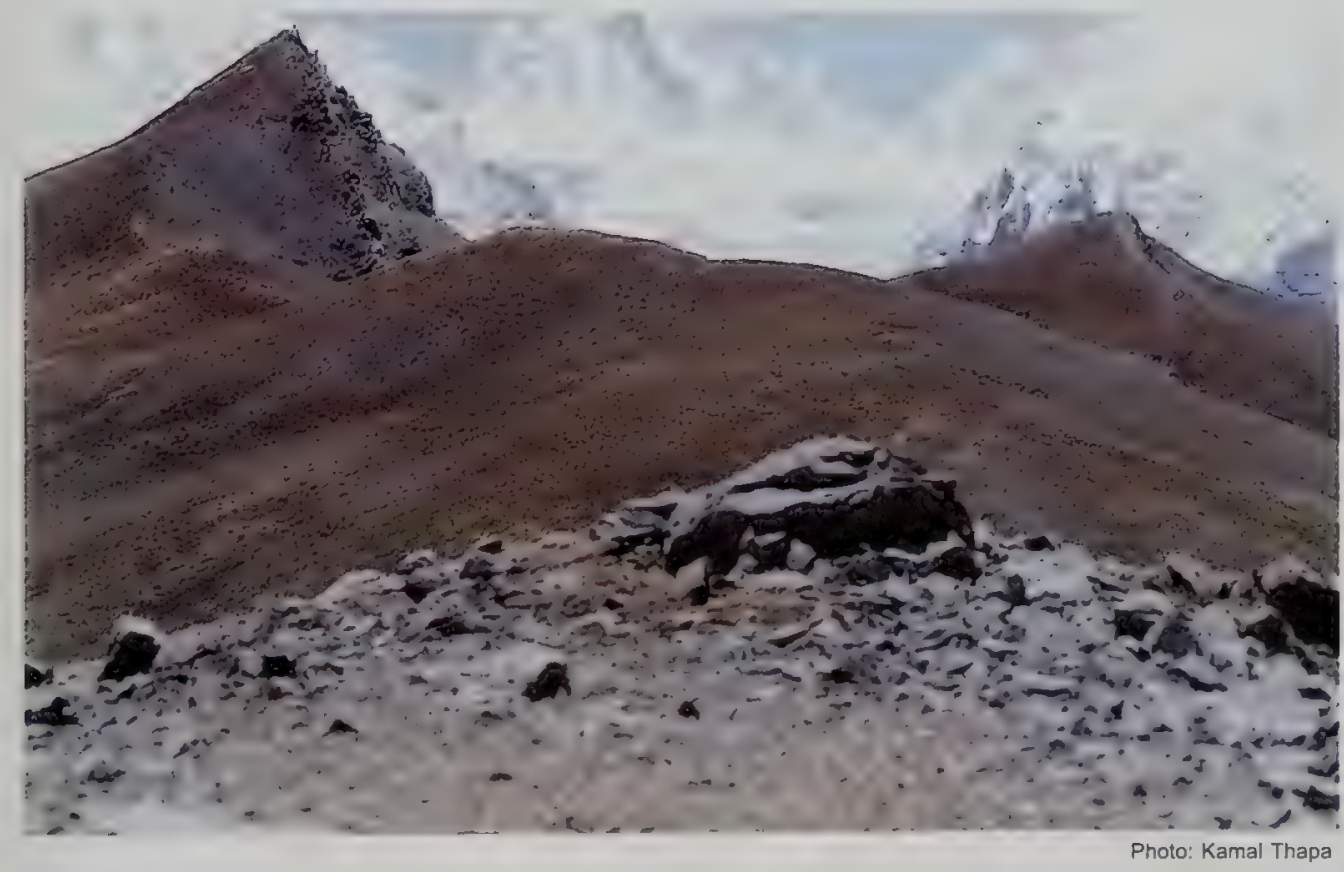


Figure 4.3. Selection of methods for measuring attributes of each feature

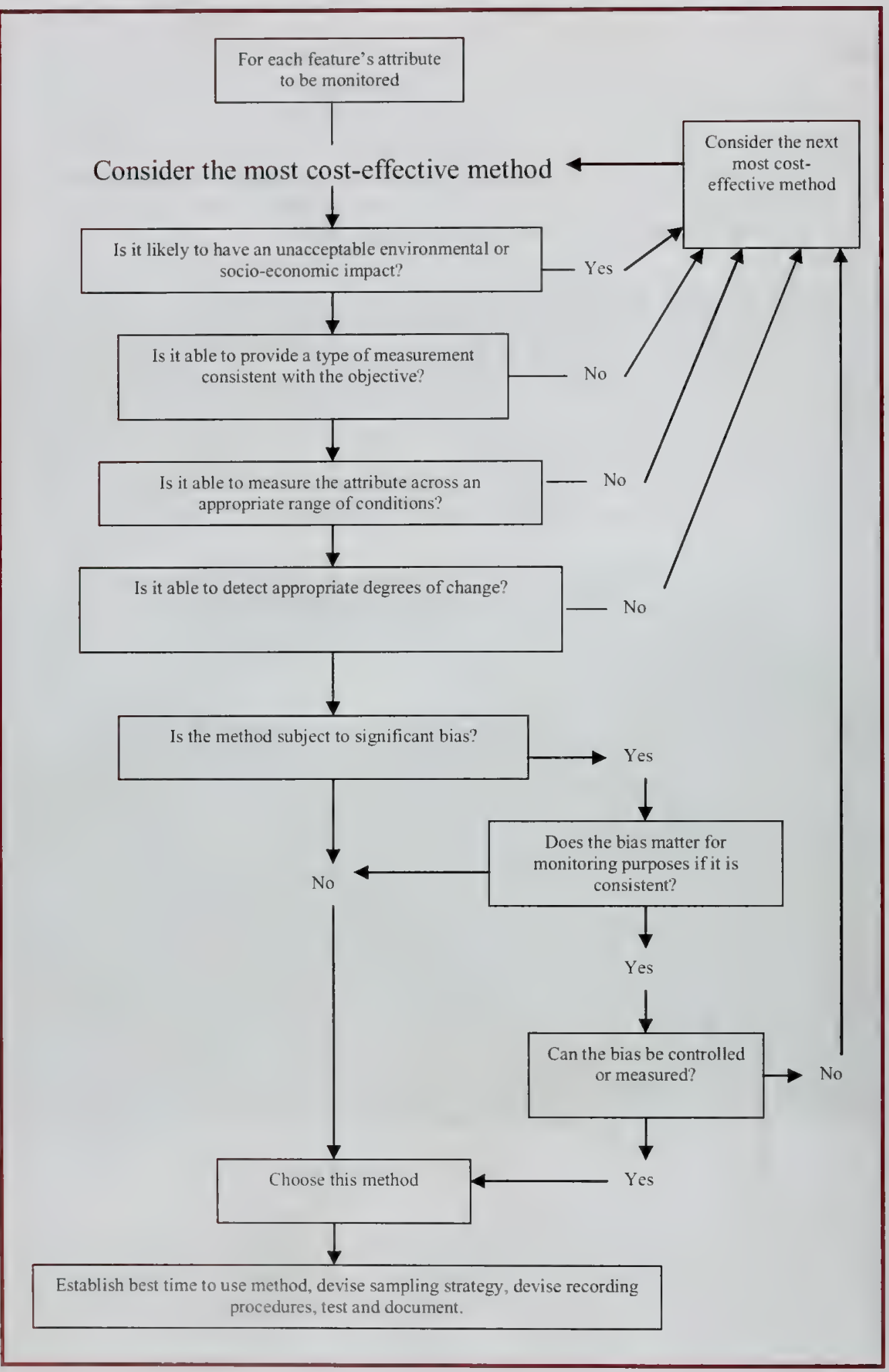




\subsubsection{Ensure that the method will not have unacceptable environmental or socio-economic impacts}

Unfortunately, there are many documented cases where research and monitoring programmes have merely measured and recorded the damage caused by their own activities. Therefore, great care should be taken to ensure that the methods chosen will not cause any damage, and the following precautions should be observed:

- Do not use destructive sampling methods unless absolutely necessary.

- Ensure that important wildlife, natural resources, livestock and crops are not damaged during field surveys (e.g. by trampling).

- Minimise disturbance to sensitive species.

- Do not use vehicles on sensitive habitats unless impacts can be avoided.

- Position fixed sampling locations sensitively and avoid or minimise damage during their establishment.

- Ensure any fixed or temporary structures that are left unattended do not pose a risk to wildlife, livestock or local people.

- Avoid excessive re-visiting of sites and sampling locations.

\subsubsection{Choose the most cost-effective method that provides the appropriate type of measurement}

One of the key questions when planning monitoring is whether to use subjective assessments (e.g. villagers are asked if fuel wood collection has increased, decreased or remained the same over the last year), or objective measurements (such as by counting the amount of fuel wood logs in a sample of local houses over the year in question). Subjective assessments are generally easier and, therefore, more readily applicable to participatory approaches. As a result they may be cheap and a lot of data (or samples) can then be collected per unit cost. However, many studies have shown that people vary greatly in their subjective assessnents. Subjective assessments are also usually more biased than objective measurements, and very importantly, such biases may vary between occasions. One must also be aware that such biases may result from vested interests or subconscious wishes. There are many cases where local resource users (e.g. fishermen) have claimed that resources are not being overexploited, despite overwhelming scientific evidence to the contrary.

One must therefore carefully weigh up the risks of relying on subjective assessments, and they should not be used by themselves when impacts on an important feature could be rapid, substantial and irreversible. Subjective assessments may, however, be particularly useful if validated, and even better, calibrated against objective methods. They may also be useful as a simple early warning system of impacts on features that are in good condition and at low risk of rapid change.

It should also be remembered that the use of objective methods does not preclude participation by local communities. The scientific principles described in these guidelines can be applied to participatory approaches, and we should not underestimate the abilities of local people to record detailed and complex data, though the analysis and generation of useful results may require external support. On the other hand, highly technical and labour intensive scientific methods are more often than not unsustainable in the long-term. 
Another key consideration is whether to use quantitative, semi-quantitative or qualitative methods.

- Qualitative data: e.g. single assessments of presence / absence of a feature in a site or area with no indication of abundance. These data can be combined to create frequency datasets, which are semi-quantitative data.

- Semi-quantitative data: e.g. data that can be analysed mathematically, but do not provide absolute measurements of the attribute. Relative abundance is an example of this type of data. For example, with relative abundance data one may say that species $\mathrm{A}$ is twice as abundant now compared to 10 years ago, but one cannot know how abundant the species is or was.

- Quantitative data: e.g. direct counts, estimates or indices of absolute abundance, area etc.

In practice it is often necessary to use simple qualitative methods in protected area management monitoring, where resources and capacities are limited and threats to biodiversity are high. However, this is not necessarily a significant problem as qualitative data can normally be obtained more quickly and cheaply than semi-quantitative data, and especially quantitative data. The ability to take more samples than quantitative methods may then overcome the greater variation in measurements resulting in greater precision overall. In other words it may be better to take a lot of simple measurements than a few reliable estimates. For example, with the same amount of limited time and resources it may be possible to assess the population status of Snow Leopards in the ACA by measuring its presence or absence over approximately 30 (thirty) $4-\mathrm{km}^{2}$ sites, or by reliably measuring its relative abundance in just 3 (three) $4-\mathrm{km}^{2}$. In this case it would be much wiser to monitor the status of the population by the simpler and qualitative wider scale presence/ absence method, combining the data to produce a semi-quantitative frequency of occurrence.

Another consideration is whether to use direct or indirect measure of the attribute. A direct measure involves making measurements of the attribute itself (e.g. counting the number of livestock present). An indirect (surrogate) measure involves measuring a related variable, which is used to infer the status of the attribute being monitored (e.g. counting dung as an index of the number of livestock present). Such measurements are described as an index (in this case of population size). An index of population size is also obtained from direct sampling of a subset of a total population. For example, male pheasants can be counted by their calls, but this does not offer an index of total population size, since one cannot be sure of the numbers of females.

Finally it must be remembered that the selected method must produce a measurement that is consistent with the objective for each feature and its attributes. For example, the objective for species composition in a forest stand might be "70-90\% of trees of Quercus species". This could be measured by simply calculating the frequency of Quercus species encountered along a number of randomly located line transects (see Table 4.3). However, if the objective specifies a required density of Quercus trees then quantitative estimates of Quercus density on continuous scales of measurement are required using a different method. It is therefore important to consider the potential methods and costs of measuring the achievement of an objective when setting that objective (see Section 3.6). Thus if a method cannot be found that can adequately measure achievement of the objective with the resources (time, money, expertise) that are available, then it may be necessary to modify the objective. 


\subsubsection{Use a method that measures attributes across an appropriate range of conditions}

It is essential that the method is suitable for the range of conditions over which it is to be used. For example, a forest bird survey method must be appropriate for use across the range of forest types that are being monitored, e.g. from open forest to dense forest.

Within the ACA it is particularly important that methods can be applied across a wide range of terrains and altitudes. Methods that cannot be used on steep slopes will be of very little use!

\subsubsection{Use methods that measure appropriate degrees of change}

Time may be wasted if a method is used that is very precise when only large changes need to be detected. For example, it is not necessary to use complicated methods for measuring tree height to the nearest centimetre if the objective relates to ensuring $20 \%$ of trees are above $10 \mathrm{~m}$ height.

\subsubsection{Use a method with acceptable and controllable bias}

\section{What is bias?}

Bias is a systematic source of error that results in under- or over-estimation of the attribute being measured. For example a survey methodology may result in half of the individuals of a species being routinely overlooked, and as a result population estimates from the survey will always be half of the true value. Thus, bias causes estimates to be inaccurate. Methods free of bias are said to be accurate, but completely bias free methods are always impossible to obtain.

\section{Sources of bias}

Bias may arise from several sources in a study, including:

\section{Observer}

- Incorrect identification of species.

- Failing to detect and count all individuals of a particular species being monitored.

- Different observers may record identical observations in dissimilar ways.

- Differences in expertise between observers.

- Variation in observer effort (e.g. speed of assessment).

\section{Location}

- Studying a species only where it is common introduces bias; if areas where it is rare have been ignored the full dispersion of the species will not be understood.

a Using a small subjectively selected sample area when the site being studied is not homogenous will introduce bias.

\section{Habitat differences}

- Variation in the detectability of species in different habitats.

- Inabilities to access some habitats or areas.

\section{Species differences}

- Variation in detectability between species.

a Some species may be more easily identifiable than others. 


\section{Time related sources}

- The time of year (or day) when a survey is carried out can affect the results.

\section{Weather}

- Weather may affect the activity of animals and therefore their detectability (e.g. many birds stop singing in wet weather, and vultures and other raptors will not fly in bad weather when there are no thermals to enable soaring)

- Bad weather can reduce an observer's ability to see and hear (e.g. it is difficult to hear singing birds in windy weather)

- Inclement weather affects observers' concentration, as well as variation between different observers' capacity for working under difficult conditions can introduce a bias.

\section{Dealing with bias}

Awareness of such potential sources of bias when planning a monitoring programme can help avoid future analytical problems. Hill et al. (in press) suggest that there are three ways of combating bias:

1. Anticipated sources of bias can be reduced or controlled by:

- Using the same methods, observers and analysis etc., across years and sites. Ensuring that procedures are well documented helps to maintain consistency (see Section 4.10). If recording effort cannot be kept constant, the next best thing is to measure it, that way any observer bias can be more easily assessed.

- Checking that methodological assumptions are valid for the habitat or species you wish to study and for the period of time over which it is to be studied.

- Recording relevant weather conditions when surveying. Agree and record beforehand under which weather conditions work should be postponed.

- Agreeing and recording definitions (e.g. sample size, type, population unit etc.) beforehand.

- Calibrating observers against each other before and during monitoring. Introduce a system for verifying the data (perhaps by using a person unconnected with the study).

2. With careful design it is possible to avoid the problem by confining comparisons to results that have the same bias.

3. It may be possible, though difficult, to measure the bias. Measuring bias can be done only if the true value can be occasionally ascertained, which is normally unachievable. A separate experiment may be helpful - for example, one could compare the results obtained by different observers measuring the same population.

If the bias adversely affects the monitoring and cannot be adequately measured, controlled for or reduced, then an alternative method should be used. If it is not possible to find one method that provides an apparently unbiased estimate, use a number of different methods and compare the results, or change the objectives to match what is achievable.

\subsubsection{Methods for measuring habitat and species attributes}

The recommended uses and advantages and disadvantages of some methods for measuring attributes of habitats, vegetation and plants, and animals are outlined in Tables $4.2-4.4$ below. See the references listed in Box 4.1 for further guidance on the use of the methods and how to carry them out in practice. 
Table 4.2 Advantages and disadvantages of different habitat measurement methods

\begin{tabular}{|c|c|c|}
\hline $\begin{array}{l}\text { Method and } \\
\text { main uses }\end{array}$ & Advantages & Disadvantages \\
\hline $\begin{array}{l}\text { Satellite remote } \\
\text { sensing } \\
\text { Measurement } \\
\text { of habitat } \\
\text { extent \& major } \\
\text { changes in } \\
\text { composition }\end{array}$ & $\begin{array}{l}\text { A large area can be covered from a } \\
\text { single data source. Repeated samples } \\
\text { can be expected for the same area for } \\
\text { the lifetime of the satellite. } \\
\text { Satellite derived maps can be used to help } \\
\text { design a stratified sampling programme. } \\
\text { Data can be used to identify landscape } \\
\text { scale changes. } \\
\text { Archived data may be accessed to } \\
\text { provide a historical baseline prior to the } \\
\text { initiation of the monitoring programme. } \\
\text { Very useful for mountainous areas such } \\
\text { as the ACA where the terrain can limit } \\
\text { field surveys. }\end{array}$ & $\begin{array}{l}\text { The lifetime of the satellite may be } \\
\text { more limited than the lifetime of the } \\
\text { monitoring project, leading to potential } \\
\text { difficulties in comparing outputs from } \\
\text { original and replacement sensors. } \\
\text { Satellite scenes are likely to be } \\
\text { incomplete when clouds are present. } \\
\text { Error rates in habitat classification may } \\
\text { be unacceptable. } \\
\text { Range of expertise required is } \\
\text { broadened from ecology into GIS and } \\
\text { remote sensing }\end{array}$ \\
\hline $\begin{array}{l}\text { Aerial } \\
\text { photography } \\
\text { remote sensing } \\
\text { Measurement } \\
\text { of habitat } \\
\text { extent and } \\
\text { broad changes } \\
\text { in composition }\end{array}$ & $\begin{array}{l}\text { Provides a relatively quick assessment } \\
\text { of extent of broad habitat types and } \\
\text { broad changes in these. } \\
\text { Historical trends can be examined using } \\
\text { past photographs. } \\
\text { Allows quicker and more accurate } \\
\text { mapping than by ground survey. }\end{array}$ & $\begin{array}{l}\text { Good photographs are required for } \\
\text { accurate analysis. } \\
\text { Habitats cannot be classified in as much } \\
\text { detail as with ground surveys. } \\
\text { Some habitats can be hard to } \\
\text { distinguish on photographs, } \\
\text { necessitating field checking of results. } \\
\text { Area will be underestimated for slopes } \\
\text { unless three co-ordinates are used to } \\
\text { digitise maps. High altitude areas will } \\
\text { be overestimated relative to low ones. } \\
\text { Atmospheric/geometric corrections may } \\
\text { be more complex than with satellite } \\
\text { monitoring, as the aircraft is not in a } \\
\text { stable orbit. }\end{array}$ \\
\hline $\begin{array}{l}\text { Fixed point } \\
\text { photography } \\
\text { Records broad } \\
\text { changes in } \\
\text { habitat } \\
\text { structure }\end{array}$ & $\begin{array}{l}\text { Records a wide range of attributes of a } \\
\text { habitat and it is not necessary to } \\
\text { anticipate the changes that are likely to } \\
\text { take place. Therefore this method may } \\
\text { detect unexpected changes which are } \\
\text { unmeasured by other methods. } \\
\text { Quick and simple, and provides a visual } \\
\text { picture of change with time. } \\
\text { Better than aerial photographs for steep } \\
\text { slopes. }\end{array}$ & $\begin{array}{l}\text { Generally only gives broad indications } \\
\text { of change, which cannot easily be } \\
\text { quantified or tested by objective } \\
\text { statistical methods. }\end{array}$ \\
\hline
\end{tabular}


Table 4.3. Advantages and disadvantages of different methods for measuring vegetation and plant attributes

\begin{tabular}{|c|c|c|}
\hline $\begin{array}{l}\text { Method and } \\
\text { main uses }\end{array}$ & Advantages & Disadvantages \\
\hline $\begin{array}{l}\text { Quadrats } \\
\text { Measurement of } \\
\text { cover, density, } \\
\text { biomass and } \\
\text { frequency of } \\
\text { small shrubs, } \\
\text { herbs, grasses, } \\
\text { and lower } \\
\text { plants, and } \\
\text { associated } \\
\text { variables, e.g. } \\
\text { height. }\end{array}$ & $\begin{array}{l}\text { A very widely used and documented } \\
\text { method. } \\
\text { Cover values provide good descriptions } \\
\text { of the contribution that each species } \\
\text { makes to vegetation communities. } \\
\text { Frequency estimates are easier to } \\
\text { collect and more reliable than cover } \\
\text { values, but require more samples and } \\
\text { are affected by quadrat size. }\end{array}$ & $\begin{array}{l}\text { Recording all species present can be } \\
\text { time-consuming. } \\
\text { Inaccuracies in species identification } \\
\text { occur for difficult species. } \\
\text { Estimation of cover can vary } \\
\text { significantly between recorders. } \\
\text { Cover scales (e.g. Domin) are non- } \\
\text { linear and therefore values, even from } \\
\text { randomly located quadrats, can only be } \\
\text { analysed using less powerful non- } \\
\text { parametric tests. }\end{array}$ \\
\hline $\begin{array}{l}\text { Line transects } \\
\text { Measurement of } \\
\text { cover and } \\
\text { frequency of } \\
\text { trees, shrubs, } \\
\text { herbs and } \\
\text { grasses, and } \\
\text { associated } \\
\text { variables, e.g. } \\
\text { height. }\end{array}$ & $\begin{array}{l}\text { Often simpler to use than quadrats, } \\
\text { especially in sparse or tall vegetation. } \\
\text { Easier to search thoroughly than } \\
\text { quadrats of the same area and quicker to } \\
\text { record. } \\
\text { Line point transects are useful for } \\
\text { measuring changes in total vegetation } \\
\text { cover, but accuracy depends on the } \\
\text { length of line and number of points } \\
\text { used per line. } \\
\text { Generally much faster method to } \\
\text { employ than quadrats or transects. }\end{array}$ & $\begin{array}{l}\text { Transects are often not suitable for } \\
\text { measuring cover of individual species } \\
\text { where plants are closely intermingled } \\
\text { and vegetation type boundaries are not } \\
\text { distinct. } \\
\text { Long transect lines produce under- } \\
\text { estimates of species cover when points } \\
\text { are widely spaced. However, estimates } \\
\text { of total cover are unaffected by length } \\
\text { of line } \\
\text { If species are at very low density it may } \\
\text { take a long time to locate the nearest } \\
\text { individual. }\end{array}$ \\
\hline $\begin{array}{l}\text { Plot-less } \\
\text { techniques } \\
\text { Measurement of } \\
\text { density of tree } \\
\text { density in } \\
\text { forests, and } \\
\text { associated } \\
\text { variables, e.g. } \\
\text { mean girth. }\end{array}$ & Little equipment is required. & $\begin{array}{l}\text { When surveying areas with high species } \\
\text { diversity, the time taken when } \\
\text { measuring separate species will } \\
\text { increase. } \\
\text { The method contains inherent bias due } \\
\text { to the non-random selection of trees. }\end{array}$ \\
\hline
\end{tabular}


Table 4.4. Advantages and disadvantages of different methods for measuring animal population attributes

\begin{tabular}{|c|c|c|}
\hline $\begin{array}{l}\text { Method and } \\
\text { main uses }\end{array}$ & Advantages & Disadvantages \\
\hline $\begin{array}{l}\text { Recording } \\
\text { incidental } \\
\text { observations } \\
\text { Establishment } \\
\text { of presence of } \\
\text { species, crude } \\
\text { estimation of } \\
\text { range if } \\
\text { sufficient } \\
\text { records. }\end{array}$ & $\begin{array}{l}\text { Collects records on rare or rarely } \\
\text { encountered species that cannot in } \\
\text { practice be monitored by other means. } \\
\text { Simple method which can involve all } \\
\text { staff and local communities. }\end{array}$ & $\begin{array}{l}\text { Observation and recording effort varies, } \\
\text { and therefore data cannot be easily } \\
\text { analysed for trends etc, although staff } \\
\text { activities data can be assessed in } \\
\text { relation to effort if properly recorded. } \\
\text { Reliability of records variable and } \\
\text { difficult to assess. } \\
\text { Variable biases. }\end{array}$ \\
\hline $\begin{array}{l}\text { Timed searches } \\
\text { Establishment } \\
\text { of presence of } \\
\text { species in a } \\
\text { sample area, } \\
\text { and estimation } \\
\text { of relative } \\
\text { abundance. }\end{array}$ & $\begin{array}{l}\text { Relatively simple efficient method. } \\
\text { Useful for scarce species or species that } \\
\text { are difficult to detect. } \\
\text { More practical than transects in difficult } \\
\text { terrain. }\end{array}$ & $\begin{array}{l}\text { Normally only crude indices of } \\
\text { abundance. } \\
\text { Likely to be biased according to } \\
\text { observer ability and effort. } \\
\text { Detection biases cannot be easily } \\
\text { controlled for. }\end{array}$ \\
\hline $\begin{array}{l}\text { Point counts } \\
\text { Measurement of } \\
\text { relative } \\
\text { abundance (or } \\
\text { density) of } \\
\text { highly visible } \\
\text { or vocal species } \\
\text { (usually birds). }\end{array}$ & 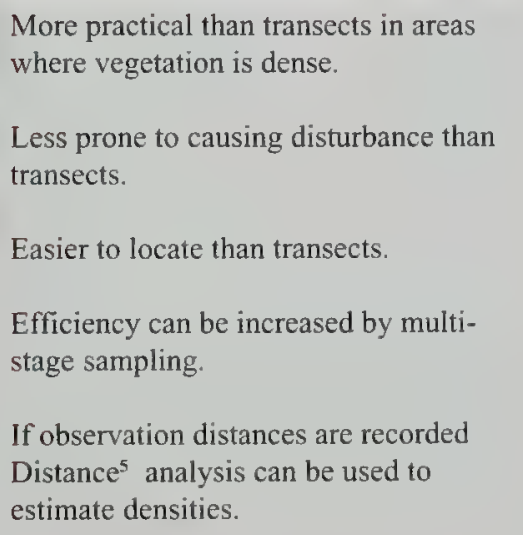 & $\begin{array}{l}\text { Less efficient than transects, } \\
\text { particularly for less detectable species. } \\
\text { More observations are by song / call } \\
\text { than by transects and therefore } \\
\text { considerable experience is required. } \\
\text { Distance analysis more prone to bias if } \\
\text { used than transects. }\end{array}$ \\
\hline $\begin{array}{l}\text { Transects } \\
\text { Measurement of } \\
\text { relative } \\
\text { abundance (or } \\
\text { density) of a } \\
\text { variety of birds, } \\
\text { mammals and } \\
\text { other animals. }\end{array}$ & $\begin{array}{l}\text { Relatively efficient compared to point- } \\
\text { counts especially where birds are at low } \\
\text { densities. } \\
\text { If observation distances are recorded } \\
\text { Distance analysis can be used to } \\
\text { estimate densities. } \\
\text { Good for open habitats. }\end{array}$ & $\begin{array}{l}\text { Often difficult to randomly allocate } \\
\text { routes. } \\
\text { Difficult in dense vegetation and steep } \\
\text { terrain. } \\
\text { Movement may scare shy species. } \\
\text { Strip transect densities may be } \\
\text { unreliable. }\end{array}$ \\
\hline
\end{tabular}

'See Buckland et al. (2001) for details of Distance sampling techniques, but these are normally too complicated and time consuming for routine monitoring purposes. 


\subsection{Establish the appropriate time to carry out surveys}

The appropriate time for carrying out monitoring will vary according to the feature and attribute being measured. For example, most breeding bird monitoring is best carried out early in the breeding season when singing is at its greatest, whilst Snow Leopard surveys are best carried from January to April when they are mating and marking their home ranges most intensively.

The time of day is also often a vitally important factor to take into account, for example pheasants generally only call for a short period around dawn, and therefore surveys carried out an hour or so later will be ineffective. In contrast, surveys of soaring vultures must be carried out later in the day when the sun is up and thermals have been created. Knowing the activity patterns of your study animals is therefore important, and it is essential that if there are strong diurnal activity patterns that survey times are standardised with respect to these.

It is particularly important that repeat surveys in subsequent years are carried out at the same time of year each year, unless seasonal cycles are being investigated, and at the same time of day. Serious bias may occur if surveys are carried out at different times.

\subsection{Devise a sampling scheme}

\subsubsection{Decide if sampling is needed}

In some situations it may be possible to make a complete assessment of the whole feature within your target area. For example it may be possible to reliably measure the full extent of a habitat feature by aerial photography. Or it may be possible to carry out a complete census of a rare species by counting all individuals if the species is easily detectable and highly localised (e.g. vultures breeding at cliff nesting sites). However, care should be taken, as you may be mistaken in believing that you have detected all occurrences of the species. This is particularly important when dealing with mobile species, because a decline in your monitored population may simply be due to their relocation to another site that you are unaware of. Thus a decline in a vulture breeding colony could be merely because they have moved to a new nesting site.

In practice, it is seldom possible, or even necessary, to establish the total population size of a species. Unless species are very rare, very conspicuous, and very localised, total counts will probably be too time-consuming and will produce biased results.

Generally, it is most efficient to assess samples of the feature and to extrapolate from the observations made in each sample to the whole feature (or that part covered by the sampling area). For the inferences that one draws about the whole to be valid, sampling must follow certain principles:

₫ Samples must be representative of the site.

- More than one sampling unit per habitat is required. This is known as replication.

Sampling enables the estimation of an attribute's value for a whole site, and also estimates the inherent uncertainty in this value due to having only studied part of the site 
(or population). For example, the area of a monitoring unit (e.g. a hill) covered by a Rhododendron may be estimated by calculating the mean area of Rhododendron in a sample of $10 \mathrm{~m} \mathrm{x} 10 \mathrm{~m}$ plots and multiplying this figure by the size of the site in square metres. The uncertainty in this estimate can be measured by the standard deviation of the estimate, or by confidence intervals (see Elzinga 2001) or other references listed in Box 4.1 for details, and Section 4.15 for advice on statistical analysis).

When designing a new monitoring programme it is advisable to design your sampling strategy to your specific needs. The design of a sampling strategy is a particularly important stage in the development of an effective monitoring programme and should, therefore, be carried out carefully according to the key steps summarised in Figure 4.4. Sampling should be designed for each feature's attribute, taking into account the method being used to measure it, the inherent properties (e.g. relative density) and variability of the attribute (if known), the required precision of measurement, and the time and costs of sampling. The design of a sampling strategy will also need to take into account the size of the sampling unit being used (e.g. a $2 \mathrm{~m}$ by $2 \mathrm{~m}$ quadrat). This will in turn depend on the species or habitat being sampled, the type of measurements being made and the method used for sampling.

Once the required sampling has been identified for each feature and attribute, then these can be combined to create an overall sampling programme, including combined data collection where appropriate. For example one might use the same quadrat samples to collect information on bare soil, vegetation height, vegetation species richness and the presence of selected species.

\section{Plate 4.3 Himalayan Tahr in the Sagarmatha National Park, Nepal}

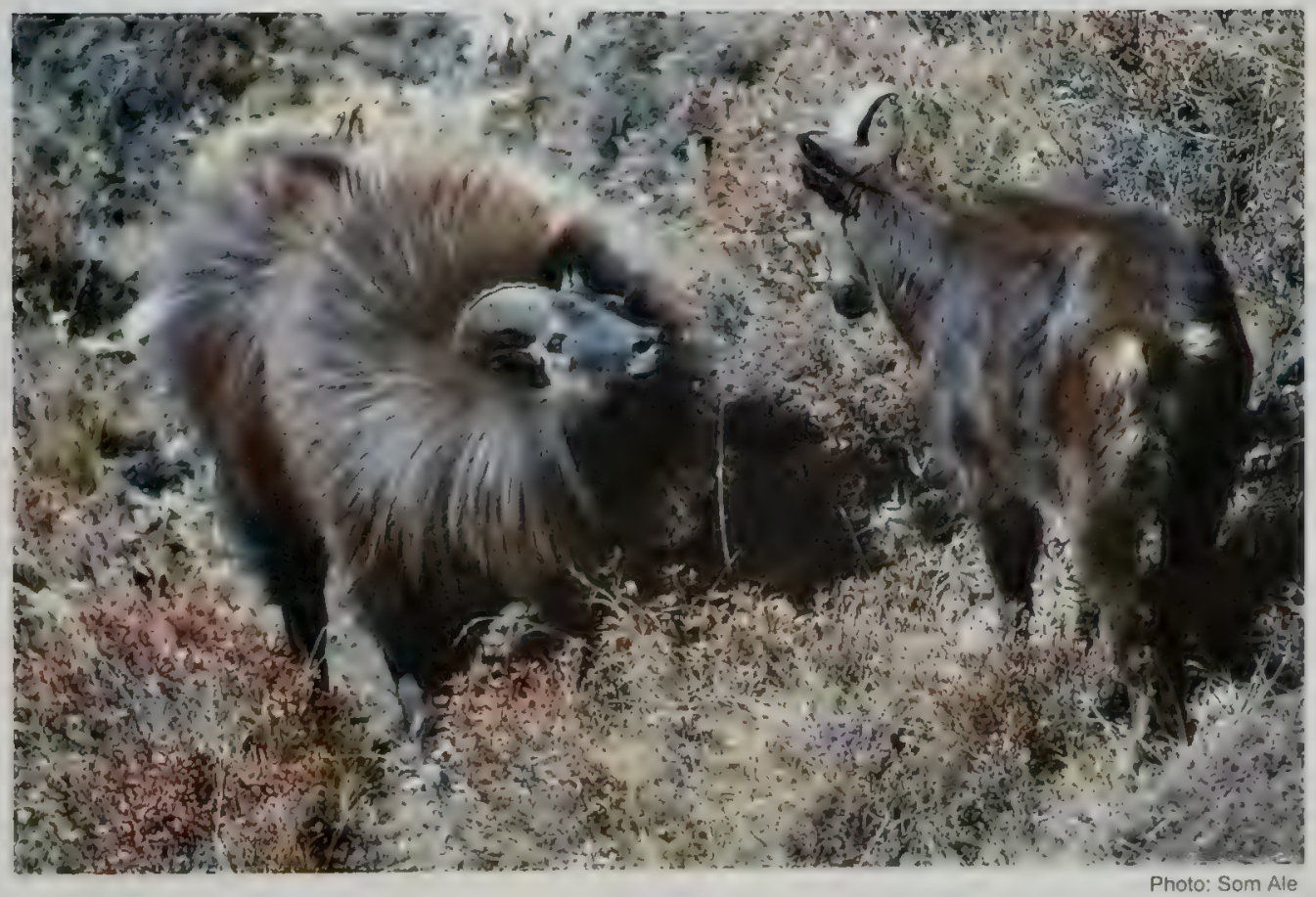


Figure 4.4 Designing a sampling scheme

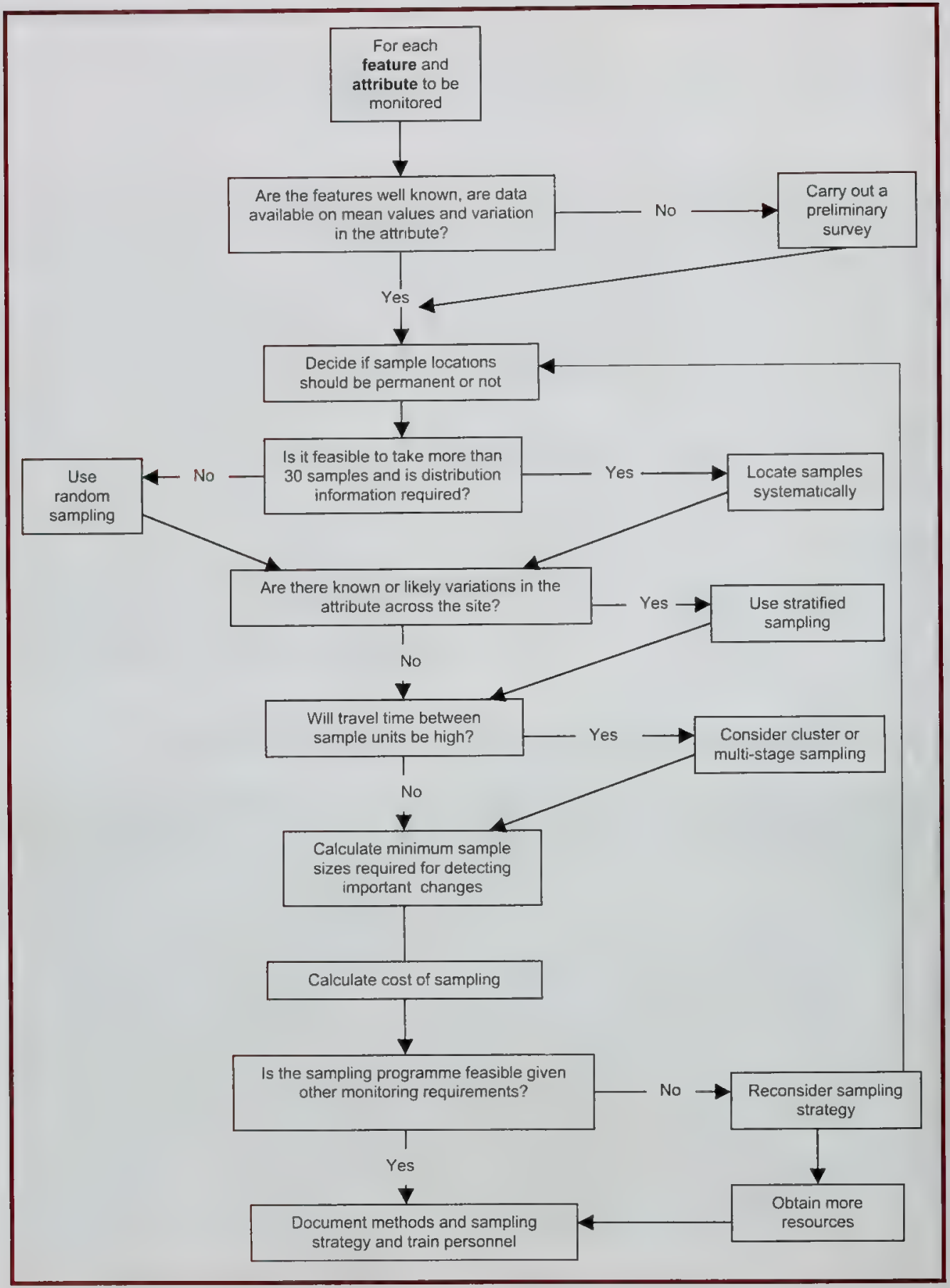




\subsubsection{Decide if sample locations should be permanent or temporary?}

\section{Advantages of permanent plots}

Permanent sample locations can provide a good approach for reducing variability when temporal changes are to be monitored. Therefore, they increase the statistical power of the monitoring, which means that fewer samples are needed to obtain a desired level of precision and hence detect an important change. If permanent plots tend to change in similar ways, then any changes documented are more likely to be real than due to random variation between samples. For example, if mean species richness over 20 temporary randomly located quadrats is 20 in one year and 10 in a subsequent survey, this may not be due to a real change in overall species richness, but could instead be caused by the chance location of quadrats in the first survey in richer parts of the site. However, if the repeated observations were made at the same locations and the locations are representative of the site, we can be more certain that species richness is declining on the site as a whole.

The usefulness of permanent plots varies depending on the degree of correlation between two successive measurements. Permanent sampling will be most advantageous for monitoring when there is a high degree of correlation between sampling-unit values between two periods. This is most likely to occur with long-lived plants (e.g. trees, shrubs, some perennials and lichens) and large territorial mammals, and least likely with erratic, transient or mobile populations such as some annual plants, small mammals and insects.

See Elzinga (2001) or other sources of statistical information listed in Tables 4.7 and 4.8 for advice on analysis of permanent plot data.

\section{Disadvantages of permanent plots}

There are some significant disadvantages with permanent plots that should be considered. Most importantly, marking and relocating permanent sample locations can be difficult and time-consuming. This may offset any advantage from additional precision if observations from non-permanent samples can be obtained much more quickly.

Repeatedly surveying the same locations may alter or damage the attribute being monitored or its surroundings, e.g. by trampling. Apart from the potential unacceptability of such damage, this may cause the samples to become unrepresentative of the site as a whole. However, this is more likely to be a problem for intensive scientific studies where frequent measurements are necessary, rather than routine protected area monitoring.

If the use of permanent sampling results in very few samples being taken, then additional practical problems may result. If there are only a few plots, then these may become unrepresentative of the whole study area (assuming that they were representative initially) as a result of chance events with a different effect on the plot to that on the rest of the area. Such events may also have permanent or long-lasting effects, as successive changes at one point tend to be correlated. Therefore, any recorded changes will not reflect the true pattern of change over the area. This difficulty (termed autocorrelation) can be overcome by recording a second set of samples at the end of the first monitoring period, which are used to estimate changes in the second period and so on. Samples A are enumerated on the first survey occasion, samples A and B on the second, samples B and C on the third and so on. 
Permanent sample locations may also be effectively lost due to unforeseeable events such as permanent or long-term flooding of part of the site, or the growth of trees over long time periods. Human encroachment may also lead to loss of samples, particularly in developing countries with expanding populations and agricultural landuse. For example this may lead to the loss of forest plots as forests are cleared for housing or slash and burn cultivation. This problem can be alleviated by recording 'spare' samples, though this may also reduce the advantage of the approach compared with temporary sampling.

\subsubsection{Choose an appropriate means of distributing samples}

According to Elzinga et al. (2001), there are three requirements that must be met with respect to the positioning of sampling units in the sample population:

1. Some type of random, unbiased sampling method must be used;

2. The sampling units must be positioned to achieve good interspersion throughout the populations; and

3. The sampling units must be independent of each other.

Of particular importance is the random selection of sampling units. If this is not done, then you cannot make any statistical inferences from your results. Selection of samples by judgement (or preferential sampling) should be strictly avoided.

The advantages and disadvantages of some means of sampling are described below in Table 4.5 and illustrations of different sample designs are given in Figure 4.5.

\section{Table 4.5. Summary of the advantages and disadvantages of different approaches to sample distribution}

\begin{tabular}{|c|c|}
\hline Method and main uses & Disadvantages \\
\hline $\begin{array}{l}\text { Simple random sampling } \\
\text { Requires minimum knowledge of a population } \\
\text { in advance. } \\
\text { Easy to analyse data and compute errors. } \\
\text { Selecting sample units is quicker and easier than } \\
\text { for other random designs. }\end{array}$ & $\begin{array}{l}\text { Collection of sample observations can be time- } \\
\text { consuming. } \\
\text { Often larger errors for a given sample size than } \\
\text { with systematic sampling. } \\
\text { Estimates will be less precise on heterogeneous } \\
\text { sites than with stratified sampling. } \\
\text { Travel time between sample units can be high. }\end{array}$ \\
\hline $\begin{array}{l}\text { Systematic sampling (regular) } \\
\text { If the population or attribute is ordered with } \\
\text { respect to some pertinent variable such as an } \\
\text { altitudinal or moisture gradient, there is a } \\
\text { stratification effect, which reduces variability } \\
\text { compared with random sampling. } \\
\text { Calculation of sample positions and location in } \\
\text { the field is relatively easy and efficient. }\end{array}$ & $\begin{array}{l}\text { If sampling interval is correlated with a periodic } \\
\text { feature in the habitat, bias may be introduced. } \\
\text { Strictly speaking, statistical tests are not valid, } \\
\text { though in practice conclusions are unlikely to be } \\
\text { affected if there are more than } 30 \text { potential sample } \\
\text { (Cont.) }\end{array}$ \\
\hline
\end{tabular}




\section{Table 4.5. Summary of the advantages and disadvantages of different approaches to sample distribution (cont.)}

Provides an efficient means of mapping distribution and calculating abundance at the same time.

\section{Stratified random}

Ensures that all the main habitat types present on a site will be sampled (if these are used to define strata).

Characteristics of each stratum can be measured and comparisons between them can be made.

Greater precision is obtained for each stratum and for overall mean estimates if strata are homogeneous.

\section{Restricted random sampling}

Like systematic sampling it results in good interspersion of samples, but is more robust when the number of possible samples is few.

Like stratified random sampling in that the plot is subdivided (but in this case with arbitrary divisions and 1 sample in each). Results in more precise estimates than simple random sampling.

\section{Multi-level (plots within plots)}

Can reduce sampling times thus increasing efficiency.

Can avoid loss of precision caused by combining individual sampling units which occurs with cluster sampling.

Useful for sites which are heterogeneous at small spatial scales locations. (e.g. a $1 \mathrm{~m}^{2}$ sample could be located in 36 locations in a sampling grid of $6 \mathrm{~m} \times 6 \mathrm{~m}$ ).

If strata have not been identified prior to monitoring, preparation can be time-consuming.

The most appropriate stratification for a site at one time may have changed when repeat surveys are carried out. Monitoring efficiency may therefore also change.

Sampling units can occur side by side (like other forms of random sampling), and therefore systematic sampling is normally better if the potential number of systematic samples is large enough (i.e. more than 30 ).

Larger errors are obtained if secondary sample plots within each primary plot are highly correlated. 
Figure 4.5 Different types of sampling strategy

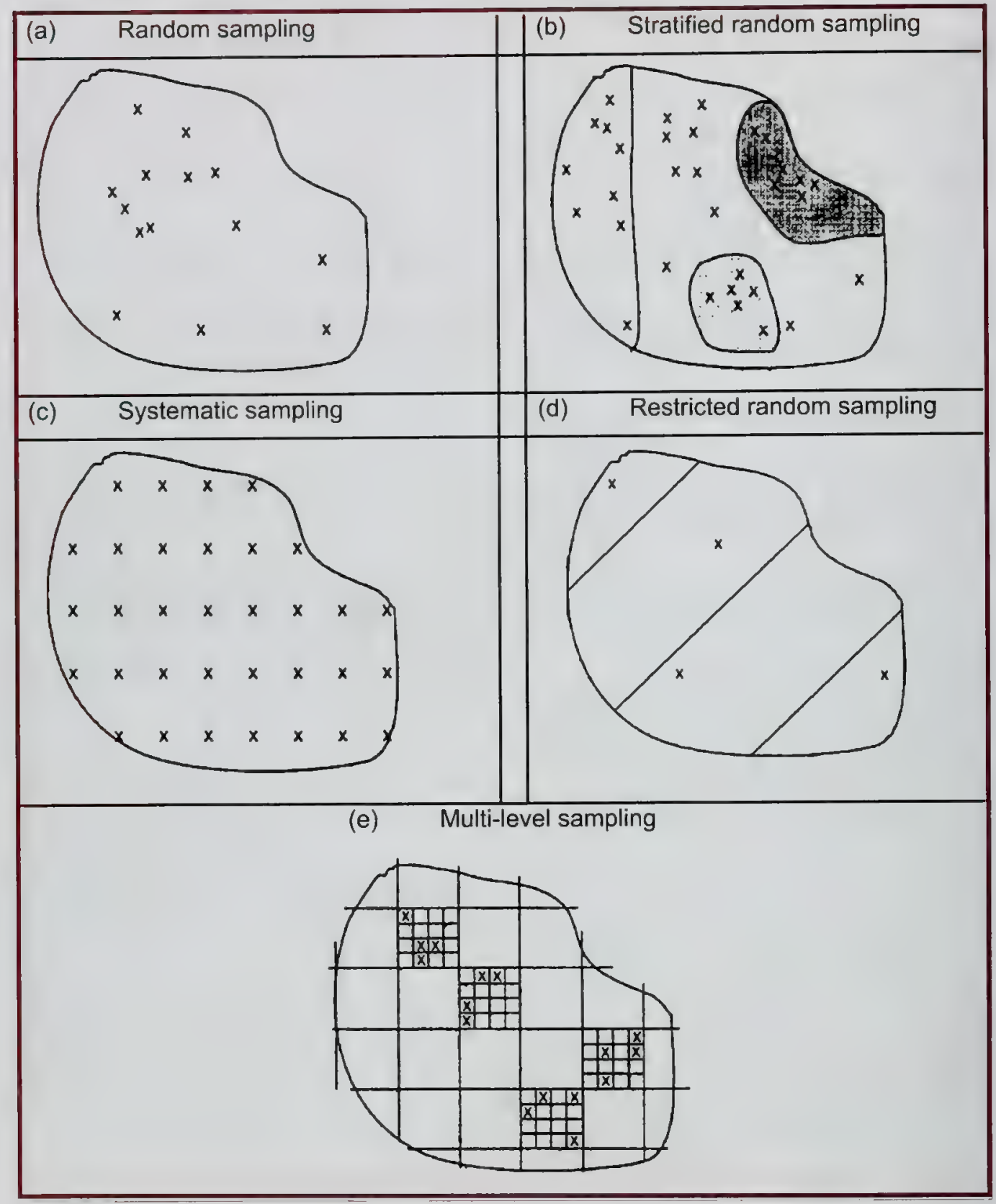

(a) Random sampling: samples taken randomly from the whole sampling area (b) Stratified random sampling: sample area divided into strata and random samples taken in each stratum (c) Systematic sampling: samples taken at regular intenvals (d) Restricted random sampling: one sample randomly locuted in each arbitorily defined sub-section of the sampling area and (e) Mutli-level sampling: major units (large squares) chosen randomly and minor units $(x)$ sampled randomily from each, major units may also be trasects. 


\subsubsection{Estimate the number of samples that will be required to reliably establish if objectives are being met}

A key principle of sampling is that with increased sample size our uncertainty decreases regarding how closely the estimated population value reflects the true population value. Thus, we would expect that as more samples are taken the closer the estimated mean will be to the true value. Unfortunately, the greater the sample size the greater the amount of survey time required. Additional time is required to take the measurement at each sample location and to move between sample locations, and the latter time may be particularly substantial in large protected areas with difficult terrain (as in the ACA). Furthermore our precision (i.e. the closeness of the sample measurements to each other) in estimating the mean only increases slowly once we go beyond a few samples. Typically, precision increases only in proportion to the square root of the sample size. Hence, to double the precision obtained from ten sample units requires another 30 units.

So a balance is needed between limiting time in sampling and ensuring the estimate is adequate. But defining what is 'adequate' sampling is not easy because it depends on the reliability of information required, which in turn depends on the importance, objectives and condition of the feature being monitored. For example, if a key feature is very important and its population is believed to be close to the limit of what is viable, then a good, or precise, population estimate is likely to be required. In other situations, only a quick check may be needed to confirm that an objective is being met.

Because increasing the number of replicated samples increases the cost of monitoring, it is very useful to carry out pilot surveys. These pilots aim to assess the distribution and abundance of the species or habitat attributes being monitored, so that the amount of variation in each can be approximately calculated. This can help in designing the sampling system and establishing the number of samples required to achieve a desired level of precision or to detect a given level of change.

In general, measurements should be taken from at least five plots before any generalisations can be made about a population or habitat within the sample area. Even this low level of replication can improve the confidence with which the results can be regarded. For frequency data, it has been shown that with less than 50 samples, only very large changes are likely to be statistically significant, and 100 samples has been suggested as a minimum. Bonham (1989) suggests that for most purposes 25 quadrats randomly and temporarily located on 25 randomly and temporarily located transects should give satisfactory results within a homogenous plant community.

There are now sophisticated means of establishing the number of samples required to detect desired changes, using Power Analysis. This is beyond the scope of this guidance but further information on these approaches can be found in Thomas \& Krebs (1997). though more up to date information can be found on the Internet.

\subsection{Devise data recording forms and document methods}

\subsubsection{Design field data recording forms}

Once the method and sampling design has been chosen, field data sheets should be designed and tested. Specially designed forms encourage consistency and reduce unnecessary writing. 
Forms are easy to read and help ensure that all necessary data are collected and not forgotten. See Elzinga et al. (2001) for suggestions for standard information to include in forms.

It is vital that all relevant sections of survey forms are completed at the time of the survey, and checked immediately after. Do not leave form filling for later, as information may be forgotten or entries in field notebooks misinterpreted.

Where lots of data are being recorded relatively quickly, it may be advantageous to type the data directly into a hand-held data-logger, which may include a GPS, thus providing accurate spatial reference data for each record. However, such data loggers can be heavy and expensive. If a data logger is used, a database structure should be written which prompts the observer to enter the appropriate record. The advantage of this method is that a large data set can be downloaded directly to a computer via a cable.

\subsubsection{Document field methods as monitoring protocols}

It is essential that the monitoring methods are constant between surveys. Therefore, before the first survey is carried out, a monitoring protocol should be written describing in detail the methods to be used, so that everyone understands what is required, and the methods are kept consistent between observers. A monitoring protocol should be prepared for each feature to be monitored and should document each of the issues as listed in Box 4.3.

Example protocols for remote sensing of habitat extent, forest quality, forest bird assemblages, vultures and Snow Leopards are provided in section $8.2-8.6$ of these guidelines.

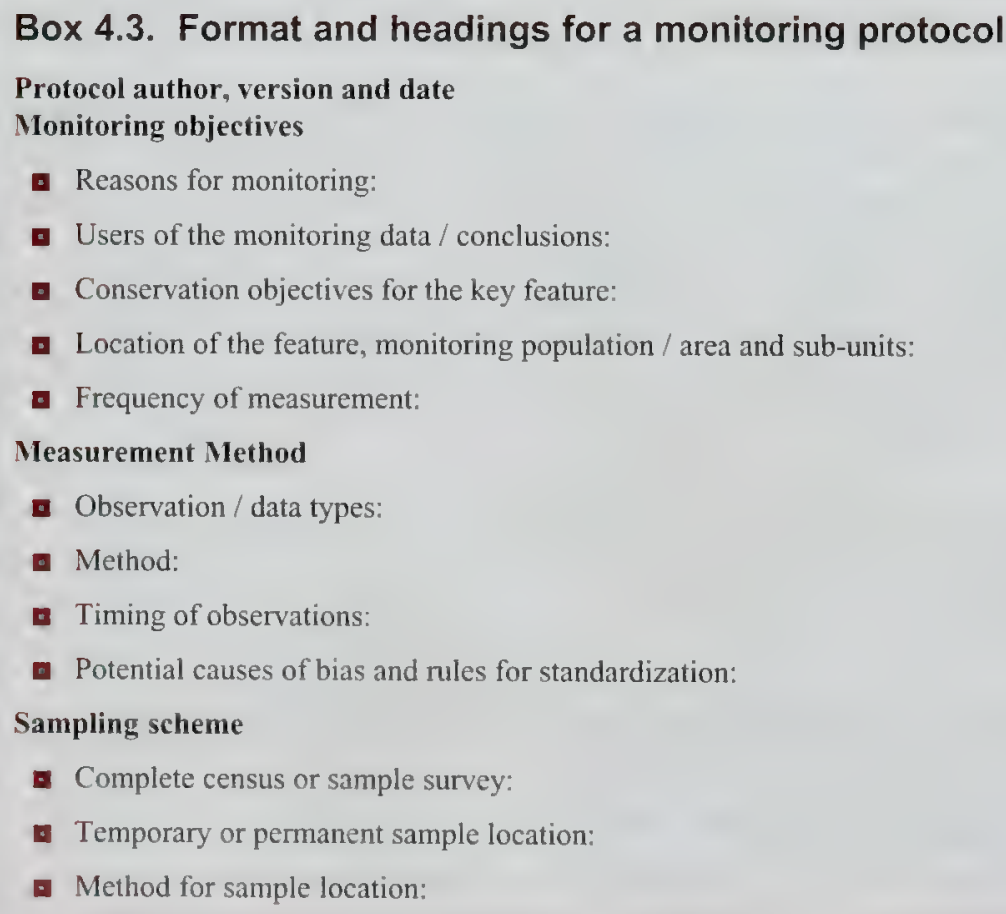




\section{Box 4.3. Format and headings for a monitoring protocol (cont.)}

- Number of samples:

Monitoring requirements \& organisation

Personnel responsible and time required:

- Experience / training necessary:

a Licence and access permission requirements:

a Equipment required:

- Data recording and storage

- Data analysis procedures

- Reporting format and procedures:

- Costs: capital (equipment) and annual recurrent (including staff time and travel etc)

\section{Health and safety}

a Any particular risks with carrying out the fieldwork, and requirements for ariy special equipment of measures to be taken to reduce risks.

\section{References}

Fieldwork can be dangerous and so before carrying out any such work a risk assessment should be carried out. Careful consideration must then be given to identifying safety precautions that reduce any identified risks to acceptable levels. All identified safety precautions should then be strictly followed.

Each protocol should then be followed as closely as possible in all subsequent surveys. However, if deviations from the protocol are necessary, then these should be recorded. Monitoring reports should ensure that the methods are written out in full and the original monitoring protocol placed in an appendix. Deviations from the protocol should be reported and the implications for the results and interpretation of the monitoring discussed.

Even when monitoring methods are very carefully documented, surveys should be repeated by the same observers as much as possible. There is no doubt that the accuracy of interpretation is considerably enhanced when one observer repeats the surveys over a long period of time. If this can't be done, then another option is to use many observers and to randomly allocate sampling to them, so that systematic biases are avoided.

\subsection{Test methods}

It is highly recommended that you test your proposed monitoring methods and sampling strategy once you have drafted your protocols. Data from such pilot surveys enable observers to become familiar with the practicalities of using the method in relation to the terrain, the physical structure of the habitat and the behaviour of the study species. It may also provide an initial assessment of how close biodiversity features are to their conservation objectives (which may influence the effort needed to monitor them fully) and an estimate of the degree of variation present in each feature's attributes. This information is invaluable for finalising the optimal type of sampling, the distribution of samples, the required level of precision and the number of samples required to achieve this. Monitoring protocols should then be revised according to the findings and any methodological changes that need to be made. 
Testing of monitoring methods should also include trials of the statistical analysis of the data (see Section 4.15 for selection of methods). These trials would ideally be with data from pilot surveys, but even the use of invented data is better than no testing of the statistical methods selected. The statistical method for analysing the data from monitoring should always be determined before the data is collected, as this will influence the survey design. If surveys are to be carried out by different observers then it is important to check the repeatability of the method where feasible. This can be tested by having one observer repeat a survey immediately after another observer, or by the same observer conducting duplicate counts. The results of such tests may indicate differences in the ability of surveyors, such as in species identification, which might then be overcome by training, etc. The results of method assessments can also be incorporated into statistical tests. Confidence limits and variance can be calculated on the variation in total counts or mean values in order to separate variation caused by observer bias from all other variation. If major discrepancies are found between two of these calibrating surveys, the underlying cause should be identified and corrected if possible.

Although pilot trials may be time-consuming, they save time and resources in the long term, especially in a large area such as the ACA where monitoring is likely to require considerable travel between sites. In such cases, sampling needs to be as efficient as possible.

\subsection{Review the monitoring programme in relation to available long-term resources}

Once all the monitoring requirements have been identified, and methods and sampling approaches devised to meet them, then the whole cost of the programme and staff time requirements should be assessed. The assessment should take a long-term view of the requirements for monitoring and available resources, including likely year-to-year variations in monitoring needs and budgets. A poor monitoring design is one in which the monitoring effort changes from year to year, or monitoring is dropped because of a lack of resources. It is a common mistake for new monitoring programmes to be too ambitious.

It is therefore vital that the overall long-term requirements for all the proposed monitoring is reviewed in relation to available resources (including funding, equipment, staff time, staff expertise and the capacity for local participation) before finalising the programme and preparing a detailed monitoring plan. The review must take into account training needs for staff and other personnel (e.g. contracted surveyors or local participants). As a minimum, it is essential that all monitoring personnel are familiar with the habitat, study species and survey methods required. The correct identification of target species may require specialist personnel even if the methods themselves are straightforward. If the monitoring involves several people, they should all be trained to a minimum standard and recording techniques should be standardised, e.g. as part of a pilot study.

A careful evaluation of the equipment needed should also be undertaken during the review of the monitoring programme. All equipment needed for the monitoring programme should be available for its duration. If equipment is to be purchased, especially if this is expensive, detailed advice on its suitability for the monitoring plan should first be obtained. If it is found that the resources needed for a full monitoring programme exceed those available, then it will be necessary to seek more funds, and/or trim the monitoring programme in the 
least damaging way, e.g. by targeting monitoring as suggested in Box 4.2. However, it should be remembered that cutting back on monitoring may be a false economy, as monitoring may enable early management intervention which avoids very costly damage.

\subsection{Prepare a work plan}

\subsubsection{Agree responsibilities for the monitoring programme}

A complex monitoring programme requires careful coordination, to allow enable integrated data analysis and reporting. For example, key decisions need to be made on:

- who enters the data and is responsible for quality control

- who will manage the data

- who holds mastercopies - how is this related to versions of the database elsewhere?

- who has access to the final information

- who will analyse the data

- who will report on the results of the monitoring to users and managers

- who owns the data

a who has rights of use

It is therefore recommended that a monitoring manager / coordinator is appointed who has overall responsibility for deciding on these issues and coordinating the implementation of the overall programme. Key interactions between the coordinator and other members of staff, other organisations and stakeholders should also be identified, together with information flows. This can be usefully documented as a monitoring programme coordination system (as for example indicated in Figure 4.6). 
Figure 4.6. The coordination and data flow system for ACAP biodiversity monitoring

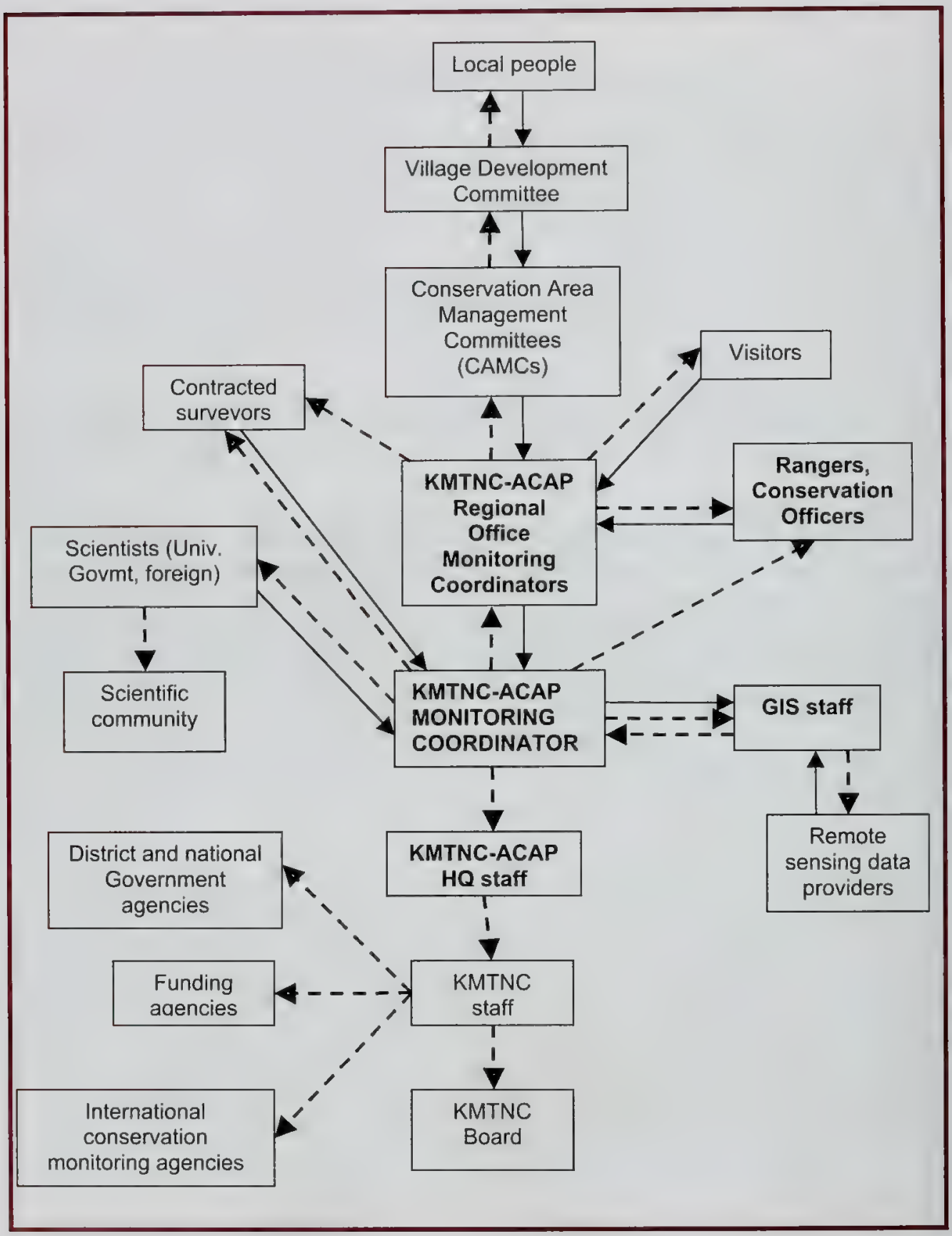

Key: Arrows indicate directions of information flow. Dashed lines indicate coordination guidance, analysed data and reports. Solid lines indicate primary data. ACAP staff are indicated in bold type. 


\subsubsection{Organise data collation and management}

Where feasible and appropriate monitoring data should be analysed and reported locally to increase local ownership of the monitoring programme. However, it will normally also be necessary to collate data for larger scale analysis and reporting, including for the protected area as a whole. Coordination will therefore be required to transfer data from the field, via a local office to a central database. Key coordination issues to be addressed will include:

a how will the data be transferred?

a who is responsible for data collation and transfer?

v in what format should the data be sent?

a what time schedule will be used, etc.?

Data management procedures should be documented and clearly communicated with everybody involved, making sure that responsibilities are clear.

When data arrive for central processing and storage there should be an initial quality check before any analysis is undertaken. This should look for common errors such as:

a missing data (i.e. gaps on data recording forms)

a recording errors (e.g. impossible or unlikely observations)

ש unreadable data entries

Data locations should also be checked by plotting the stated coordinates of the sampling sites on a map (e.g. using a GIS), so that incorrect coordinates might be apparent from a visual inspection of this map. Sometimes mistakes can best be detected by an expert. assessing the plausibility of results. Any detected errors and inconsistencies should be queried immediately with the original data providers (preferably the field workers themselves), who should keep a copy of any revised data themselves. Such quality control procedures will be the basis for ensuring scientifically sound and reliable outcomes of the monitoring programme.

Requirements for data storage should be addressed when the design of field forms and the design of the central database are being done. This will allow for an easy transfer from data recorded on paper to any electronic system, and help prevent mistakes. The use of standardized and widely recognised recording forms and coding systems (see Section 5.4) will greatly assist sharing data with others.

\subsubsection{Develop and agree a monitoring workplan}

A monitoring workplan should be developed, which summarises all the actions in the monitoring programme. There may be more than one monitoring action for each feature and attribute being monitored. For example, two methods to monitor wood collection for fuel are specified in Table 4.6. The work plan lists for each action its conservation or management objective, where the monitoring is to be undertaken. when, its costs. who is to carry it out, and what protocol is to be used (see Box 4.3). A section of a hypothetical workplan for ACAP is illustrated in Table 4.6. 


\begin{tabular}{|c|c|c|c|c|c|c|c|}
\hline 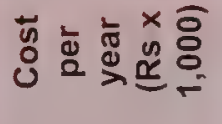 & $\underset{\infty}{8}$ & 8 & 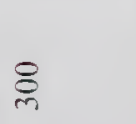 & $\begin{array}{l}8 \\
\stackrel{8}{*}\end{array}$ & $\frac{8}{7}$ & 点 & 8 \\
\hline $\begin{array}{l}\frac{2}{0} \\
\frac{0}{n} \\
\frac{c}{0} \\
\frac{0}{0} \\
0 \\
\square\end{array}$ & 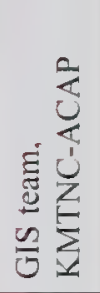 & 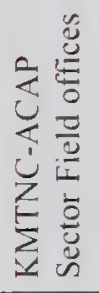 & 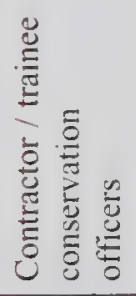 & 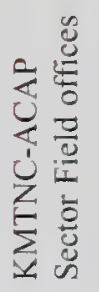 & 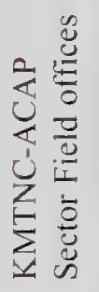 & 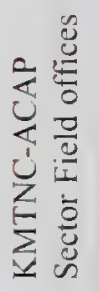 & 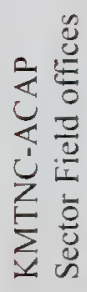 \\
\hline 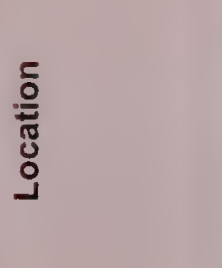 & $\begin{array}{l}\longleftarrow \\
\equiv \\
\bar{z}\end{array}$ & 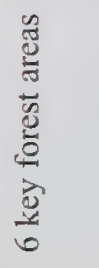 & 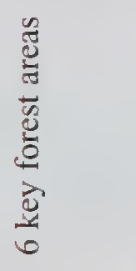 & 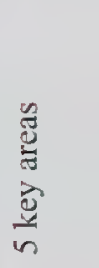 & 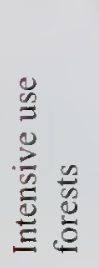 & $\sum^{\infty}$ & 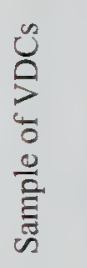 \\
\hline 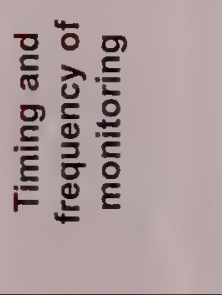 & 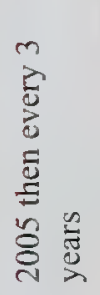 & 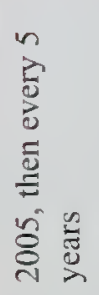 & 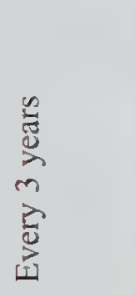 & 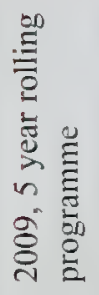 & 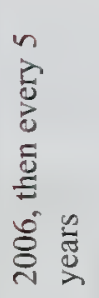 & 吢 & 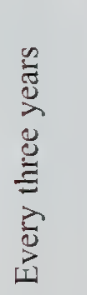 \\
\hline 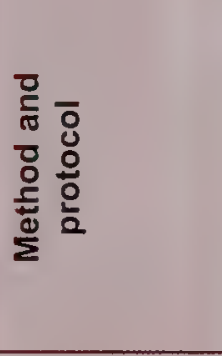 & 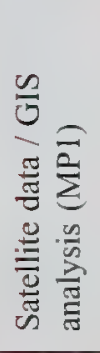 & 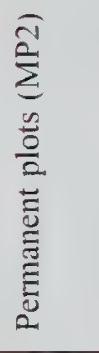 & 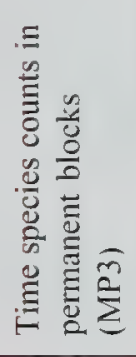 & 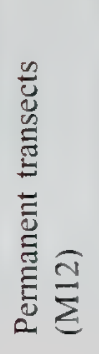 & 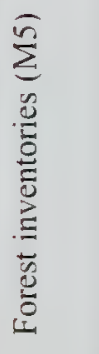 & 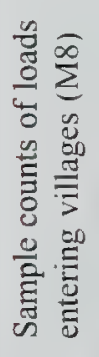 & $\begin{array}{l}\sum_{0}^{\infty} \\
\sum_{0}^{\infty} \\
\sum^{\infty} \\
\Xi\end{array}$ \\
\hline 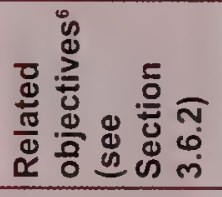 & $\bar{\omega}$ & 虫 & $\frac{U}{n}$ & $\bar{\omega}$ & 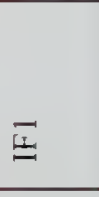 & $\bar{z}$ & $\bar{i}$ \\
\hline 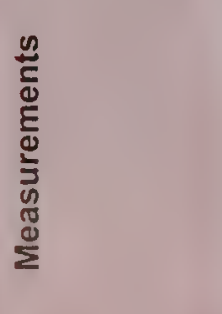 & 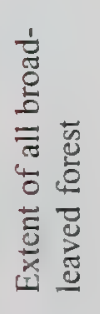 & 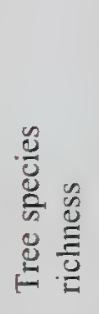 & 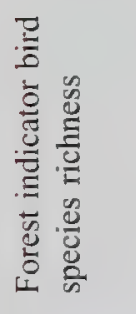 & 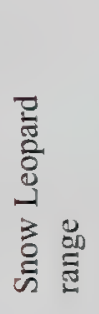 & 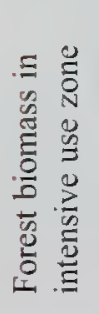 & 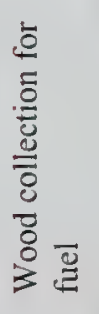 & 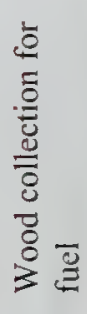 \\
\hline$\stackrel{E}{\Phi}$ & $\bar{\Sigma}$ & $\stackrel{\mathcal{N}}{\Sigma}$ & $\sum_{\Sigma}^{J}$ & $\stackrel{\infty}{\Sigma}$ & $\stackrel{\vec{J}}{\Sigma}$ & $\stackrel{N}{\Sigma}$ & $\hat{\Sigma}$ \\
\hline
\end{tabular}




\subsection{Carry out necessary training}

The establishment and maintenance of a biodiversity monitoring programme requires a range of skills and so training is likely to be necessary. All training activities should be based on an assessment of individual needs and build on the existing experience of staff and collaborators.

The first step in the identification of training needs is to determine the staff. local community groups and contractors involved in assessment and monitoring, from the collection and processing of data through to its interpretation and use in management decisions (see Figures 3.1 and 4.1). Much of this information will have been defined in the process of defining the monitoring programme and the preparation of a work plan and the monitoring protocols. Figure 4.6 (The co-ordination and data flow system for ACAP biodiversity monitoring) can be used as a guide for this step. For each actor involved in biodiversity monitoring, a short description of their responsibilities and the skills required should be prepared. This description of the ideal situation then provides a basis for an assessment of the current skills that exist and the needs for training.

Training will require a combination of teaching basic concepts and practical exercises to consolidate the learning of the participants. It is important to explain how field work and data analysis will be used in decision-making. Once the monitoring programme has been established many of the training requirements and procedures will be standardised procedures, as defined by the monitoring protocols. Each training session should include an anonymous feedback form, so as to continue improving the effectiveness of training.

For a protected area where management is in conjunction with local communities, such as the ACA, training needs will include appropriate data collection and analysis with designated community members. This training should be very practical, with practice of all the stages from identifying monitoring sites and completing forms to the interpretation of the results. The value of standardised procedures to allow the detection of change should be emphasised. On-going support should be provided to participatory monitoring work, to overcome any obstacles and maintain enthusiasm and standardised approaches.

\subsection{Analyse data}

\subsubsection{Key steps in statistical analysis}

Before starting an analysis, the steps are to:

1. Remind oneself of the question that the monitoring has been set up to answer (which should be linked to establishing whether or not a specific objective is being met).

2. Assess the quality and completeness of the available data, and fill data gaps where possible.

3. Choose an appropriate statistical method and package to conduct the analysis.

This section offers a quick guide in Table 4.7 to selecting appropriate significance tests, as this is often the most challenging decision in data analysis. 
Table 4.7. Choice of tests for different types of hypothesis

\begin{tabular}{|c|c|c|c|c|}
\hline Test for & $\begin{array}{c}\text { Date } \\
\text { description }\end{array}$ & $\begin{array}{l}\text { Parametric } \\
\text { alternative }\end{array}$ & $\begin{array}{c}\text { Non-parametric } \\
\text { alternative }\end{array}$ & Example use \\
\hline $\begin{array}{l}\text { Data normality for } \\
\text { single continuous } \\
\text { variables }\end{array}$ & Variable & - & $\begin{array}{l}\text { 2-tailed } \\
\text { Kolmogorov- } \\
\text { Smimov test }\end{array}$ & $\begin{array}{l}\text { Find out if a single } \\
\text { variable has a normal } \\
\text { distribution }\end{array}$ \\
\hline $\begin{array}{l}\text { Data of equal } \\
\text { variance }\end{array}$ & $\begin{array}{l}\text { Continuous } \\
\text { variable with } \\
\text { categories to } \\
\text { compare }\end{array}$ & - & Levene statistics & $\begin{array}{l}\text { Find out if a single } \\
\text { variable has equal variance }\end{array}$ \\
\hline $\begin{array}{l}\text { Linear correlation } \\
\text { between variables }\end{array}$ & $\begin{array}{l}\text { Two } \\
\text { continuous } \\
\text { variables }\end{array}$ & $\begin{array}{l}\text { Pearson's } \\
\text { correlation } \\
\text { coefficient }\end{array}$ & $\begin{array}{l}\text { Spearman's rho } \\
\text { coefficient }(+1 \text { to } \\
-1)\end{array}$ & $\begin{array}{l}\text { Find out whether spatial } \\
\text { variation in a species' } \\
\text { abundance is correlated } \\
\text { with an environmental } \\
\text { variable such as pH or } \\
\text { slope; or temporal } \\
\text { variation is correlated with } \\
\text { a change in a factor such } \\
\text { as grazing intensity }\end{array}$ \\
\hline $\begin{array}{l}\text { Difference between } \\
\text { two unrelated } \\
\text { groups }\end{array}$ & $\begin{array}{l}\text { Two } \\
\text { continuous } \\
\text { variables }\end{array}$ & $\begin{array}{l}\text { Unpaired t- } \\
\text { test }\end{array}$ & $\begin{array}{l}\text { Mann-Whitney } \\
\text { U-test }\end{array}$ & $\begin{array}{l}\text { Compare species richness } \\
\text { between two sets of } \\
\text { samples collected at } \\
\text { different times }\end{array}$ \\
\hline $\begin{array}{l}\text { Difference between } \\
\text { two related groups }\end{array}$ & $\begin{array}{l}\text { Two } \\
\text { continuous } \\
\text { variables for } \\
\text { same cases }\end{array}$ & Paired t-test & $\begin{array}{l}\text { Wilcoxon paired } \\
\text { signed-ranks test }\end{array}$ & $\begin{array}{l}\text { Compare relative } \\
\text { abundance in two sets of } \\
\text { permanently located } \\
\text { samples from two points } \\
\text { in time }\end{array}$ \\
\hline & $\begin{array}{l}\text { Binary } \\
\text { variable for } \\
\text { same cases }\end{array}$ & & McNemar's test & $\begin{array}{l}\text { Test significance of an } \\
\text { individual species being } \\
\text { present or not present in } \\
\text { two sets of permanently } \\
\text { located samples from two } \\
\text { points in time }\end{array}$ \\
\hline $\begin{array}{l}\text { Difference between } \\
\text { three or more } \\
\text { unrelated categories = } \\
\text { relationship between }\end{array}$ & $\begin{array}{l}\text { Continuous } \\
\text { variable with } \\
\text { nominal } \\
\text { categories } \\
\end{array}$ & $\begin{array}{l}\text { t-test ANOVA } \\
\text { (one-way) }\end{array}$ & $\begin{array}{l}\text { Kruskal-Wallis } \\
\text { H-test ANOVA }\end{array}$ & $\begin{array}{l}\text { Compare abundance at } \\
\text { three or more sites }\end{array}$ \\
\hline $\begin{array}{l}\text { continuous dependent } \\
\text { variable and one or } \\
\text { more categorical } \\
\text { independent variables }\end{array}$ & $\begin{array}{l}\text { Continuous } \\
\text { variable with } \\
\text { ordered } \\
\text { categories }\end{array}$ & $\begin{array}{l}\text { t-test ANOVA } \\
\text { (one-way) }\end{array}$ & $\begin{array}{l}\text { Jonckheere- } \\
\text { Terpstra } \\
\text { ANOVA }\end{array}$ & $\begin{array}{l}\text { Compare mortality or food } \\
\text { intake (continuous) with } \\
\text { age or size classes } \\
\text { (ordered) }\end{array}$ \\
\hline Time series & $\begin{array}{l}\text { Continuous } \\
\text { variable with } \\
\text { samples } \\
\text { through time }\end{array}$ & \multicolumn{2}{|c|}{$\begin{array}{l}\text { Time series analysis (e.g. via } \\
\text { regression) - or see 'Difference } \\
\text { between two related groups' to test for } \\
\text { difference between two time periods) }\end{array}$} & $\begin{array}{l}\text { Simulate future pattern } \\
\text { based on past history. }\end{array}$ \\
\hline
\end{tabular}




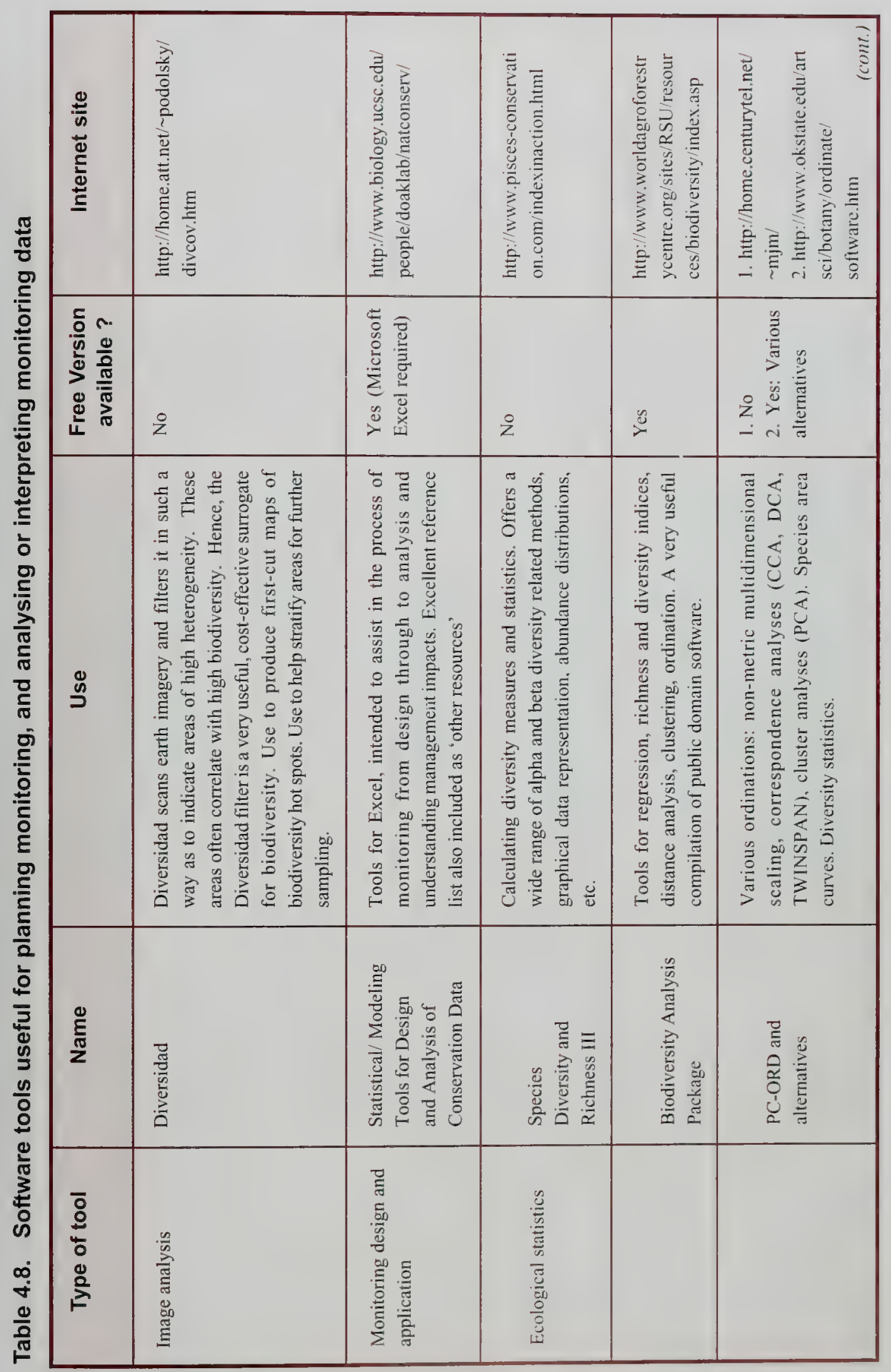




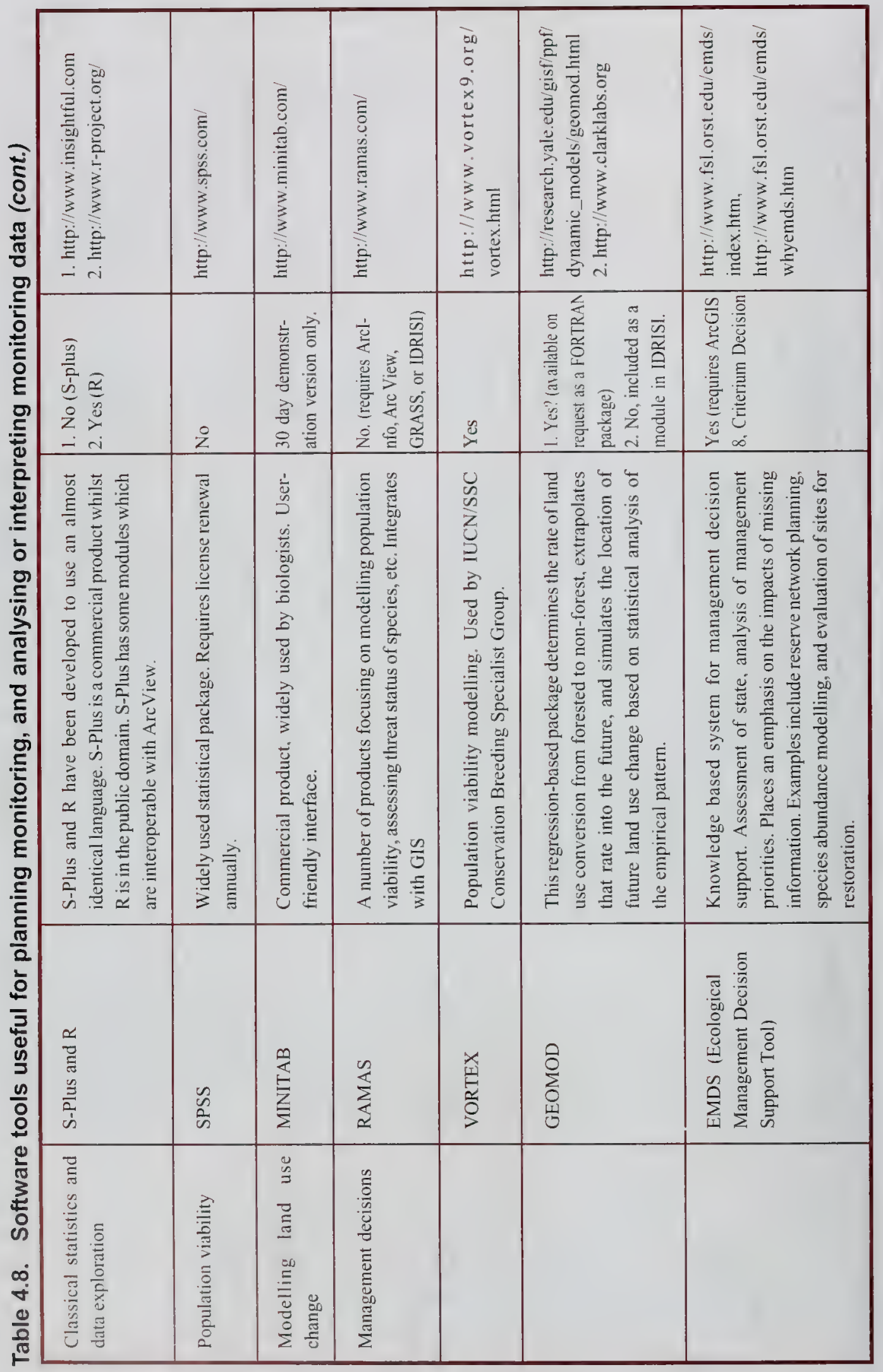


Classical statistics use parametric, non-parametric and exact tests to identify the probability that a null hypothesis is correct (Table 4.7). This formal analysis of a question attaches a level of significance ( $p$-value) to the result. For example, a paired t-test may be used to test the null hypothesis that there is no difference between samples taken from the same permanent plot in different years. If there is a $95 \%$ probability that the null hypothesis is rejected, we say that it is rejected at the $95 \%$ significance level and accept the alternative hypothesis, that there has been a change through time.

Parametric tests require normality in the datasets tested - that is, when value on the $\mathrm{x}$ axis is plotted against the number of data points on the $y$ axis, the results should be a symmetrical bell-shaped curve about the mean value. A two-tailed Kolmogorov-Smirnov test can be used to assess the null hypothesis that the observed distribution function cannot be distinguished from normal. The t-test ANOVA also assumes that each category comes from an underlying symmetric distribution within groups of equal variance (a measure of spread), which can be tested using Levene statistics.

Non-parametric alternatives with exact or Monte Carlo significance can be substituted if these assumptions are not met. These tests make no assumptions about the population distribution. An exact test takes the groups being compared and repeatedly shuffles the values to calculate the test statistic for every possible combination of the observed numbers. The test statistic for the dataset, $a$, is calculated as usual. The exact p-value is then the frequency at which $a$ occurs within the collection of statistics. That is, if it occurs less than $5 \%$ of the time, the null hypothesis is rejected at the $95 \%$ level. Due to computational limitations the suggested maximum number of cases for exact tests is 30 . When there are more cases than this a Monte Carlo estimate of the exact significance level can be used. This method uses repeated random generation of values within the observed margins to calculate probabilities, rather than investigating every combination of the observed values like the exact test.

Many of these tests can be carried out using software that is available via the internet. Table 4.8 therefore lists some statistical resources that exist in the public domain, and offers pointers to resources for other types of analysis, which may be helpful in planning other monitoring or interpreting results. These range from simple tools for single analyses through to management decision-making aids to help you make wider use of monitoring results. They vary considerably in the level of expertise required for their use.

Further guidance on statistical analysis can be found in the references listed in Box 4.4. In addition the 'Statistical/Modeling Tools for Design and Analysis of Conservation Data' and the 'Biodiversity Analysis Package' listed in Table 4.8 are worth a look for their clear explanations of techniques and issues as well as their tools. If an internet site is not accessible, the Wayback Engine at http://www.archive.org can be used to access archived versions of most of these webpages. 


\section{Box 4.4. Recommended sources of further information on statistical analysis.}

\section{Ecological statistics:}

Krebs, C.J. (1998). Ecological Methodology. $2^{\text {nd }}$ edition. Longman. Great help from experimental design through to analysis.

Legendre, P., Fortin. M-J. (1989). Spatial pattern and ecological analysis. Vegetatio 80: 107138. Classic on pattern exploration and spatial autocorrelation.

Digby, P.G.N. \& Kempton, R.A. (1987). Multivariate analysis of ecological communities. Chapman \& Hall, London, UK. Helpful chapter on data exploration.

Wilkinson, L. (1992). Graphical displays. Statistical Methods in Medical Research 1: 3-25. On presenting information.

Hurlbert, S.H. (1984). Pseudoreplication and the design of ecological field experiments. Ecological Monographs 54(2): 187-211. Argues for replication of plots to avoid this issue.

Statsoft (2005). Electronic Statistics Textbook. http://www.statsoft.com/textbook/stathome.html. Includes guide to time series analysis.

\section{Statistical packages:}

PsychNet-UK (n.d.). Software Packages - Statistics. http://www.psychnet-uk.com/ experimental design/software packages.htm. Accessed 14/4/04. - An excellent list of public domain software, put together for behavioural scientists but as useful for ecologists.

Statlib. http://lib.stat.cmu.edu/. [Not available 14/4/04] - A more technical resource

\section{Ordination and regression:}

Palmer, M. (n.d.) Ordination Methods for Ecologists. http://www.okstate.edu/artsci/botany/ ordinate/. Accessed 13/4/04.

\section{Ecological software:}

Illinois Natural History Society (2004). Clearing house for Ecology Software. http:// nhsbig.inhs.uiuc.edu/. Accessed 14/4/04. - A few programs for wildlife ecology \& statistics

\section{Species distributions:}

Anon (2004). Habitat modelling algorithms. http://www.conserveonline.org/2004/03/a/en/ habitat_modeling_algorithms.doc Accessed 14/4/04.

Buckland, S.T. \& Elston, D.A. (1993). Empirical models for the spatial distribution of wildlife. Journal of Applied Ecology 30: 478-495.

Fielding, J.H., Bell, A.F. (1997). A review of methods for the assessment of prediction errors in conservation presence/absence models. Environmental Conservation 24(1): 38-49.

Pearce. J. \& Ferrier, S. (2000). Evaluating the predictive performance of habitat models developed using logistic regression. Ecological Modelling 133(3): 225 - 245. - using the ROC curve for model thresholds. 


\subsubsection{The use of Geographical Information Systems (GIS) in analysis of monitoring data}

The first role of GIS is in data visualisation and exploration. The ability to overlay datasets gives GIS a unique role in map design and exploratory analysis. Field data can be brought together with remote sensing data or existing digitised maps. In a monitoring situation, maps of species or habitat distributions may be compared to assess change through time, and the area and locations of change calculated.

More advanced analytical functions are also available to an expert user, or GIS datasets may be exported for analysis in statistical software. Most GIS packages will allow summary statistics to be calculated, will perform correlations between maps of different variables, and may be programmed to carry out a range of modelling activities limited only by available data, computational capacity and technical skill.

\section{Species distributions}

Species surveys based on a stratified or random sample do not by themselves create a comprehensive distribution map. However, such field data may be interpolated using GIS to fill in the gaps. A map of the probable species distribution can be created, by analysing species observations together with maps of the key factors known to influence their distribution. These may include climate, snow cover, altitude, soil type and vegetation cover. A number of different techniques of varying complexity are employed by the tools listed in Table 4.7. Some are fully integrated with a GIS, whilst others require the relevant data to be extracted prior to analysis. One commonly-used technique is logistic regression, which requires records of species presence and absence.

A set of such species maps can be compiled to allow the identification of areas likely to have a high species richness. This exercise can be carried out for endemic or threatened species to highlight areas of particular conservation interest. These estimates can be "groundtruthed' by field surveys.

\section{Ecological indicators}

As well as helping to interpret field data, GIS procedures can be used to derive new variables from the vegetation datasets. Landscape ecology metrics can themselves serve as indicators for protected area management objectives. For example, a conservation objective may be to limit forest fragmentation, which can then be monitored for the reserve as a whole using landscape metrics. Measures of different aspects of fragmentation, such as local forest density, patch area and distance to core areas of forest can be monitored alone or combined (Kapos et al. 2000).

\section{Scenarios and management planning}

When a species distribution has been successfully modelled and mapped onto the current environment of the reserve, it is possible to use the same model to evaluate the potential long-term impacts of environmental change. For example, if a management plan involves altering an area's habitat type, the model can indicate which species one would expect to lose from the area, and which might be expected to eventually colonise the new habitat patch. These techniques have been frequently used to simulate the impacts of climate and land use change. 
For a more sophisticated analysis, population viability models can be applied within the estimated range of the species, encompassing issues such as minimum habitat requirements and population dynamics (Lindenmayer et al. 1995). This requires much more information about the species in question, but in return provides an estimate of whether the species has a long term future in the areas currently occupied.

\subsection{Report results to stakeholders}

The results of monitoring need to be communicated to several different categories of stakeholders, each of which will have different interests and abilities to interpret and use the results. The manager of the biodiversity monitoring programme needs to identify the stakeholders or users of the monitoring results and assess both their decision-making needs and the most appropriate forms for communication of the information. For example, the manager of the protected area will require written reports and maps, with an analysis of the extent of progress in reaching the conservation objectives. The report should have a summary and may include recommendations for management actions, based on the interpretation of the results. Another key stakeholder group is local communities and their leaders within or near to the protected area. The most appropriate means to present the monitoring results to these groups may be in a much more graphical format, in the local language, and without technical terms. Other important stakeholder groups include government agencies and statutory bodies concerned with the protected area, donors and supporter groups, tourists, and the news media. Whilst monitoring is a repeated activity the timing of the reporting of results should be designed to meet the decision-making needs of the stakeholders.

When reporting monitoring results it is important to ensure credibility in the information. This may be obtained by requesting a review of the results by other experienced and technically-competent colleagues. Similarly, it is important to have documentation of the methodologies and field results in an accessible form, such as the monitoring protocols and database and GIS information management documentation. Communication of the monitoring results should also be considered a two-way process, with the managers of the protected area and its monitoring programme listening to feedback on both the interpretation of the results and the ways in which they are presented.

\subsection{Review the monitoring programme}

It will normally take a couple of years to establish a monitoring programme. And even once it is established, it might be necessary to further develop the programme, as original ideas may not work out as expected or new questions may arise.

During all stages, it is important to continuously review the development of the monitoring programme, by comparing it with its original or adapted objectives and plans. Such a review is important to be able to take action when things are not developing as planned. The review is also important in relation to the quality assurance of the scheme and its outcomes. The review will show whether a scheme has been implemented according to the agreed work plan and protocols, and hence whether scientifically valid results can be derived from it. 
Issues that should be considered within the review include:

- was the intended number of sample plots achieved or is the number increasing according to schedule?

a was the intended frequency of visits to the plots achieved?

- was the intended sampling strategy well applied?

- did the planned field methods work?

a were personnel sufficiently trained?

- have methods been applied consistently?

- were the data and analysis adequate to reliably establish if each objective was achieved?

- were there unforeseen events that affected the monitoring or the achievement of the objectives?

were the monitoring resources adequate, and are more required?

If the review shows that there are some problems in the development of the programme, then constraints should be identified and solutions sought. However, in some cases the original plans may turn out to be impractical and may therefore have to be modified.

But one should be aware of the impacts of changing the monitoring programme, as it might severely influence the possibilities for analysing long-term trends. Every change of the program e.g. in methods or sampling strategy, should be clearly registered and it's consequences should be analysed beforehand. 


\section{References}

Abbot, J. and Guijt, I. 1998. Changing views on change: participatory approaches to monitoring the environment. International Institute for Environment and Development, London, UK.

Bibby, C.J., Burgess, N.D. \& Hill, D.A. 1992. Bird Census Techniques. Academic Press, London.

Bliss J., Aplet G., Hartzell C., Harwood P., Jahnige P., Kittredge D., Lewandowski S., Soscia M. L., Gray G. J. e., Enzer M. J. e. and Kusel J. 2001. Community-based ecosystem monitoring. Journal of Sustainable Forestry 12: 143-167.

Bliss J., Aplet G., Hartzell C., Harwood P., Jahnige P., Kittredge D., Lewandowski S., Soscia M. L., Gray G. J. e., Enzer M. J. e. and Kusel J. 2001. Community-based ecosystem monitoring. Journal of Sustainable-Forestry 12: 143-167.

Bonham, C.D. 1989. Measurements for Terrestrial Vegetation. John Wiley, New York.

Borrini-Feyerabend, G., M. T. Taghi Farvar, J. C. Nguinguiri, and V. A. Ndangang. 2000. Co-management of natural resources: organising, negotiating and learning-by-doing. GTZ and IUCN, Kasperak Verlag, Heidelberg, Germany.

Burrough, P. A. and McDonnell, R. (1998) Principles of Geographical Information Systems. Oxford University Press.

Buckland, S.T., Anderson, D.R., Burnham, K.P., Laake, J.L ., Borchers, D.L. \& Thomas L. 2001. Introduction to distance sampling: estimating abundance of biological populations. Oxford University Press, Oxford.

Callicott J. B., Crowder L. B. and Mumford K. 1999. Current normative concepts in conservation. Conservation Biology 13: 22-35.

CCW. 1996. A guide to the production of management plans for nature reserves and protected areas. Countryside Council for Wales, Bangor.

Cochran, W.G. 1977. Sampling Techniques (3rd edn). John Wiley \& Sons, Chichester.

Daily, C. G., editor. 1997. Nature's services: societal dependence on natural ecosystems. Island Press, Washington, D.C. 
Danielsen, F., D. S. Balete, M. K. Poulsen, M. Enghoff, C. Nozawa, and A. E. Jensen. 2000. A simple system for monitoring biodiversity in developing countries. Biodiversity and Conservation 9:1671 1705.

Danielsen, F., M. M. Mendoza, P. Alviola, D. S. Balete, M. Enghoff, M. K. Poulsen, and A. E. Jensen. 2003. Biodiversity monitoring in developing countries: what are we trying to acheive? Oryx 37: 407-409.

Elzinga, C. L., D. W. Salzer, J. W. Willoughby, and J. P. Gibbs. 2001. Monitoring plant and animal populations. Blackwell Scientific Publications, Abingdon, UK.

Fabbro, L. 2000. Amazonia Biodiversity Estimation using Remote Sensing and Indigenous Taxonomy. Associação Amazonia. Project presented at the European Space Agency Symposium 2000, Gotheborg, Sweden, 16 - 21 October 2000. http://www.amazonia.org/ SustainableDevelopment/Jauaperi/Biodiversity/ALMA/ABDE/index.htm. Accessed 14/4/04.

Feinsinger, P. 2001. Designing field studies for biodiversity conservation. 212 pp. Island Press, Washington D.C.

Fuller J. 1998. Participatory Monitoring of forest resources: Current methodologies being developed in Thailand. Rural Development Forestry Network Paper 23e. ODI, London.

Goldsmith, B., editor. 1991. Monitoring for conservation and ecology. Chapman and Hall, London.

Hill, D.A., Fasham, M., Tucker, G., Shewry, M. \& Shaw, P. (Eds.) in press). The handhook of biodiversity methods: Survey, evaluation and monitoring. Cambridge University Press.

Hirons, G., B. Goldsmith, and G. Thomas. 1995. Site management planning. in W. J. Sutherland and D. A. Hill, editors. Managing habitats for conservation. Cambridge University Press, Cambridge.

Hockings, M., S. Stolton, N. Dudley, and J. Parrish. 2001. The enhancing our heritage' toolkit - Book 2. The workbook on how to build assessment, monitoring and reporting systems on the management effectiveness of World Heritage Sites. UNESCO / IUCN.

Inskipp, C. 1989. A popular guide to the birds and mammals of the Annapuma Conservation Area. ACAP, Kathmandu. 54pp.

IUCN 2004. 2004 IUCN Red List of Threatened Species. <www.redlist.org>.

Jackson, R. 1990. Threatened wildlife, crop and livestock depredation and grazing in the Makalu, working paper publication series. Report $12.75 \mathrm{pp}$ 
Jackson, R. M., G. Ahlbom. M. Gurung, and S. Ale. 1996. Reducing livestock depredation losses in the Nepalese Himalaya. R. M. Timm and A. C. Crabb (eds.). Proceedings of the 17th Vertebrate Pest Conference. University of California, Davis, pp 241-47.

Jackson, R. Richard, C. and Gurung, P. 2001. Enhancing participatory assessment capacity in range and management and livestock wildlife interaction

Jackson, R. Ahlborn, G. Ale, S. Gurung, D. Gurung, M. and Yadav, U.R. 1994. Reducing livestock depredation losses in the Nepalese Himalaya: A case study ACA B

Kapos, V., Lysenko, I. \& Lesslie, R.G. 2000. Assessing forest integrity and naturalness in relation to biodiversity. FAO/UNEP-WCMC, Cambridge, UK.

KMTNC 1994. Biodiversity Conservation Data Project, Final Report. King Mahendra Trust for Nature Conservation, Kathmandu, Nepal.

KMTNC 1997. Annapurna Conservation Area Management Plan. King Mahendra Trust for Nature Conservation, Kathmandu, Nepal.

Krebs, C. J. 1999. Ecological methodology, 2nd ed. 620 pp. Benjamin/Cummings, Menlo Park, California.

Kremen C., Merenlender A. M. and Murphy D. D. 1994. Ecological monitoring: a vital need for integrated conservation and development programs in the tropics. Conservation Biology 8: 388-397.

Lawrence A. 2002. Participatory assessment, monitoring and evaluation of biodiversity: Summary of the ETFRN internet discussion 7-25 January 2002. Website: http:// www.etfrn.org/etfrn/workshop/biodiversity/index.html.

Lawrence A., Malla Y. B., Paudel K. C. and Barnes R. 2003. Why biodiversity? A holistic approach to participatory monitoring of community forestry in Nepal. In Proceedings of International conference on Himalayan Biodiversity. Kathmandu, Nepal.

Linden, H., Helle, E., Helle, P. and Wikman, M. 1996. Wildlife triangle scheme in Finland: methods and aims for monitoring wildlife populations. Finnish Game Research 49 4-11.

Lindenmayer, D.B., Burgman, M.A., Ak•akaya, H.R., Lacy, R.C. \& Possingham, H.P. 1995. A review of the generic computer-programs ALEX, RAMAS/space and VORTEX for modelling the viability of wildlife metapopulations. Ecological Modelling 82: 161-174.

Margules, C., and M. B. Usher. 1981. Criteria used in assessing wildlife conservation potential: a review. Biological Conservation 21:79-109.

NASA 2003. Remote Sensing Tutorial. Vegetation Applications: Agriculture, Forestry, And Ecology. Available from: http://rst.gsfc.nasa.gov/Sect3/Sect3 1.html. Accessed 7-Oct-2003. 
Nature Conservancy Counci1. 1989. Guidelines for selection of biological SSSIS, 1998 edition. Nature Conservancy Council, Peterborough.

Oaks, J.L., Gilbert, M., Virani, M.Z., Watson, R.T., Meteyer, C.U., Rideout, B.A., Shivaprasad, H.L., Ahmed, S., Chaudhry, M.J.I., Arshad, M., Mahmood, S., Ali, A. and Khan, A. A. 2004. Diclofenac residues as the cause of vulture population decline in Pakistan. Nature 427: 630-633.

Longley, P. A., Goodcjild, M. F., Maguire, D. J. and Rhind, D. W. (2001) Geographical Information Systems and Science, John Wiley and Sons

Obura D. O. 2001. Participatory monitoring of shallow tropical marine fisheries by artisanal fishers in Diani, Kenya. Bulletin of Marine Science 69: 777-791.

Oli, M.K, 1991. The Ecology of Conservation of the Snow Leopard ( Panthera uncia) in the Annapurna Conservation Area, Nepal

Pimbert M. P. and Pretty J. N. 1995. Parks, people and professionals: putting 'participation' into protected area management. Discussion Paper 57 UNRISD / IIED / WWF.

Posey, D. A., editor. 2000. Cultural and spiritual values of biodiversitn: A complementary. contribution to the global biodiversity assessment. UNEP.

Pretty J. N. 1994. Alternative systems of inquiry for sustainable agriculture. IDS Bulletin 25: 37-48.

Rabinowitz, D. 1981. Seven forms of rarity. Pages 205-217 in H. Synge, editor. The biological aspects of rare plant conservation. Wiley, Chichester.

Ramsar Bureau. 2002. New guidelines for management pianning for Ranisar sires and other wetlands. Ramsar Bureau, Gland, Switzerland.

Ratcliffe, D. A. 1977. A nature conservation review: The selection of biological sites of national importance to nature conservation in Britain. Cambridge University Press, Cambridge.

Salafsky, N., and R. Margoluis. 1999. Threat reduction assessment: a practical and costeffective approach to evaluating conservation and development projects. Conservation Biology 13:830-841.

Salim A., Brocklesby M. A., Tiani A. M., Tchikangwa B., Sardjono M. A., Porro R., Woefel J. and Pierce Colfer C. J. 2001. In search of a conservation ethic. In: Pierce Colfer C.J. and Byron Y. (Ed) People managing forests: The links betwe'n human well-being and sustainability. pp. 155-170. Resources for the Future, Washington. 
Sheil, D., Puri, R.K., Basuki, I., van Heist, M., Syaefuddin, Rukmiyati, Sardjono, M.A., Samsoedin, I., Sidiyasa, K., Chrisandini, Permana, E., Angi, E.M., Gatzweiler, F., Johnson, B. and Wijaya, A. 2002. Exploring biological diversity, environment and local people's perspectives in forest landscapes. Centre for International Forestry Research, Bogor, Indonesia.

Shultz, S., Baral, H.S., Charman, S., Cunningham, A.A., Das, D., Ghalsasi, G.R., Goudar, M.S., Green, R.E., Jones, A., Nighot, P., Pain, D.J. and Prakash, V. 2004. Diclofenac poisoning is widespread in declining vulture populations across the Indian subcontinent. Proceedings of the Royal Society London B (supplement). DOI 10.1098/rsbl.2004.0223 http://www.vulturedeclines.org/gypdiclol.pdf

Smith, P. G. R., and J. B. Theberge. 1986. A review of criteria for evaluating natural areas. Environmental Management 10:715-734.

Southwood, T. R. E. 1978. Ecological methods, 2nd edition. Chapman \& Hall, London.

Spellerberg, I. F. 1991. Monitoring ecological change. Cambridge University Press, Cambridge.

Spellerberg, I. F. 1992. Elaluation and assessment for conservation. Chapman \& Hall, London.

Steinmetz R. 2000. Ecological survers, monitoring and the involvement of local people in protected areas of Lao P.D.R. Evaluating Eden Series, Discussion Paper 13. WWF Thailand Programme Office, Bangkok, Thailand.

Stockdale M. C. and Ambrose B. 1996. Mapping and NTFP inventory: participatory assessment methods for forest-dwelling communities in East Kalimantan, Indonesia. In: Carter J. (Ed) Recent approaches to participatory forest resource assessment, pp. 170211. Overseas Development Institute, London.

Sutherland, W. J., editor. 1996. Ecological census techniques. Cambridge University Press, Cambridge.

Thapa, K. 2000. Livestock depredation in Nar and Phu valley, Manang region of Annapurna Conservation Area, Nepal, King Mahendra Trust for Nature Conservation/Annapurna Conservation Area Project

Thomas, L. \& Krebs, J.K. 1997. A review of statistical power analysis software. Bulletin of the Ecological Society of America 78, 128-139.

Thomas, L., and J. Middleton. 2003. Guidelines for management of protected areas. IUCN, Gland, Switzerland and Cambridge, UK.

Usher, M. B., editor. 1986. Wildlife conservation evaluation. Chapman \& Hall, London.

Van Rijsoort J. and Zhang J. F. 2002. Developing of participatory resources monitoring in two Nature Resenes in Yuman, P.R. China. Website: http:/www.etfrn.org/etfrn/workshop/ biodiversity/index.html. 


\section{Acronyms}

ACA Annapurna Conservation Area

ACAP

Annapurna Conservation Area Project

CAMC

Conservation Area Management Committee

CE

Conservation Education

GIS

Geographical information system

GPS

Global positioning system

KMTNC

King Mahendra Trust for Nature Conservation

VDC

Village Development Committee 


\section{Glossary}

Accuracy

Attributes

Feature

Key area

Macroplot

Monitoring

Observation

Population

Precision

Primary plot and secondary plots
The closeness of an estimated value to the true value.

Characteristics, qualities or properties of a feature that are inherent to, and inseparable from, the feature (CCW, 1996). For species these may include population size, structure, habitat requirements, distribution and other parameters. Attributes of habitats may include key species, composition, structure, supporting processes and other parameters.

A habitat, habitat matrix, species or a species assemblage occurring on a site.

An area that is subjectively selected for sampling within because it is assumed to be important or representative of a larger area. Statistical inferences can only be made for the key area.

Relatively large regular shaped areas laid over the bulk of the target area, within which sampling units such as quadrats, lines or points are located. They facilitate the positioning of sampling units.

"The collection and analysis of repeated observations or measurements to evaluate changes in condition and progress toward meeting a management objective" (Elzinga et al. 2001).

A record (e.g. measurement of height, count of numbers) taken from a sample unit.

Any collection of individual items or units which are the subject of investigation. The population is the total number of units, from which we usually take subsets or samples.

The closeness of the sample measurements to each other. An estimate is more precise if it has a smaller standard deviation.

Plots in two-stage (or multi-stage sampling), where further sampling (i.e. with secondary plots) is carried out within the primary plots. 
Sample

Sample unit, Sampling unit

Sample population The population or area over which samples may be drawn from.

A subset of the units in a population which represents the population as a whole. If a sample is to be truly representative, the sample must be drawn randomly (i.e. free from bias) from the population.

A sample unit is an individual population unit from a sample. A sampling unit is a collection of observations with specified dimensions (e.g. a quadrat). A set of these comprises a sample.

Statistical population The entire set of observations across all the samples, from which statistical inferences are made.

Surveillance

An extended programme of surveys systematically undertaken to provide a series of observations to ascertain the variability that might be encountered over time (but without preconceptions of what these might be).

Target population The population that we are interested in (e.g. the population of a species, or an area of habitat, that we are managing and have set a conservation objective for). 


\section{Example protocols from the ACAP biodiversity programme}

\subsection{Introduction}

These monittoring protocols and data collection sheets have been developed by KMTNC with the assistance of UNEP-WCMC over the period 2003 to 2005. They are presented as examples of the types of conservation objectives for which protocols can be developed and the information and procedures required for monitoring

\subsection{ACAP Monitoring protocol for Snow Leopard (Uncia uncia)}

\section{Monitoring objectives}

\section{Reasons for monitoring:}

- Snow Leopard is an Endangered Species and is legally protected by the National Parks and Wildlife Conservation (NPWC) Act 2029 of HMG/N, listed in Appendix I of Convention on International Trade in Endangered Species of Wild Fauna and Flora (CITES) and as Endangered in IUCN Red Data Book.

- It is an indicator species and one of the top level predator of alpine ecosystem within ACA

- Population in decline in last few years due to unknown reasons (possibly persecution and poaching)

d Flagship species (considerable international interest)

\section{Conservation objectives for the resource:}

To maintain the frequency of occurrence and distributional range of snow leopard within ACA as indicated by baseline presence level (to be determined in 2004-2006).

\section{Monitoring population I area and sub-units:}

The target monitoring area is all suitable habitat in the ACA. But, due to the large size of the ACA $\left(7,629 \mathrm{~km}^{2}\right)$, the steep terrain and numerous cliffs it is not considered feasible to undertake monitoring of all suitable habitats. Monitoring fieldwork will therefore focus on the following key populations in Sangta, Bhena (Mustang), the Nar and Pho valleys, and Khangsar (Manang). [Sites to be confirmed and key areas mapped]

Sample areas within each key area will be land that is below $5,500 \mathrm{~m}$, and excluding areas of permanent snow or ice and ground that is too steep to safely access.

Presence in other areas to be monitored by reporting of incidental sightings to wildlife recording scheme. 


\section{Frequency:}

There has been some evidence of declines in this globally threatened species. Therefore establishment of a monitoring programme and assessment of population trends is a high priority for ACAP. However, the potential range of the species is very large within the ACA and survey work is difficult and time-consuming. It is therefore suggested that monitoring is carried out on a five-year cycle.

All incidental sightings to be reported to the wildlife reporting scheme.

\section{Monitoring Methods}

These methods are largely based on the recommended Snow Leopard Information Management System (SLIMS) survey methods described in the Snow Leopard Manual (Bajimaya 2001). These have been adapted to increase their statisticai applicability and to take into account the difficult terrain and resource limitations on ACAP staff.

\section{Observation types:}

Direct observation of animals (although very unlikely), calls; and signs (scrapes, scent spraying, faeces, tracks (pugmarks, spoors), prey kills and snow leopard remains.

Pugmark size (to distinguish individuals).

\section{Data type:}

Presence / absence in sample squares and mapped positions of sightings / signs. Counts of signs per transect (see attached recording form Part B).

Possible minimum number of individuals present if pugmarks are detected and measured.

\section{Complete census or sample survey:}

Sample survey.

\section{Sample method:}

Snow leopards are difficult to detect and wide ranging species, with home ranges of 12 $39 \mathrm{sq} \mathrm{km}$ in prime habitat (Bajimaya 2001). Multi-stage sampling will therefore be used to ensure high interspersion of primary samples and more intensive sampling in secondary samples.

Primary sample units will be $3 \mathrm{~km} \times 3 \mathrm{~km}$ sample survey squares. A relatively large primary sample area is used to allow for the likelihood that some of the area will be inaccessible. Secondary samples are $5 \mathrm{~m}$ wide transects within each primary sample.

\section{Sample area / time period:}

The time taken to carry out surveys will vary according to terrain etc. However, the actual time taken searching transects on the 1st survey must be recorded and the same time taken on all repeat surveys. 


\section{Timing of observations:}

Surveys will be conducted in February, March or April, and will start at sunrise. Return surveys should be conducted within the same month of the year as the previous survey.

\section{Potential causes of bias and rules for standardization:}

Snow Leopards are mostly active during dawn and dusk or at night in disturbed areas. There may therefore be a bias against sightings in areas near human habitation or if daily survey periods vary. However, Snow Leopards are extremely difficult to observe directly, and therefore most data are expected to consist of indirect signs of presence. These potential biases are therefore unlikely to be significant in practice. Counts should however start early in the day to maximize potential chances of sightings and because Blue Sheep counts will be conducted at the same time, and these are best carried out near dusk or dawn.

Underestimates may occur where livestock densities are high as trampling tends to destroy Snow Leopard tracks and scrapes etc. Transects should avoid tracks used intensively by livestock.

The effort used to detect signs must be consistent between years. All surveyors must be trained in the recognition of Snow Leopard tracks and other signs. Observers must therefore be very diligent when surveying each transect within the sample squares. Surveys should be carried out by 2 surveyors. If more are present, no more than 2 should be actively searching at any one time.

Care should be taken in extrapolating results where a high percentage of the survey area or primary sample was inaccessible.

\section{Sampling methods}

\section{Temporary or permanent sample location:}

Permanent primary and secondary samples.

\section{Method for sample location:}

Samples should be located randomly within each key monitoring area, but due to the wide ranging nature of the species, good interspersion and independence of samples is required. Primary samples will therefore be located by restricted random sampling.

At least $50 \%$ of each randomly located survey square must fall within the sample area and accessible areas. Primary squares must also be $>1 \mathrm{~km}$ apart.

Transects within survey squares will be placed by judgement along routes that are considered likely to be used by Snow Leopards, e.g. along ridgelines, cliff bases and river bluffs, according to guidance given in the Snow Leopard Manual. A sufficient number of transects should be selected to take up $6-8$ hours of searching (not including travel time between). Transects should be subdivided according to appropriate land features (e.g. extent of cliff edge) and the amount of time take to survey each one recorded to aid consistency between subsequent resurveys. Each survey square should contain at 
least $1 \mathrm{~km}$ of defined transects, and transects should aim to be $100-500 \mathrm{~m}$ long, but may be longer if they match obvious continuous topographical features.

Transects should not cross the 5,500 m contour, permanent snow or ice or dangerously steep ground.

The location of transects must be accurately mapped and frequent waymarker positions noted accurately by GPS and photographed.

Note: As transects are located deliberately (by judgement) in areas of good habitat and where signs are likely to be found they produce biased estimates of Snow Leopard abundance. These results can therefore only be used as a rough guide to abundance and should not be compared statistically. However, subsequent changes in relative abundance on transects and the presence of snow leopards in each sample square may be tested if the same transects and methods are used to search these in each subsequent year (see analysis section below).

\section{Number of samples:}

Four squares in Khansgar key area, six in Nar and Pho valleys. [Numbers to be decided for other key areas]

\section{Monitoring Requirements}

\section{Personnel responsible and time required:}

Monitoring to be coordinated by ACAP Monitoring Manager, with sample locations identified by GIS team. Surveys must be carried out by specifically trained ACAP Field Staff.

\section{Experience training necessary:}

Training must be given to all staff undertaking the surveys.

\section{Licence and access permission requirements:}

Not applicable

\section{Equipment required:}

GPS, map of each primary sample square (preferably at $1: 10,000$ or 1:20,000 scale) with marked transects, photographs of key landmarks to aid relocation. Sections on Snow Leopard signs from Snow Leopard Manual (Bajimaya 2001). Standard recording forms must be used, with copies of codes sheets from Snow Leopard Manual. Binoculars, camera, small tape measure (for measuring tracks and scrapes etc). Field safety kit.

\section{Data storage}

Original Data Recording Forms and maps should be safely stored within each Ficld Office and the data entered onto the standard Excel summary form and sent to the Monitoring Manager. 
Field monitoring reports must be submitted with the data. These should document the detailed methods used, including any deviations from this protocol. The reports should include: large scale maps indicating boundaries of the primary sample square (with coordinates for each corner). Transect locations with start and end coordinates; photographs of transect start-points and other important features on the transect (e.g. boulders used as scent marking sites); notes describing each transect (e.g. ridge, streamside), the order in which each was examined and the time taken to examine each transect. The reports should include the original data forms (or cross-checked typed copies) and the SLIMS Codes.

\section{Data analysis}

The main analysis will be of the proportion of sample squares that have signs of Snow Leopard. As sample locations are permanent, changes in the proportion of squares with Snow Leopard may be tested using Mc-Nemar's test (See Elzinga et al. 2001), but sample sizes are unlikely to be sufficient to allow formal statistical significance testing. Any decline in presence should be considered to be of concern and should trigger further investigations (e.g. further more intensive surveys) and appropriate management measures.

A general indication of the relative abundance of Snow Leopards may also be gained from the number of signs recorded per $\mathrm{km}$ of transect (see Snow Leopard Manual for details). Changes in this relative abundance measure may be tested by a paired t-test.

Longer term trends may be examined by regression analysis.

\section{Reporting procedures:}

Every five-years on completion of survey cycle.

\section{Reference}

Bajimaya, S. 2001. Snow Leopard manual. Field techniques for the kingdom of Nepal. WWF Nepal, Kathmandu.

Elzinga, C. L., D. W. Salzer, J. W. Willoughby, and J. P. Gibbs 2001. Monitoring plant and animal populations. Blackwell Scientific Publications, Abingdon, UK. 


\subsubsection{ACAP Snow Leopard data recording form}

\section{Part a: count details}

Date (day/month/year):

\section{Counter details}

Name of lead counter:

Address:

Other observers / trainees present:

\section{Primary sample square}

ACAP Unit Conservation Area:

Nearest town / village:

Survey square number:

Coordinates (plot center):

\section{Visit details}

Start time: End time:
Altitude - $\min (\mathrm{m})$ :

Altitude - max:

Aspect:

Weather conditions:

Temperature (circle): Cold $\left(\left(<5^{\circ} \mathrm{C}\right)\right) / \mathrm{Cool}\left(5-10^{\circ} \mathrm{C}\right) / \mathrm{Mild}\left(10-15^{\circ} \mathrm{C}\right) /$ Warn $\left(15-20^{\circ} \mathrm{C}\right) / \operatorname{Hot}\left(>20^{\circ} \mathrm{C}\right)$

Cloud cover (to nearest $10 \%$ or give range):

Wind direction: Wind speed: still / light / breeze / strong breeze / near gale / gale 
Part b: Observations (use 1 form for each transect) Page ..... of ......

\begin{tabular}{|l|l|l|l|l|}
\hline $\begin{array}{c}\text { Comments } \\
\text { Lead observer }\end{array}$ & Date & $\begin{array}{c}\text { Survey } \\
\text { square }\end{array}$ & Nearset village & Transect no. \\
\hline & & & \\
\hline \multicolumn{2}{|l|}{} & End coordinates & Length $(m)$ \\
\hline Start coordinates & End time & \\
\hline \multicolumn{2}{|l|}{ Start time } & & Duration \\
\hline
\end{tabular}

\section{See notes below and use Codes for SLIMMS Form 2}

\begin{tabular}{|c|c|c|c|c|c|c|c|c|}
\hline 1 & 2 & 3 & 4 & 5 & 6 & 7 & 8 & 9 \\
\hline Site No. & Obs No & Coord & Spp & Alt (m) & Aspect & Slope & Range use & Habitat \\
\hline & & & & & & & & \\
\hline & & & & & & & & \\
\hline & & & & & & & & \\
\hline & & & & & & & & \\
\hline & & & & & & & & \\
\hline & & & & & & & & \\
\hline & & & & & & & & \\
\hline & & & & & & & & \\
\hline & & & & & & & & \\
\hline & & & & & & & & \\
\hline
\end{tabular}

\begin{tabular}{|c|c|c|c|c|c|c|c|c|c|}
\hline 1 & 2 & 10 & 11 & 12 & 13 & 14 & 15 & 16 & 17 \\
\hline Site No. & Obs No & Landform & $\begin{array}{c}\text { Topo } \\
\text { feature }\end{array}$ & $\begin{array}{c}\text { Marked } \\
\text { feature }\end{array}$ & Site type & Sign type & $\begin{array}{c}\text { Sign age } \\
\text { vis }\end{array}$ & $\begin{array}{c}\text { Substrate } \\
\text { type }\end{array}$ & $\begin{array}{c}\text { Sign size } \\
\text { (cm) }\end{array}$ \\
\hline & & & & & & & & \\
\hline & & & & & & & & \\
\hline & & & & & & & & & \\
\hline & & & & & & & & & \\
\hline & & & & & & & & \\
\hline & & & & & & & & \\
\hline & & & & & & & & \\
\hline & & & & & & & & \\
\hline
\end{tabular}

Notes $^{*}$ 1: Number each site where sign observed and number map. 2: Number each observation at each site. 4: Record all large predators (excluding domestic dog).

Comments: (with respect to site \& obs no.) 


\subsection{ACAP Monitoring protocol for Himalayan Griffon (Gyps himalayensis) and other vultures}

\section{Monitoring objectives}

\section{Reasons for monitoring:}

- Population in decline in last few years due to unknown reasons

- Flagship species

\section{Conservation objectives for the resource:}

To determine baseline relative population abundance levels and to maintain these over the next ten years (to 2015) within the Annapurna Conservation Area (ACA).

\section{Monitoring population / area and sub-units:}

The target population is the breeding population with the $\mathrm{ACA}$, covering all Unit Conservation Areas (Lomanthang, Jomson, Ghandruk, Lwang, Sikles, Bhujung, Manang). The sample population will be birds visible from selected lookout points within the target population area that are within accessible areas and which are not above $5000 \mathrm{~m}$, or are not rock or permanently covered in ice or snow.

\section{Frequency:}

There have been major declines in many vulture species and populations with the Indian subcontinent in recent years, including Himalayan populations of White-rumped Vulture (Gyps bengalensis), Cliff Vulture (Gyps indicus) and Slender Billed Vulture (Gyps tenuirostris). There has also been some indications of a decline in Himalayan Vulture populations within the ACA (Baral et al. 2002). Establishment of a monitoring programme and assessment of population trends for Himalayan Vultures and other vulture species is therefore a high priority for ACAP.

Annually for first 5 years and then every 2 years afterwards. Kill counts will be made whenever encountered by ACAP staff.

\section{Monitoring methods for soaring birds}

Due to the large size of the ACA $\left(7,629 \mathrm{~km}^{2}\right)$, the steep terrain and numerous cliffs it is not considered feasible to undertake monitoring of birds at nesting colonies. Most would be inaccessible and monitoring of only larger key colonies could produce substantially biased results.

\section{Observation types:}

Direct observation of soaring vultures (all species) and other large soaring raptors and ravens.

\section{Data type:}

Counts of total bird sightings (i.e. maximum possible number of individuals), estimated number of individuals and minimum number of individuals during a timed count. See attached Soaring Vulture recording Form. 


\section{Complete census or sample survey:}

Sample survey.

\section{Sample method:}

Sample units will be point counts from selected vantage points.

\section{Sample area / time period:}

All visible birds will be counted (i.e. no fixed area) during a standard 4 hour count period

\section{Timing of observations:}

Counts will be made between 10.00 and 14.00, between 1st June and 30th June (i.e. when breeding birds are present and feeding young). [Seasonal timing to be confirmed]

\section{Potential causes of bias and rules for standardization:}

Soaring is dependent on the presence of thermals or updrafts and is therefore greatly affected by weather conditions. Counts should, therefore, only be made during suitable soaring conditions, i.e. in the absence of rain, fog, low cloud or complete cloud cover. Counts should also only be carried out during the middle of the day when solar energy and hence thermal activity is at its greatest, i.e. $10.00-14.00 \mathrm{hrs}$.

It is difficult to avoid double counting of vultures as they often back track during their foraging flights. Care must therefore be taken in estimating the number of individual birds seen. Details of each bird's age and plumage should therefore be noted during sightings and consistent decision rules used to estimate the number of individuals seen. A minimum number of individuals seen will also be calculated on the basis of simultaneous sightings plus any subsequent birds of clearly different age or plumage.

The effort used to detect birds must be consistent between years. Only one counter should detect birds (i.e. if other observers / trainees are present, then they should not indicate birds to the lead counter or include birds seen by them and not the lead counter on the recording forms). Observers should remain vigilant throughout the four hour period and should make regular scans of the sky with their binoculars (e.g. a 3600 scan every 5 minutes). Telescopes should not be used to detect birds that are not normally visible with binoculars.

\section{Sampling methods for soaring birds}

\section{Temporary or permanent sample location:}

Permanent points

\section{Method for sample location:}

Vantage points will be selected by judgment from within randomly placed $5 \times 5 \mathrm{~km}$ squares. Each randomly placed square must, however, be more than $10 \mathrm{~km}$ apart from any previously located squares. If this is not the case then the selected square must be discarded and another square randomly placed. 
Randomly placed squares that overlap with the ACA boundary should be retained if a suitable vantage point is present and counts made of all birds seen whether or not they are over the ACA.

Vantage points should be selected so that they give as wide a view of the surrounding landscape as possible. Within forested areas they should be at or above the treeline or as high as possible. Suitable vantage points will include peaks and ridges. North facing slopes should be avoided if possible, unless they provide good vantage points overlooking nearby south facing slopes. Considerable care should be used in selecting vantage points as these will be permanently used for all subsequent monitoring counts. It is therefore recommended that vantage points are selected by careful reference to maps, supplemented with reconnaissance visits to several potential sites before final selections of vantage points are made.

The location of vantage points should be accurately recorded by GPS, photographed and mapped.

\section{Number of samples:}

One vantage point will be used in each $5 \times 5 \mathrm{~km}$ square and twenty random sample squares will be selected and counted in the 1 st year. The data obtained from the 1st year will then be used to calculate a suitable sample size for subsequent monitoring. If less than twenty squares are acceptable, then excess sample squares will be randomly discarded.

Two counts should be made at each vantage point each year during the survey period.

\section{Use of multi-stage sampling:}

As vultures are very wide raging species, high interspersion of samples is required and therefore multi-stage sampling is not applicable.

\section{Monitoring methods for birds at carcasses}

This method is based on the standard method used by the Vulture Decline Project.

\section{Observation types:}

Direct counts of birds at carcasses. If possible several photographs of birds present at the carcass (and soaring above it) should be taken for verification by an ornithologist of the species present and their age classes.

\section{Data type:}

Counts of vultures according to species and age class. Counts of birds showing signs of neck drooping (i.e. disease). Counts of other scavengers.

\section{Complete census or sample survey:}

Sample survey

\section{Sample method:}

Birds present at carcasses, forms to be competed for all carcasses found. irrespective of whether vultures are present. 


\section{Sample area / time period:}

No restriction, all birds visible at the carcass when discovered.

\section{Timing of observations:}

No restriction

\section{Potential causes of bias and rules for standardization:}

Vulture numbers at carcasses may vary depending on the number of carcasses available (i.e. if carcasses are plentiful then birds may be widely dispersed with low numbers at each carcass). Thus apparent changes in numbers may result from variation in carcass availability. Trends should therefore be compared with the number of carcasses found.

The number of carcasses found will, however, vary according to survey effort including the willingness to look for and record carcasses and the amount of time spent in the field. The importance of looking for and recording all carcasses encountered should therefore be emphasized to all field staff. Results should also be related to the amount of time spent in the field by staff (which is to be recorded as part of the ACAP wildlife recording scheme, see separate protocol).

\section{Sampling methods for birds at carcasses}

\section{Temporary or permanent sample location:}

Temporary

\section{Method for sample location:}

Chance encounters by ACAP staff with carcasses during other field activities.

\section{Number of samples:}

Variable depending on carcass numbers and field effort.

\section{Use of multi-stage sampling:}

Not applicable

\section{Monitoring requirements}

\section{Personnel responsible and time required:}

Monitoring to be coordinated by ACAP Monitoring Manager [to be identified], with sample locations identified by GIS team. Counts of soaring birds to be carried out by specifically trained ACAP Field Staff. Counts of birds at carcasses to be carried out by all ACAP staff who have received basic training in the method and vulture identification.

\section{Experience training necessary:}

Training must be given to all staff undertaking the surveys. However, expertise in identification of all birds is not necessary, and the surveys can be carried out by ACAP personnel or other members of the local communities who are competent in vulture identification. 


\section{Licence and access permission requirements:}

Not applicable

\section{Equipment required:}

Binoculars (consistent magnification between surveys, and preferably $10 \mathrm{x}$ magnification), GPS, map of site location of vantage point and photograph of vantage point to aid relocation. Bird identification guide and tables summarizing vulture identification criteria (see Bombay Natural History Society 2001). Standard recording forms must be used. Telescopes may also be used for verifying identification, age and plumage features of birds detected through binoculars. Field safety kit.

\section{Data storage}

Original Data Recording Forms should be safely stored within each Field Office and the data entered onto the standard Excel summary form and sent to the Monitoring Manager [ACAP to devise based on the recording forms]. Carcass recording forms should also be copied and then sent to the appropriate contact person of the Vultures Decline Project.

\section{Data analysis}

For counts of soaring birds; the number of individuals (maximum number, estimated number of individuals and minimum number of individuals) of each species shall firstly be averaged over the two counts at each vantage point. Year to year changes in relative abundance should then be examined by calculating a mean percentage difference with confidence limits across the set of vantage points.

Longer term trends may be examined by regression analysis.

It is anticipated that the analysis of carcass counts will be made by the Vulture Decline Project

\section{Reporting procedures:}

Annually

\section{$\underline{\text { References }}$}

Bombay Natural History Society (2001). Proceedings of a Gyps spp. Vulture monitoring workshop. Available at http://www.vulturedeclines.org/home.asp

Baral, H.S., Giri, J.B., Choudhary, H. Basnet, S., Watson, R. and Virani, M. (2002). Surveys of Himalayan Griffon Gyps himalayensis in the Nepalese Himalayas. Final report 2002 to The Peregrine Fund, USA. 


\subsubsection{ACAP soaring vulture data recording form}

\section{Part a: count details}

Date (day/month/year):

Count of 2

Counts must be undertaken between $10.00-14.00$. Please note any discrepancies or interruptions in comments section.

\section{Counter details}

Name of lead counter:

Address:

Other observers / trainees present:

\section{Count location}

ACAP Unit Conservation Area:

Sample point code:

Altitude:

\section{Visit details}

\section{Weather conditions at start of count:}

Temperature (circle one): Cold $\left(<5^{\circ} \mathrm{C}\right) \quad \mathrm{Cool}\left(5-10^{\circ} \mathrm{C}\right)$ Hot $\left(>20^{\circ} \mathrm{C}\right)$

Aspect:
Nearest town / village:

Coordinates:

Cloud cover (estimate to nearest $10 \%$ ):

\section{Weather conditions at end of count:}

Temperature (circle one): Cold $\left(<5^{\circ} \mathrm{C}\right) \quad \mathrm{Cool}\left(5-10^{\circ} \mathrm{C}\right)$

Mild $\left(10-15{ }^{\circ} \mathrm{C}\right)$

Hot $\left(>20^{\circ} \mathrm{C}\right)$
Mild $\left(10-15^{\circ} \mathrm{C}\right)$

Warm $\left(15-20^{\circ} \mathrm{C}\right)$

Cloud cover (estimate to nearest $10 \%$ ):

Optical equipment used:

Broad habitat type (circle one main type present within $5 \times 5 \mathrm{~km}$ square: a) Cultivation Area b) Deciduous / mixed forest c) Coniferous forest d) Montane deciduous forest / shrubland e) Grassland f) Barren soil / rock g) Permanent snow and ice

\section{Comments}


Part b: Observations

\section{Lead counter: \\ Date:}

\section{Page ..... of ......}

\begin{tabular}{|c|c|c|c|c|c|c|}
\hline \multirow[t]{2}{*}{ Time } & \multirow[t]{2}{*}{ Species* } & \multicolumn{4}{|c|}{ Number of Vultures } & \multirow{2}{*}{$\begin{array}{c}\text { Notes } \\
\text { (see } \\
\text { below) }\end{array}$} \\
\hline & & Juveniles & Sub-adults & Adults & $\begin{array}{c}\text { Age } \\
\text { Unknown }\end{array}$ & \\
\hline & & & & & & \\
\hline & & & & & & \\
\hline & & & & & & \\
\hline & & & & & & \\
\hline & & & & & & \\
\hline & & & & & & \\
\hline & & & & & & \\
\hline & & & & & & \\
\hline & & & & & & \\
\hline & & & & & & \\
\hline & & & & & & \\
\hline & & & & & & \\
\hline & & & & & & \\
\hline & & & & & & \\
\hline & & & & & & \\
\hline & & & & & & \\
\hline & & & & & & \\
\hline & & & & & & \\
\hline & & & & & & \\
\hline & & & & & & \\
\hline & & & & & & \\
\hline & & & & & & \\
\hline & & & & & & \\
\hline & & & & & & \\
\hline & & & & & & \\
\hline & & & & & & \\
\hline & & & & & & \\
\hline & & & & & & \\
\hline & & & & & & \\
\hline & & & & & & \\
\hline & & & & & & \\
\hline
\end{tabular}

* Record all sightings of vultures, Golden Eagle, other eagles, buzzards and Ravens.

Notes: (see code number in Table) 
Part c: Summary of observations

Lead counter:

Date:

\begin{tabular}{|c|c|c|c|c|c|c|}
\hline \multirow[t]{2}{*}{ Species* } & & \multicolumn{5}{|c|}{ Number of Vultures } \\
\hline & & TOTAL & Juveniles & $\begin{array}{c}\text { Sub- } \\
\text { adults }\end{array}$ & Adults & $\begin{array}{c}\text { Age } \\
\text { Unknown }\end{array}$ \\
\hline \multirow[t]{3}{*}{ Himalayan Griffon } & Max indv & & & & & \\
\hline & Est indv & & & & & \\
\hline & Min indv & & & & & \\
\hline \multirow[t]{3}{*}{ Bearded Vulture } & Max indv & & & & & \\
\hline & Est indv & & & & & \\
\hline & Min indv & & & & & \\
\hline \multicolumn{7}{|l|}{ Other species } \\
\hline & Max indv & & & & & \\
\hline & Est indv & & & & & \\
\hline & Min indv & & & & & \\
\hline & Max indv & & & & & \\
\hline & Est indv & & & & & \\
\hline & Min indv & & & & & \\
\hline & Max indv & & & & & \\
\hline & Est indv & & & & & \\
\hline & Min indv & & & & & \\
\hline & Max indv & & & & & \\
\hline & Est indv & & & & & \\
\hline & Min indv & & & & & \\
\hline & Max indv & & & & & \\
\hline & Est indv & & & & & \\
\hline & Min indv & & & & & \\
\hline & Max indv & & & & & \\
\hline & Est indv & & & & & \\
\hline & Min indv & & & & & \\
\hline & Max indv & & & & & \\
\hline & Est indv & & & & & \\
\hline & Min indv & & & & & \\
\hline
\end{tabular}

\section{Comments:}




\section{Counter details Recording form for scavengers on carcasses}

\author{
Name of lead counter \\ Address \\ State
}

Visit details

Date (day / month / year)

Time of start of count $(\mathrm{HH}: \mathrm{MM})$ : $\mathrm{am} / \mathrm{pm}$ (circle one)

Time of end of count (HH : MM) : am / pm (circle one)

Temperature (circle only one) cold cool mild warm hot

Number of fresh livestock carcasses (circle only one)
Number of counters

Details of carcass dump

Name (if any)

Nearest village

Town and District $a b c c$

e $f \mathrm{~g} \mathrm{~h}$

Broad Habitat Type

$2-5 \quad 6-10 \quad 11-25 \quad>25$

\section{Counts of vultures (include sick birds in the counts of perched birds)}

Total number of Gyps vultures (all species) perched at the carcass

Total number of Gyps vultures (all species) soaring above the carcass

Please fill out the table below for the first 50 that you see when scanning the dump, or all birds if there are less than 50 . If you do not have time to age the birds, put total numbers of each species in 'Age Unknown'

\begin{tabular}{|c|c|c|c|c|c|}
\hline \multirow[b]{2}{*}{ Species } & \multicolumn{4}{|c|}{ Total perched birds } & \multirow{2}{*}{$\begin{array}{l}\text { Perched birds } \\
\text { with neck } \\
\text { droop } \\
\text { (all ages } \\
\text { combined) }\end{array}$} \\
\hline & $\begin{array}{c}\text { Age } \\
\text { Unknown }\end{array}$ & Juveniles & Sub-Adults & Adults & \\
\hline \multicolumn{6}{|l|}{ White-backed vulture } \\
\hline \multicolumn{6}{|l|}{ Slender-billed vulture } \\
\hline \multicolumn{6}{|l|}{ Indian vulture (long-billed) } \\
\hline \multicolumn{6}{|l|}{ Eurasian griffon } \\
\hline \multicolumn{6}{|l|}{ Himalayan griffon } \\
\hline Egyptian vulture & \multirow{3}{*}{\multicolumn{2}{|c|}{$\begin{array}{l}\text { Total counts - no age } \\
\text { separation needed }\end{array}$}} & & & \\
\hline King vulture & & & & & \\
\hline \multicolumn{4}{|l|}{ Cinereous vulture } & & \\
\hline $\begin{array}{l}\text { Other scavengers } \\
\text { (number) }\end{array}$ & \multicolumn{5}{|c|}{$\begin{array}{l}\text { Notes and comments } \\
\text { (eg any dead birds or unusual incidents) }\end{array}$} \\
\hline Feral dog & \multicolumn{5}{|l|}{ Rats } \\
\hline Jackal & \multicolumn{5}{|c|}{ Cattle egrets } \\
\hline Crows & \multicolumn{5}{|c|}{ Steppe eagles } \\
\hline
\end{tabular}




\subsubsection{Vulture Declines Project Instructions for counting scavengers at carcasses}

This form can be used for carcass dumps or individual fresh carcasses observed at the side of the road or elsewhere. Simply record the number of fresh livestock carcasses. Please note, it is equally important to record carcasses with and without vultures. Please record any other scavengers present.

Repeat counts at carcass dumps are very useful. Please count once a month if possible. Lessfrequent counts are also very useful. It does not matter that you cannot do repeat counts of scavengers on individual livestock carcasses seen. This information is still very useful.

\section{Please record broad habitat type in the following categories:}

(a) cultivation, (b) thorn forest, (c) dry deciduous forest, (d) moist deciduous forest

(e) coastal area, (f) municipal park, (g) roadside plantation (h) other

Count the total number of all Gyps species (all species combined) perched at the carcass dump, and soaring above the carcass dump.

If there are less than 50 birds, please record species and age classes for all birds. If there are more than 50 birds, scan the birds and record species and ages and species of the first 50 birds (Gyps only) that you see when scanning the dump. If you do not have time to age birds please just separate by species and record numbers in the 'Age unknown' category. For non-Gyps species (i.e. Egyptian, King and Cinereous vulture) simply record total numbers in the 'age unknown' category.

Record numbers of birds with obvious neck droop in the neck droop column. 'Neck droop' indicates that the bird's head is hanging vertically or almost vertically towards the ground.

All ages can be combined. Again, if there are less than 50 birds record whether or not each bird has neck droop. If there are more than 50 birds, simply record whether or not each of the first 50 birds that you see when scanning the dump have neck droop.

Record any dead or very sick birds under 'Notes and comments'. Please also record rat droppings under 'Notes and comments'. Rat droppings can be recorded as none, scarce or common.

For further information please contact Dr Vibhu Prakash, Principal Scientist, Bombay Natural History Society, Hombill House, Shaheed Bhagat Singh Road, Mumbai - 4000023

Forms can be downloaded from the project website: http://www.vulturedeclines.org 


\subsection{ACAP Monitoring protocol for broad-leaved forest habitat quality}

\section{Monitoring objectives}

\section{Reasons for monitoring:}

To assure sustainable utilization of the forest resources and maintain the diversity of the broad-leaf forest species in a given area.

\section{Conservation objectives for the key feature:}

To increase or maintain the current level (2005) of broad-leaved forest species diversity and growing stock in a given forest area over the next ten years

\section{Monitoring population / area and sub-units:}

Mixed broadleaved forest in Annapurna Conservation Area- Southern Sector - Ghandruk, Lwang, Sikles, Bhujung and Southern region of Northern Sector- Lower Manang and Lower Mustang.

\section{Frequency of surveillance:}

Every 5 years

\section{Users of Results}

Primarily Project Manager and committee members- will be used in Conservation Area Management Operational Plan (CAMOP)

\section{Monitoring methods}

\section{Observation / data types:}

Forest inventory data are primarily collected as per the Inventory Guideline of Community Forestry (revised), 2004 developed by HMG/Nepal, Ministry of Forest and Soii Conservation.

\section{Data type:}

Measurement- diameter at breast height (dbh), height and canopy cover for Tree and Pole category (life form); Canopy cover will be measured by Dendrometer

Count (sapling and seedling, later for regeneration purpose)

Count: shrubs

\section{Deadwood abundance:}

Diameter at breast height (DBH) and height for dead, dying and diseased trees (3Ds) if standing,

Count (numbers) and measurement (circumference of stump) for stumps 


\section{Complete census or sample survey:}

Sample survey- firstly stratification of the area based on timber stocking (which is influenced by attributes like age class, forest type, physical terrain etc.) and secondly laying out the sample plots systematically, sampling intensity at least 0.1 (of targeted population)

General rule: Even if you don't have a prior knowledge on forest, stratification will do good, since forest as a biological entity is essentially complex.

\section{Sample area / method:}

Determining the sample area is crucial. It depends on many factors for e.g. level of precision expected, resource availability, management objective etc.

Sample area is calculated by multiplying the size of the sampling units and its number. Size of the sampling unit for different categories (life forms) as prescribed by the Inventory Guidelines, 2004, is as follows;

$25 \times 20 \mathrm{~m}^{2}(0.05 \mathrm{ha})$ - tree category $(<30 \mathrm{~cm}$ dbh, overbark)

$10 \times 10 \mathrm{~m}^{2}(0.01 \mathrm{ha})$ - pole category (10-29.9 $\mathrm{cm} \mathrm{dbh,} \mathrm{overbark)}$

$5 \times 5 \mathrm{~m}^{2}$ (0.0025 ha)- sapling (4-9.9 $\mathrm{cm}$ dbh, overbark) and regeneration $(4 \mathrm{~cm}<)$

Number of sample plot can be calculated, as the sampling intensity is known (prescribed by the guidelines).

\section{Timing of observations:}

March/April/May

\section{Potential causes of bias and rules for standardization}

Bias due to measurement

- due to sampling, unrepresentative sampling, non-response and volunteers tree

- due to Instrumental error

\section{Observer ability / training}

Not applicable

Seasonal / daily timing. Month/season should be kept constant. Return visits should be undertaken within the same 2-week period as in previous years.

\section{Sampling methods}

\section{Temporary or permanent sample location:}

Permanent plots. 


\section{Method for sample location:}

Sampling type - stratified systematic sampling in each strata

Rectangular plot (20x25 $\mathrm{m}^{2}$ for tree life form), square plot ( $10 \times 10 \mathrm{~m}^{2}$ for pole and $5 \times 5$ $\mathrm{m} 2$ for sapling and for regeneration) - nested type

While establishing the sample units in the slope, for those arms going against the slope, needs slope correction (horizontal distance $=$ slope distance $\mathrm{X} \cos \theta$ )

\section{Number of samples:}

Statistically adequate number of sample plots (thereby sample size) is required to meet the desired precision level

It also depends on various factors for e.g. condition of forest (intrinsic homogeneity). size of targeted population (ACA is quite big in area), level of precision needed (at what confidence level), however we will base the numbers on 0.1 sampling intensity as prescribed by Inventory Guidelines, 2004

\section{Monitoring requirements}

\section{Personnel responsible and time required:}

Monitoring to be coordinated by ACAP Monitoring Manager [Natural Resources Conservation Officer] with sample locations identified by applying systematic sampling techniques by field staffs (alternatively sample locations would be identified by GIS team using sample design software).

Surveys must be carried out by specifically trained ACAP Field Staff.

\section{Experience training necessary:}

Training must be given to all staff undertaking the surveys. New training (for new field staff) and refreshment (follow up) training for existing staffs at least once in five yer.r (before field works)

\section{License and access permission requirements:}

Not applicable

\section{Equipment required:}

GPS, topographic map of each forest with marked sample, photographs of key landmarks to aid relocation. Standard recording forms must be used, with copies of codes sheets (if needed). Other equipment needed are camera, pain and brush for marking, 50 meter tape, 30 meter tape, diameter tape, Abney's level or Clinometer and altimeter for altitude verification. Field safety kit.

\section{Data storage}

Monitoring manager should provide excel forms to input data. 
Original Data Recording Forms and maps should be safely stored within each Field Office and the data entered onto the standard Excel form and sent to the Monitoring Manager

Field monitoring reports must be submitted with the data. These should document the detailed methods used, including any deviations from this protocol. The reports should include: large scale maps indicating boundaries of the intensive use zone and strata (with coordinates for each zonation and strata). Transect locations with start and end coordinates; photographs of transect start-points and other important features on the transect (e.g. boulders); notes describing each transect (e.g. ridge, streamside). The reports should include the original data forms (or cross-checked typed copies) and the Codes.

\section{Data analysis}

Species and DBH class wise - density (applies to all), volume (Tree), biomass (Tree) (timber/branch/leaf)

Data are tabulated using Excel programme. Growing stock of broad leaved forest is calculated by using the formula and models as per the Inventory Guidelines.

Long term trends will be examined by correlation and regression analysis. Correlation gives the casual relation while regression provides the association.

\section{Reporting procedures:}

Every five-years on completion of survey cycle.

\section{Health and safety}

Forest work involves some inherent risks and hazards because of the places we go to and the activities we undertake. Following safety precautions applying to all field work, may minimize the risk substantially;

If at all possible, avoid going alone to the field/forest.

Wear clothing and footwear suitable for the weather, the activity and terrain

Never smoke in forests or grassland, and take care when lightening fires

Show extra care on cliffs and steep slopes

Don't incur additional risks by e.g. climbing cliffs, walking on slippery rocks, or wading

alone rivers, unless these activities are an essential part of the work

Familiarize yourself with the direction and location of the nearby village/settlement and available communication networks

Make sure you carry the First Aid and Emergency Kit 


\section{References}

Freese, F. (1984): Statistics for Land Managers, Wiley, New York

Goldsmith, F.B (1991): Monitoring for Conservation. Chapman \& Hall, London

HMG/N (2004): Guideline for Inventory of Community Forests (revised), 2004, Ministry of Forest and Soil Conservation, Kathmandu

Hurlbert, S.H (1984): Pseudoreplication and the design of ecological field experiments. Ecological Monographs 54, 187-211.

Johnson, D.H. (1999): The insignificance of statistical significance testing. Journal of Wildlife Management 63, 763-772. 


\subsubsection{ACAP Forest inventory data record sheet}

VDC

Recorded By

Date

Name of Forest

Altitude (m)

Aspect
Name of Strata

Plot No

GPS points (N/E)

Slope (degree)

$\%$ Crown cover:
Soil cover:

\section{Sign of human impacts:}

a Lopping Yes/No. If Yes (Species ..)

a Logging Yes/No, If Yes (Species (no of cut stumps)

- Non Timber Forest Products and/or Medicinal and Aromatic Plants (MAPs) collection Yes/No, If Yes (Species and extent ..)

- Grass cutting Yes/No, If Yes (Species

\section{Other impacts:}

- Grazing Yes/No, If Yes (Which animal(s)'?

- Forest fire : Yes/No

Overall condition of the forest: Regeneration / Pole / Tree

\begin{tabular}{|c|c|c|c|c|c|c|c|}
\hline \multirow{3}{*}{ S.No. } & \multirow{3}{*}{ Species } & \multicolumn{6}{|c|}{ Diameter or circumference at breast height (over bark) in $\mathrm{cm}$} \\
\hline & & \multirow{2}{*}{$\begin{array}{c}\text { Regeneration } \\
\text { (counting) } \\
\text { dbh }<4 \mathrm{~cm} \\
\text { and height }> \\
1^{\prime}\end{array}$} & \multirow{2}{*}{$\begin{array}{c}\text { Sapling } \\
\text { dbh }(4 \text { to } 9.9 \\
\mathrm{cm}) \\
\mathrm{Cr}(12.5 \text { to } 31 \\
\mathrm{cm})\end{array}$} & \multicolumn{3}{|c|}{ Pole \& Tree dbh $10 \mathrm{~cm} \&$ above } & \multirow[t]{2}{*}{ Remarks } \\
\hline & & & & $\begin{array}{l}d b h \\
(\mathrm{~cm}) \\
\text { or } \mathrm{Crf}\end{array}$ & $\begin{array}{l}\text { Tree top I } \\
\text { bottom } \\
\text { angle }\end{array}$ & \begin{tabular}{|c|} 
Distance \\
of the \\
tree from \\
Obs.
\end{tabular} & \\
\hline \multicolumn{8}{|c|}{ 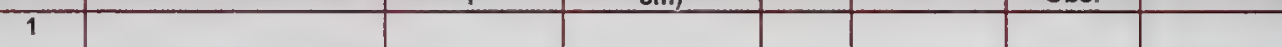 } \\
\hline \multicolumn{8}{|l|}{2} \\
\hline \multicolumn{8}{|l|}{3} \\
\hline \multicolumn{8}{|l|}{4} \\
\hline \multicolumn{8}{|l|}{5} \\
\hline \multicolumn{8}{|l|}{6} \\
\hline \multicolumn{8}{|l|}{7} \\
\hline \multicolumn{8}{|l|}{8} \\
\hline \multicolumn{8}{|l|}{9} \\
\hline \multicolumn{8}{|l|}{10} \\
\hline \multicolumn{8}{|l|}{11} \\
\hline \multicolumn{8}{|l|}{12} \\
\hline \multicolumn{8}{|l|}{13} \\
\hline \multicolumn{8}{|l|}{14} \\
\hline \multicolumn{8}{|l|}{15} \\
\hline \multicolumn{8}{|l|}{16} \\
\hline \multicolumn{8}{|l|}{17} \\
\hline \multicolumn{8}{|l|}{18} \\
\hline \multicolumn{8}{|l|}{19} \\
\hline \multicolumn{8}{|l|}{20} \\
\hline \multicolumn{8}{|l|}{21} \\
\hline 22 & & & & & & & \\
\hline
\end{tabular}

Note: $\mathrm{Crf}=$ Circumference at breast height, Obs $=$ Observer 


\section{Evidence of wildlife}

\begin{tabular}{|c|c|c|c|}
\hline S. No. & Species & Number of animals & Type of sighting \\
\hline 1 & & & \\
\hline 2 & & & \\
\hline 3 & & & \\
\hline 4 & & & \\
\hline 5 & & & \\
\hline 6 & & & \\
\hline 7 & & & \\
\hline 8 & & & \\
\hline 9 & & & \\
\hline 10 & & & \\
\hline
\end{tabular}

List the name of NTFP and MAP found within the plot

Other observations/comments of the surveyor 


\subsection{ACAP Monitoring protocol for broad-leaved forest birds \\ Monitoring objectives}

\section{Reasons for monitoring:}

- Forest birds are of conservation importance, and include some threatened or regionally important species.

- Some forest birds are indicators of high forest quality or ecological value.

\section{Conservation objectives for the key feature:}

To maintain or increase the current levels of breeding bird species richness and population size in selected forest indicator species over the next 10 years, to 2015.

\section{Monitoring population / area and sub-units:}

Mixed broadleaved forest in Annapurna Southern Sector - Ghandruk, Sikles, Lower Mustang.

Mixed broadleaved forest in Manang.

\section{Frequency of surveillance:}

Annual

\section{Monitoring methods}

\section{Observation / data types:}

Direct visual observations and calls/songs of birds, mostly ascribed to species.

Bird species richness (species detected in each sample plot)

Relative bird abundance from Timed Species Count (TSC)

Frequency of species occurrences per 10 -minute observation period

Abundance estimates from point counts

\section{Complete census or sample survey:}

Two-stage sample

\section{Sample area / method:}

TSC methods over three 60 minutes counts over fixed routes. Route to be deduced beforehand from reconnaissance and previous habitat monitoring on 1 st visit (see Forest Habitat Quality Protocol).

Observers should walk slowly and quietly along the preset and mapped route, recording all birds seen or heard within the survey square on the standard survey form (see below). 
Observers should stop walking and listen quietly for about 3 minutes, approximately midway through each 10 minute period. Birds seen outside the square may be recorded in the appropriate column on the survey form.

\section{Timing of observations:}

March/April, from 1 hour after sunrise for 4 hours (c. 7 am - 11 am).

\section{Potential causes of bias and rules for standardization:}

Observer ability i training. Surveyor teams should include at least one fully experienced and trained ornithologist (see below).

Seasonal / daily timing. This should be kept constant. Return visits should be undertaken within the same 2-week period as in previous years.

Weather. Surveys should not be undertaken during heavy rain or snow, or in high winds (c. $>15 \mathrm{mph}$ ).

Habitat change. This cannot be controlled for. Therefore care should be taken in interpreting changes where habitat changes may have affected visibility.

\section{Sampling methods}

\section{Temporary or permanent sample location:}

Permanent primary and secondary routes.

\section{Method for sample location:}

Stratified random location of primary samples $(2 \mathrm{~km} \times 2 \mathrm{~km})$, stratification by altitude / habitat type [to be carried out by ACAP]. Exclude areas outside mixed-broadleaved zone, and exclude steep ground to produce a sample population area.

Secondary routes to be spread across plot to encompass all variation in forest habitats types within the survey plot, including different age classes, densities, habitat type and degrees of degradation. Minor paths may be used if they do not interfere with tree cover. But large tracks where cover is broken such that edge species dominate must NOT be used. Treeless shrubland and grassland etc should not be included.

The route should be mapped as accurately as possible with distinctive features (e.g. an obvious type of large tree) noted and coordinates recorded using a GPS (where tree cover allows).

\section{Number of samples:}

To be allocated depending on resources; minimum of 10 per key monitoring area (or 5 per stratum). 


\section{Monitoring requirements}

\section{Personnel responsible and time required:}

Teams of two ornithologists. One or two mornings fieldwork per primary plot, depending on terrain (i.e. a total of about 4 hours). Remaining time each day to be used for traveling to vicinity of the next sample.

\section{Experience training necessary:}

At least one of the two ornithologists to be fully experienced with forest bird identification and trained in method. The other ornithologist may be a trainee.

\section{Licence and access permission requirements:}

Not applicable

\section{Equipment required:}

Binoculars (8x or 10x magnification), map with marked sample areas and coordinates, compass, GPS, field notebook, standard recording forms (see below), watch with stopwatch facility, and field first aid / safety kit.

\section{Data storage}

Original Data Recording Forms should be safely stored within each Field Office and the data entered onto the standard Excel summary form and sent to the Monitoring Manager [ACAP to devise based on the recording forms].

\section{Data analysis}

\section{Reporting procedures:}

Annually 


\subsubsection{ACAP forest bird survey recording form}

\section{Part a: count details}

\section{Date (day/month/year):}

\section{Counter details}

Name of lead counter:

Address:

Other observers / trainees present:

\section{Count location}

ACAP Unit Conservation Area:

Nearest town / village:

Primary plot number:

Primary plot coordinates: $\quad$ SE corner: NW corner:
Altitude - $\min (\mathrm{m})$ :
Altitude - max:
Aspect:

Broad habitat types: $\%$ cover within survey plot:
a) Cultivated:
e) Montane deciduous forest / shrubland:
b) Broad-leaved forest:
f) Grassland:
c) Mixed broad-leaved / coniferous forest:
g) Barren soil / rock:
d) Coniferous forest:
i) Other:

\section{Visit details}

Start time:

End time:

Weather conditions:

Temperature (circle): Cold $\left(<5^{\circ} \mathrm{C}\right) / \mathrm{Cool}\left(5-10^{\circ} \mathrm{C}\right) / \mathrm{Mild}\left(10-15^{\prime \prime} \mathrm{C}\right) / \mathrm{Warm}\left(15-20^{\prime \prime} \mathrm{C}\right)$ $/$ Hot $\left(>20^{\circ} \mathrm{C}\right)$

Cloud cover (estimate to nearest $10 \%$ ):

Wind direction: Wind speed: still / light / breeze / strong breeze / near gale / gale

\section{Comments}


Part b: Observations

(page ... of ...)

\begin{tabular}{|l|l|l|}
\hline Lead observer & Primary plot no. & Transect number \\
\hline & & \\
\hline
\end{tabular}

\begin{tabular}{|l|l|l|}
\hline Start & Coordinates & Coordinates \\
\hline & & \\
\hline Finish & Coordinates & Coordinates \\
\hline & & \\
\hline
\end{tabular}

\begin{tabular}{|l|l|l|}
\hline Date & Time started & Time finished \\
\hline & & \\
\hline
\end{tabular}

\begin{tabular}{|c|c|c|c|c|c|c|c|c|c|}
\hline & \multicolumn{6}{|c|}{ Time period seen in } & & \multicolumn{2}{|c|}{ Analysis } \\
\hline Species & $0-10$ & $10-20$ & $20-30$ & $30-40$ & $40-50$ & $50-60$ & Out & Score & Freq \\
\hline & & & & & & & & & \\
\hline & & & & & & & & & \\
\hline & & & & & & & & & \\
\hline & & & & & & & & & \\
\hline & & & & & & & & & \\
\hline & & & & & & & & & \\
\hline & & & & & & & & & \\
\hline & & & & & & & & & \\
\hline & & & & & & & & & \\
\hline & & & & & & & & & \\
\hline & & & & & & & & & \\
\hline & & & & & & & & & \\
\hline & & & & & & & & & \\
\hline & & & & & & & & & \\
\hline & & & & & & & & & \\
\hline & & & & & & & & & \\
\hline & & & & & & & & & \\
\hline & & & & & & & & & \\
\hline & & & & & & & & & \\
\hline & & & & & & & & & \\
\hline & & & & & & & & & \\
\hline & & & & & & & & & \\
\hline & & & & & & & & & \\
\hline & & & & & & & & & \\
\hline & & & & & & & & & \\
\hline
\end{tabular}

\begin{tabular}{|l|l|l|}
\hline 1 & Coordinates & Coordinates \\
\hline 2 & Coordinates & \\
\hline & & Coordinates \\
\hline
\end{tabular}




\subsection{ACAP Monitoring protocol for remote sensing of habitat extent and quality}

This monitoring protocol is designed to assess, at the ACA level, the quality of habitats using remote sensing data as indicated by spatio-temporal changes in habitat extent and quality. It will also assess fragmentation characteristics of the habitat patches from a landscape level perspective.

\section{Monitoring objectives}

\section{Reasons for monitoring:}

- A general idea of the trend of biodiversity in a habitat scale can be derived from monitoring of habitat extent and location as it directly affects the distribution and abundance of floral and faunal diversity

- Habitat extent and quality is a direct measure of biodiversity

- Spatio-temporal changes in habitat types and their extent is necessary to access the effectiveness of management interventions

- Data on habitat fragmentation is useful to plan, monitor and evaluate habitat and species conservation.

\section{Conservation objectives for the key feature:}

- To generate information on coverage, spatio-temporal changes and fragmentation characteristics of habitats of ACA region for use by ACAP management team to plan. monitor and evaluate the habitat and species conservation activities.

\section{Users of monitoring results:}

- ACAP management planning team (includes KMTNC program and monitoring unit, ACAP team and Conservation Area Management Committees)

\section{Monitoring population I area and sub-units:}

- Whole ACA region

\section{Frequency of surveillance:}

- Every 5 years

\section{Monitoring methods}

\section{Observation / data types:}

- ASTER (Advanced Spaceborne Thermal Emission and Reflection) data set for 2000 onwards (This data set is available free of charge via http:// asterweb.jpl.nasa.gov/) and has spatial resolution of $15 \mathrm{~m} \times 15 \mathrm{~m}$ ).

- Landsat data for years before 2000 (http://landsat.gsfc.nasa.gov/)

- Digital elevation model - it will be generated from the clevation contour data of HMG/N (2002) 
- Ground truth data (collected using GPS set); complemented by:

- data generated from broad-leaved forest habitat quality monitoring and

- reports of KMTNC/ACAP Natural Resources Conservation Program related to forest inventory

\section{Complete census or sample survey:}

Sample ground truth survey (from representative habitat types all over ACA)

\section{Sample area / method:}

Unsupervised classification of satellite image will be done - resulting classes will be the strata for ground truth data collection. To ensure better representation, unsupervised classification will be done to get 3 times classes of the habitat types. Ground truth data will be collected from all over the ACA using stratified random sampling.

\section{Timing of observations:}

Satellite image data of June-August has to be acquired because most of the vegetation is detectable at this time.

\section{Potential causes of bias and rules for standardization:}

For time series analysis, satellite image data have to be of the same season as season has impact on the reflectance value of vegetation. While using data from multiple sensors, care should be taken of their spatial resolution. Different classification methods and softwares might also affect results.

\section{Sampling methods}

\section{Temporary or permanent sample location:}

Temporary

\section{Method for sample location:}

Stratified (as per the result of unsupervised classification) random sampling. Good interspersion and independence of samples is required.

\section{Number of samples:}

At least 10 from each strata of every Unit Conservation Offic

\section{Monitoring requirements}

\section{Personnel responsible and time required:}

- GIS Officer for co-ordination, data storage and analysis - 6 months

(18. Concerned UCO Monitoring Co-ordinator for field data collection - 2 weeks

( ACAP Monitoring Co-ordinator for overall co-ordination - 2 weeks

2 Natural Resource Conservation Assistants (NRCAs) of concerned UCOs ipproximaty 15 man months for collecting ground truth data from all over the ACA 


\section{Experience training necessary:}

- GIS Officer - Knowledge of satellite image interpretation in Erdas Imagine remote sensing software, theoretical knowledge of satellite image interpretation in digital environment, and Knowledge of Fragstats software and landscape indices

- NRCAs - GPS operation and data recording, locating ground truth points on the ground

\section{License and access permission requirements:}

None

\section{Equipments required and cost:}

a Computer (preferably Pentium IV) with minimum of 512 MB RAM, 80 GB hard drive with a mirror drive for backup - 1 set (Approx. NRs. 120,000)

- Erdas Imagine remote sensing software (www.erdas.com) - 1 license (Approx. NRs. 800,000 on discounted price)

- GPS set - 2 in each UCO (14 total) (Approx. NRs. 33,000/set)

- Fragstats (version 3.0) software - 1 set (Free) (http:/www.umass.edu/landeco/ research/fragstats/fragstats.html)

- ArcView or ArcGIS software (for maps layout and production) (www.esri.com) - 1 license (Approx. NRs. 500,000 for ArcGIS with Spatial Analyst)

- Colour printer (for final maps production) preferably plotter of $\mathrm{A} 0$ size (to print in large size to show more details) - 1 set (A4 size printer - NRs. 5,000 to 300,000 depending on quality; Plotter - Approx. NRs. 800,000 to 2,000,000 depending on size and quality)

\section{Data storage}

All the satellite data will be stored in the GIS lab computer at ACAP HQ under the designated software domain. Ground truth and other inventory data will be primarily stored in MS-Excel format. Proper back up of the data has to be done in digital and hard copy (printed) format also.

\section{Data analysis}

\section{Data will be analyzed to get:}

- Habitat classes (Refer to Annex I) using Supervised Classification method (Richards, 1999 and Lillesand et.al. 2004 will be used as reference for detailed procedures and quality insurance). Digital elevation data might help improve the classification result (Shrestha and Zinck, 2001). Comparison of time series habitat classes will give spatio-temporal changes in the habitat types and quality.

- Landscape indices (McGarigal and Marks, 1994; Rutledge, 2003) - for accounting fragmentation (Southworth et. al., 2002). 


\section{Reporting procedures:}

Every 5 years to the senior management (within 3 months of completion of ground truth data collection). Report will include the result as maps, tables and graphs to show status and changes in habitat extent, quality and fragmentation. Powerpoint presentations and webpages will also be part of the report. Posters and booklet will be produced in Nepali language to report the results to the local communities. Seminars will be held at local (at least UCO level) and national level to disseminate the findings.

\section{References}

KMTNC/ACAP/BCDP (1994): Final Draft Report. King Mahendra Trust for Nature Conservation, Annapurna Conservation Area Project, Biodiversity Conservation Data Project. March 1994.

His Majesty's Government of Nepal (2002): Digital Topographic Base Maps in ArcInfo format - based on 1992/1996 aerial photographs (field verification done in various years - 2000 for ACA region) - 1:50,000 for mountains and 1:25,000 for Terai. HMG/N, Topographic Survey Branch, Min Bhawan, Kathmandu.

Lillesand, T.M., Kiefer, R.W. and Chipman, J.W. (2004): Remote Sensing and Image Interpretation. Fifth Edition. John Wiley \& Sons, Inc. ISBN: 0-471-15227-7.

McGarigal, K. \& Marks, B.J. (1994): Fragstats: spatial pattern analysis program for quantifying landscape structure, v. 2.0. Corvallis, OR, Oregon Forest Science Lab, Oregon State University. p. 134.

Richards, J.A. (1999): Remote Sensing Digital Image Analysis. Springer-Verlag, Berlin. p. 240.

Rutledge, D. (2003): Landscape indices as measures of the effects of fragmentation: can pattern reflect process? DOC Science Internal Series 98. New Zealand Department of Conservation. ISBN 0-478-22380-3.

Shrestha, D.P. and Zinck J.A. (2001): Landuse Classification in Mountainous Areas: Integration of Image Processing, Digital Elevation Data and Field Knowledge (application to Nepal). JAG, 3 (1), pp 78-85.

Southworth J. Nagendra H. \& Tucker C. (2002): Fragmentation of a Landscape: incorporating landscape metrics into satellite analyses of land-cover change. Landscape Research, Vol. 27, No. 3, pp 253-269. 


\subsubsection{Habitat Classes}

SN Habitat Type

\section{A. Sub-tropical Zone}

A.1 Broad leaved forests (Includes sub-types Hill Sal forests, sub-tropical deciduous hill forests, semi-evergreen forests and Schima-Castanopsis forests)

A.2 Shrublands

A.3 Grasslands

B. Temperate Zone

B.1 Broadleaved forests (Most of the patches dominated either by Quercus species or by Rhododendron)

B.2 Conifer forests

B.3 Shrublands

B.4 Grasslands

C. Sub-alpine Zone

C.1 Broadleaved forests (Dominated in some places by Rhododendron and in some places by Betula with fir, spruce and scrub)

C.2 Conifer forests (Pine trees associated with Taxus, Tsuga and Betula)

C.3 Shrublands

C.4 Grasslands (numerous herbs and forbs dominated by Carex spp)

D. Alpine Zone

D.1 Alpine scrub and grassland (It consists of moist and dry alpine scrub and grassland)

\section{E. Special Habitats}

E.1 Agricultural fields and settlements

E.2 Rivers and Ponds

E.3 Abandoned agricultural land

E.4 Bareland

E.5 Permanent snow cover 



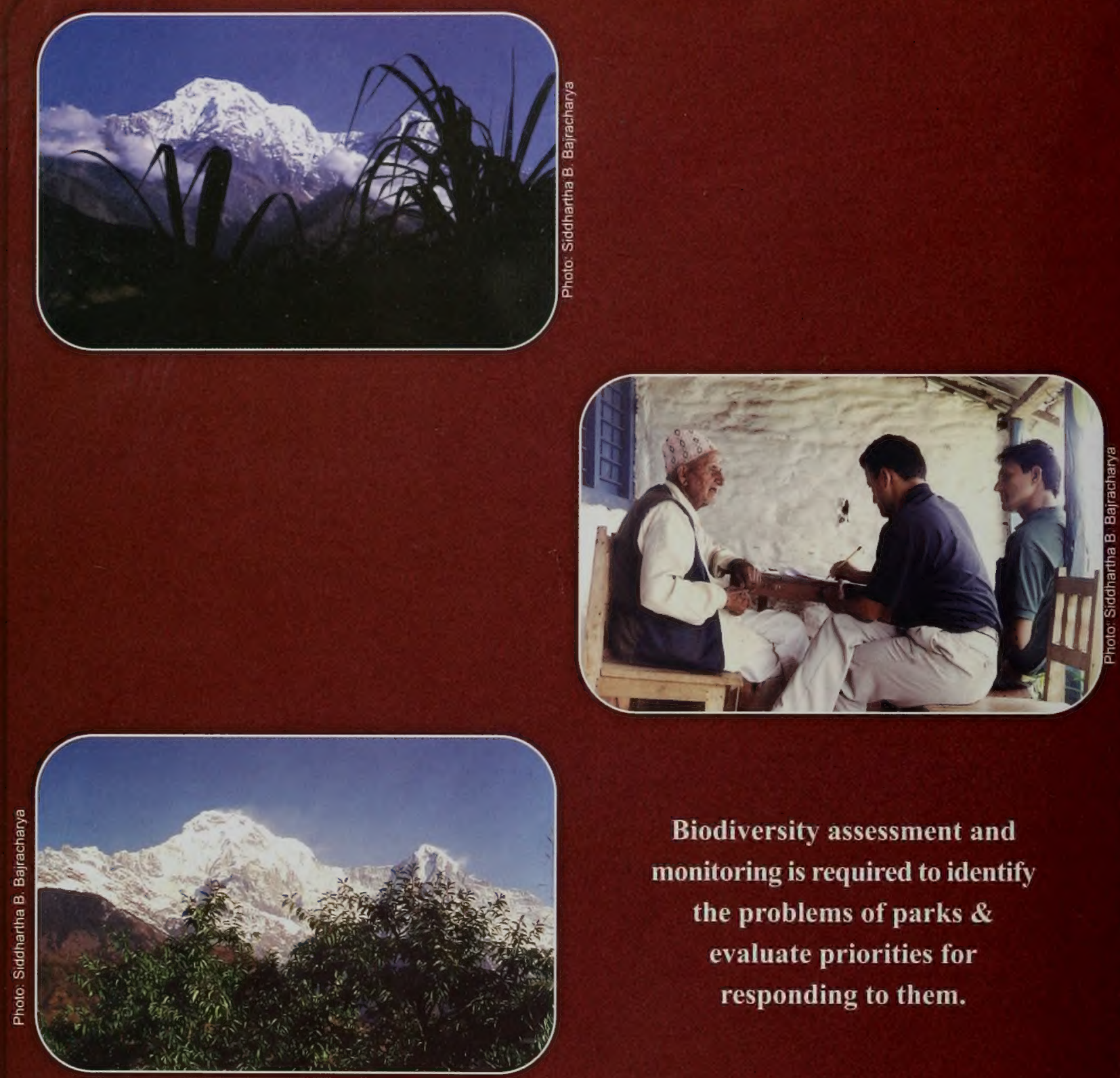

Biodiversity assessment and monitoring is required to identify the problems of parks \& evaluate priorities for responding to them. 